

\title{
LEGML DISCLAIUER
}

This reporti was p repared as an account of work sponsored by An agency of the Uniled States Government, Neither the United States Governifient nor any edency thereof nor any of their employ aes, no any of thel contractors subcontrabiors

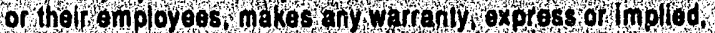
or assumes any legal tiability or responsibility or the accuracy completeness or any thild party's 486 or the results of such use ol any information ap paralus, product, of process disclosed or represents that lis use would not hintinge privalaly owned righí. Heterence herein to any spocific commercial product orocess or service by trade name. trademark, manufacurer or olhervise, does not necossarily consitute or im ply tis endorsement, recommendation, or Tavoring by the United stalo covernment or any agency there of of te contractors ord ubcontractors. Tha viewe and oplinions of aúthors expressed herein do not hecessarily stale of reflect those of the Unifed States Government or any agency thereof:

This report has been reproduced from the best avallable copy. Available in paper copy and microfiche.

\author{
Available to the U:S Dopartment of Energy \\ and its contraciors from \\ Office of Scientilic and Tochnical Information \\ P. $0.80 \times 62$ \\ Oak Ridge, TN 37831 \\ (615) $576-8401$
}

Available to the puslic from the U.S. Department of Commerce Nationál Technical Inlormation Service

5285 Port Royal Road

Springlield, VA 22161

(703) 487.4650

Pinitod in tho Uniled Statos of Amorica

DISCLM-1.CHP (1.91) 


\section{Tank Farm Surveillance and Waste Status Summary Report for January 1993}

B. M. Hanlon

Date Fublished

March 1993

Prepared for the U.S. Department of Energy Office of Environmental Restoration and Waste Management

\section{(W) Westinghouse P.O. Box 1970}


WHC-EP-0182-58

\section{APPROVAL}

Prepared by: $\frac{\text { B.M. Atanlow }}{\substack{\text { B.M. Hanion } \\ \text { Engineer }}} \frac{2-25-9,3}{\text { Date }}$

Approved by: $\frac{2 \sum(2 \text { aymond }}{\substack{\text { R. E. Raymond } \\ \text { Manager, Single-Shell } \\ \text { Tank Technology }}} \frac{3 / 11 / 93}{\text { Date }}$ 


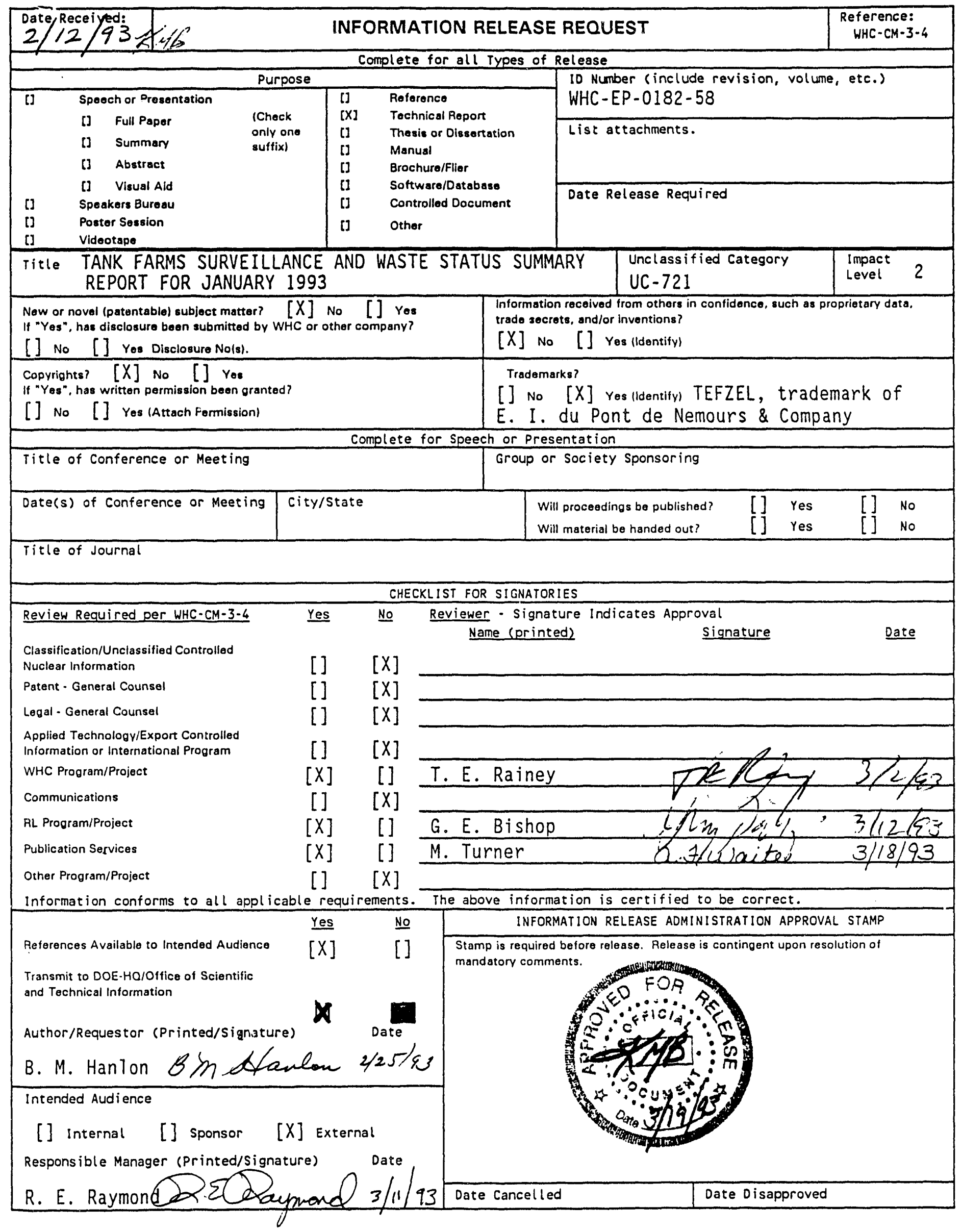


WHC-EP-0182-58

\title{
TANK FARM SURVEILLANCE AND WASTE STATUS \\ SUMMARY REPORT FOR JANUARY 1993
}

B. M. Hanion

\begin{abstract}
This report is the official inventory for radioactive waste stored in underground tanks in the 200 Areas at the Hanford Site. Data that depict the status of stored radioactive waste and tank vessel integrity are contained within the report. This report provides data on each of the existing 177 large underground waste storage tanks and 49 smaller catch tanks and special surveillance facilities, and supplemental information regarding tank surveillance anomalies and ongoing investigations. This report is intended to meet the requirement of U.S. Department of Energy-Richland Operations Office Order 5820.2A, Chapter I, Section 3.e. (3) (DOE-RL, 1990, Radioactive Waste Management, U. S. Department of Energy-Richland Operation Office, Richland, Washington) requiring the reporting of waste inventories and space utilization for Hanford Tank Farm Tanks.
\end{abstract}


WHC-EP-0182-58

This page intentionally left blank. 
SUMMARY . . . . . . . . . . . . . . . . . . . 1

TANK STATUS

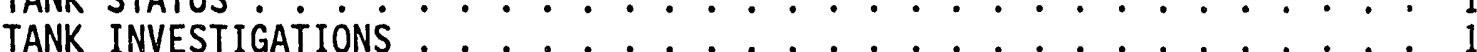

HIGHLIGHTS ........................... 3

Appendixes:

A. TANK AND EQUIPMENT CODE AND STATUS DEFINITIONS . . . . . . . . . A-1

Tank and Equipment Code/Status Definitions . . . . . . . . . . A-3

B. TANK FARM CONFIGURATION, STATUS AND FACILITY CHARTS . . . . . . . . . B-1

1 High-Level Waste Tank Configuration . . . . . . . . . . . . . . B-3

2 Double-Shell Tank Instrumentation Configuration . . . . . . . B-4

3 Single-Shell Tank Instrumentation Configuration . . . . . . . B-5

4 Double-Shel1 Tank Status . . . . . . . . . . . . . B-6

5200 E Single-Shell Tank Status ............. . . B-7

6200 W Single-Shell Tank Status ... . . . . . . . . . . . B-9

7 Tank Farm Facilities Quick Reference . . . . . . . . . . . . B-11

8 Hanford Tank Farms Facilities Chart ............ B-12

c. MONTHLY SUMMARY ........................ . . -1

1 Monthly Summary . . . . . . . . . . . . . . . C-3

2 Tank Use Summary . . . . . . . . . . . . . . . . . . . C C 4

3 Inventory Summary by Tank Farm . . . . . . . . . . . . . . C-5

4 Inventory and Status by Tank - Double-Shell Tanks . . . . . . . C-6

5 Inventory and Status by Tank - Single-Shell Tanks . . . . . . . C-9

D. PERfORMANCE SUMMARY ..................... . D-1

1 Performance Summary . . . . . . . . . . . . . D-3

E. LIQUID STATUS AND PUMPABLE LIQUID REMAINING IN TANKS . . . . . . . . . E-I

1 Liquid Status and Pumpable Liquid Remaining in Tanks . . . . . . . E-3

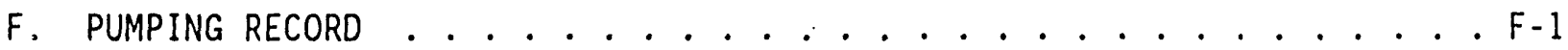

1 Pumping Record . . . . . . . . . . . . . . . . . F-3

G. CATCH TANKS AND SPECIAL SURVEILLANCE FACILITIES . . . . . . . . . . . . G-1

1 East and West Area Catch Tanks and Special

Surveillance Facilities (Active) . . . . . . . . . . . . . G-3

2 East Area Catch Tanks and Special Surveiliance

Facilities (Inactive) .. . . . . . . . . . . . . . . G-4

3 West Area Catch Tanks and Special Surveillance

Facilities (Inactive) .............. . G-5

H. LEAK VOLUME ESTIMATES ..................... H-1

1 Single-Shell Tank Leak Volume Estimates . . . . . . . . . . H-3 
WHC -EP-0182-58

This page intentionally left blank. 


\section{LIST OF FIGURES}

1 Current Status and Contingency Space for the 242-A

Evaporator Restart ...................... 21

2 Master Core Sampling Schedule . . . . . . . . . . . . . . . 22

3 Waste Volumes for Sound Single-Sheil Tanks . . . . . . . . . . . . . . 23

4 Waste Volumes for Assumed Leaking Single-Shell Tanks . . . . . . . . 24

\section{LIST OF TABLES}

1 Watch List Tanks . . . . . . . . . . . . . . . . . . . 7

2 Tanks Containing $>1,000$ gram mole of Ferrocyanide (Watch List Tanks) . . . . . . . . . . . . . . . . . 8

3 Tanks with Potential for Hydrogen or Flammable Gas Accumulation Above the Flammability Limit (Watch List Tanks) . . . . . . . . . 10

4 Tanks Containing Concentrations of Organic Salts $>3 \%$ Weight TOC (Watch List Tanks) . . . . . . . . . . . . . . . 11

5 Single-Shell Tanks with High Heat Loads $(>40,000 \mathrm{Btu} / \mathrm{hr})$. . . . . . 12

6 Double-Shell Tank Waste Type and Space Allocation . . . . . . . . . 14

7 Automatic Food Instrument Company (FIC) Gauges Out of Service . . . 15

8 Single-Shell Tanks Monitoring Compliance Status . . . . . . . . . . 16

9 Double-Shell Tanks Monitoring Compliance Status . . . . . . . . . . 20

\begin{tabular}{|l|l|l|}
\hline \multicolumn{3}{|c|}{ METRIC CONVERSION CHART } \\
\hline 1 inch & $=$ & 2.54 centimeters \\
\hline 1 foot & $=30.48$ centimeters \\
\hline 1 gallon & $=$ & 3.80 liters \\
\hline 1 ton & $=0.90$ metric tons \\
\hline${ }^{\circ} \mathrm{F}=\left(\frac{9}{5}{ }^{\circ} \mathrm{C}\right)+32$ \\
\hline \\
1 Btu/h $=2.930711$ E-01 watts \\
(International Table)
\end{tabular}


WHC-EP-0182-58

This page intentionally left blank 
WHC-EP-0182-58

TANK FARM SURVEILLANCE AND WASTE STATUS SUMMARY

REPORT FOR JANUARY 1993

SUMMARY

Note: Changes from the previous month are in bold print.

TANK STATUS

\begin{tabular}{|c|c|c|}
\hline Category & Quantity & Date of Last Change \\
\hline In-Service Tanks ${ }^{c}$ & 28 double-shell & $10 / 86$ \\
\hline Out-of-Service Tanks ${ }^{a}$ & 149 single-shell & $07 / 88$ \\
\hline Assumed Leaker Tanks ${ }^{f}$ & 67 single-shell & $10 / 92$ \\
\hline Sound Tanks & $\begin{array}{l}28 \text { double-shel1 } \\
82 \text { single-shell }\end{array}$ & $\begin{array}{c}1986 \\
10 / 92 \\
\end{array}$ \\
\hline Interim Stabilized Tanks ${ }^{b, d}$ & 105 single-shel1 & $09 / 90$ \\
\hline Not Interim Stabilized ${ }^{f}$ & 44 single-shell & $09 / 90$ \\
\hline Interim Isolated Tanks ${ }^{e}$ & 98 single-shell & $09 / 91$ \\
\hline
\end{tabular}

a All 149 single-shell tanks were removed from service (i.e., no longer authorized to receive waste) as of November 21, 1980.

of the 105 tanks classified as interim stabilized, 58 are listed as assumed leakers. The total of 105 interim stabilized tanks includes eight tanks that do not meat current established supernatant and interstitial liquid stabilization criteria: 104-B, 107-B, 110-B, 111-B, 110-BX, 102-T, 112-T, and $110-U$.

c Six double-shell tanks listed as "in service" are currently included on the Hydrogen Watch List and are thus prohibited from receiving waste in accordance with "Safety Measures for Waste Tanks at Hanford Nuclear Reservation," Section 3137 of the National Defense Authorization Act for Fiscal Year 1991, November 5, 1990, Public Law 101-510. Stabilized.

Of the 48 single-shell tanks on Watch Lists, 21 have been Interim I solated.

ef the 48 single-shell tanks on Watch Lists, 20 have been Interim

Nine of these tanks are both assumed leakers and not interim stabilized. See Appendix $H$ for more details.

\section{TANK INVESTIGATIONS}

Tank 241-SY-101. The surface level within this tank continues to fluctuate. The surface level increase/decrease phenomena has been observed since 1981, and is attributed to the slow buildup and relatively fast release of gas within the waste. An investigation into solutions to this slurry growth problem is ongoing. Multiple Event Fact Sheets, a Critique Report, Occurrence Reports, Discrepancy Reports, and Unusual Occurrence Reports have been issued. The Food Instrument Company (FIC) surface level monitor showed an increase from 408.80 inches on January 1 to 411.20 inches by January 31,1993 . The 
manual tape increased from 409.25 to 414.75 inches by January 31,1993 . The radar gauge reading was 410.86 inches January 1 and 413.08 inches on January 31, 1993. The surface level measuremants for the radar gauge, FIC, and manual tape are different due to the location of the measurement devices, irregular surface, and the different method by which the level is obtained.

Tank 241-SX-105. An Interstitial Liquid Level (ILL) baseline of 21.2 feet was established for Tank 105-SX Liquid Observation Well (LOW) on August 27, 1986. The current scan of January 22, 1993, shows a 0.2-foot decrease below the reference baseline. The decrease criteria is -0.3 foot. LOW monitoring has been increased from quarterly to every two weeks. In-tank photographs taken June 15, 1988, show a dry cracked, uneven surface. The FIC is in the intrusion mode of operation, and the FIC plummet is suspended over solids. Drywell scan data remain stable. Work is in progress to repair the radiation detection laterals underneath this tank to provide improved leak detection. The Tank Leak Assessment Review Board has recommended waiting for the lateral scans and evaporation analysis, currently in progress, to be completed orior to deciding on the tank integrity. Estimated completion for the evapor ition analysis and lateral restoration is March 1993.

Tank 241-5X-106. The surface level measurement baseline was aifjusted on April 3, 1991, for an increase attributed to a steam coil leak that occurred March 13, 1991 (reference Occurrence Report WHC-91-0206-TFARM). The level increased an additional 1.20 inches by May 17, 1991, after baseline change. There has been a decreasing trend in the surface level measurement from 209.90 inches on May 17, 1991, to 206.00 inches by December 31, 1992. The decrease criteria is 5.00 inches from reference baseline of 208.70 inches. The FIC plummet is contacting solids. In-tank photographs taken June 1, 1989, show what appears to be a floating solid (foam-like) surface. The LOW ILL increased from 16.3 feet (from reference baseline) to 16.6 feet by March 19 , 1991 , and remained at 16.6 feet to 0ctober 24, 1991. The ILL decreased to 16.3 feet $(-0.3$ feet) by October 8,1992 , and has remained at 16.3 feet during January 1993. An evaporation study has been completed by Waste

Characterization Analysis. The analysis indicated that observed liquid level decreases could be attributed to evaporation and are within the range expected from evaporation. A small leak could easily be masked by modeling uncertainties. Low monitoring has been increased to very two weeks. Drywells associated with 106-SX are stable. The technical evaluation of the alert condition in this tank is in progress by Engineering. The expected completion date for the results is February 16, 1993.

\section{Potential or Assumed Leaks:}

Tank 241-T-101. A surface level decrease of 2:60 inches from the previously established liquid level of 44.20 inches was noted on September 18, 1992, following maintenance on the 101-T Level Indicator (FIC). Because the FIC had been operating only sporadically since December 1991, and no reliable surface level data was obtained between December 1991 and July 1992, the decrease was assumed to be due to the faulty FIC, and no occurrence reporting action was taken during that time. Occurrence Report RL-WHC-TANKFARM-1992-0073 was issued October 1, 1992, and Tank 101-T was declared an assumed leaker on october 4, 1992. The FIC surface level measurement was 41.50 inches on October 31,1992 , and increased to 43.80 inches after a reference elevation check was performed by instrument technicians on November 14, 1992. The FIC surface level measurenient is reading 43.55 inches on January 31,1993 . A manual tape surface level measurement device was installed on December 18, 
1992. The measurement from this device is 40.25 inches as of January 31 , 1993. Drywell scan data was reviewed and is stable. Drywell monitoring (gross gamma) frequencies have been increased to weekly. Plans are under.vay to begin pumping this tank by March 15, 1993. The photography work package is in place, but will not be scheduled until after Tank 101-SY Window " $H$ " work is complete. Estimated completion date for vapor space sampling is February 15, 1993.

\section{Potential or Assumed Intrusions:}

The following tanks have been reported for potential in-leakage (intrusions) from known/unknown sources.

Tank 241-B-202. A steady increase in the surface level measurement has been observed since December 1984. The manual tape pencil plummet is contacting liquid. When the quarterly reading was obtained on October 6,1992 , the level was recorded as 144.75 inches, thus exceeding the 2.00-inch increase criteria from the established baseline of 142.50 inches The surface level measurement was rechecked on October 9, 1992, (145.50 inches) and October 13 (145.00 inches), verifying the increase and that the criteria had been exceeded. The monitoring frequency has been increased from quarterly to monthly. The reading for January 31 , 1993, was 145.00 inches. The technical evaluation of the alert condition in this tank is in progress by Engineering. The expected completion date for the results is February 16, 1993. Tank 202-B has been added to the Alert List and will be reported in accordance with applicable reporting requirements.

Tank 241-S-107. A slow increase in the surface level has been observed since May 1987. The surface level measurement increased 1.10 inches in September 1991, when water was added to the tank in order to install a saltwell screen. The reference baseline was adjusted to reflect this water addition. On August 3 , 1992, the surface level measurement was 144.50 inches, thus exceeding the 2.00 -inch increase criteria from the established baseline of 142.40 inches. The FIC was reported out of service on August 17, 1992. After the FIC was repaired on September 21 , 1992, the surface level measurement was 144.20 inches, which is within criteria limits. The reading level for January 26 , 1993, was 144.25 inches. This tank will remain under close surveillance for additional unexplained surface level increases.

Tank 241-TX-115. The LOW scans revealed an ILL increase over baseline in excess of the established 0.4-foot increase criteria in May 1987. Comparison of past and present in-tank photographs show no significant change in surface conditions or obvious evidence of intrusion. Environmental Protection Deviation Report \#88-08 was issued on May 18, 1988. Event Fact Sheet \#TF-EFS80-140 was issued January 9, 1990. The ILL showed an additional increase of 0.4 feet on March 1, 1991. The increase was verified on March 11, 1991. These LOWs are monitored quarterly, alternating every six weeks with the neutron and gamma probes. No further increase of the ILL has been observed since March 1, 1991. The LOW was last scanned with the neutron probe on October 23, 1992.

Tank 241-TY-102. On November 8, 1992, the automatic FIC surface level measurement of 32.10 inches exceeded the 0.50 -inch increase criteria from the reference baseline of 31.40 inches. Discrepancy Report S\&DA-92-489 was issued November 9, 1992. The tank has a history of icicle buildup on the FIC piummet 
and intrusions. A request was made on November 16 for Tank Farm Operations to flush the FIC plummet. Work Order 2W-92-01155 was written to perform the flush, scheduled for December 3, 1992. The reference elevation of the FIC was verified on December 17, 1992. The FIC plummet was clean.

\section{HIGHLIGHTS}

\section{Criticality Safety Issues}

On Apri1 30, 1992, an Unreviewed Safety Question (USQ) concerning criticality safety issues in the Tank Farms was declared to be a reportable event. Unusual Occurrence report KL-W4C-TANKFARM-1992-0037 was issued. A prohibition was placed on all waste transfers into and between the tank farm facilities which is negatively affecting various Hanford programs. On September 1, 1992, the approved Justification For Continued Operations (JCO) was received. This establishes the limitations for all tanks receiving transfers and also excludes any interim stabilization of single-shell tanks until further evaluations are completed and approved by DOE-HQ. On December 15, 1992, the apprcived JCO was issued as WHC-SD-WM-JCO-001, "Justification for Continued Operations of Hanford High Level Waste Tanks Resulting From the Criticality USQ, 492-CRITSAS." An amendment request to this JCO to pump and stabilize Tank 241-T-101 has been sent to DOE-HQ for approval. The resolution of the USQ is scheduled for summer 1993.

\section{Saltwell Pumping}

Interim stabilization has been delayed because of the criticality safety issues which suspended waste transfers into and between tank farm facilities. The approved Justification For Continued Operations (JCO) excludes any stabilization of single-shell tanks until further evaluations are completed and approved by DOE-HQ. The resolution of the USQ is scheduled for summer 1993.

\section{Tank 241-SY-101 Gas Venting}

Several maintenance and mitigation projects are scheduled for "Window $\mathrm{H}$," following the next venting of Tank 101-SY, which is expected to occur in February. The key activity will be the installation of a mixer pump which may bring an end to the periodic venting. The goal is to create a continuous release of gases with the least amount of agitation of the waste. This will reduce the likelihood of an episodic venting in potentially explosive concentrations.

\section{Occurrence Reports}

RL-WHr. TANKFARM-1993-0005 (UNUSUAL) - MISSED SURVEILLANCE READINGS ON TANK 106-C VACUUM RESULTS IN A NON-CONFORMANCE TO OSR-T-152-00003 (Notification Report, January 12, 1993) On January 12, 1993, at 0430 hours, it was discovered that missed vacuum surveillance readings for the 105/106-C exhauster resulted in a Nonconformance to the OSR. The surveillance requirement is for vacuum readings to be obtained "daily, not to exceed 30 hours." Twelve hours elapsed beyond the 30-hour requirement before vacuum readings were obtained. Snow and icy conditions prevailed, with ambient temperatures below $15^{\circ} \mathrm{F}$. Access to farm required supplied breathing air for entry. 
Entry steps were cleared of snow and ice to 241-C farm at 0445 hours and readings were obtained at 0500 hours on January 12.

RL-WHC-TANKFARM-1993-0012 (UINUSUAL) - POTENTIIAL PERSONNEL EXPOSURE TO HAZARDOUS VAPORS (Notification Report, January 23, 1993)

On January 21, 1993, at 2030 hours, four workers in 241-sy farm were potentialiy exposed to hazardous vapors in excess of the occupational safety limit. The foc $r$ workers were in a greenhouse, attempting to install a drain line on one of the over pack assemblies for the Tank 101-sY lances. The workers were removing a one-inch pipe plug and noted a strong ammonia ordor. The workers immediately evacuated the greenhouse. At 2050 hours, one of the workers exited the farm complaining of burning eyes, sore throat and a headache. He was sent to first aid for examination and returned to the work site. This worker had been approximately four feet from the pipe opening. The other three workers in the greenhouse reported no adverse effects at 2055 hours. The area was placed in a safe configuration at 2100 hours.

On January 22, at 1530 hours, shift supervision was notified that industrial hygiene personnel checked the vapor conditions within the air lance receivers and found them to be at levels $>700 \mathrm{ppm}$. The levels the the personnel were exposed to will still need to be determined. The investigation is continuing.

RL-WHC-TFARM-1993-0014 (OFF-NORMAL) - TRANSFER OF COMPLEXED WASTE FROM 204-AR TO 101-AY RESULTED IN OUT-OF-TOLERANCE MATERIAL BALANCE AND UNEXPECTED INCREASE IN 102 -AY. (Not ification Report, January 26, 1993) On January 25, 1993, at 2120 hours, a transfer from 204-AR Rail Car Facility to Tank 101-AY was started. At 2132 hours, a leak detector in $A X-B$ Valve Pit activated, shutting down the transfer pump. At 2135 hours, the leak detector cleared. At 2145 hours, all catch tanks and interconnected tanks were checked and no level differences were noted. At 2155 hours, the transfer was restarted and no further alarms were received on any leak detectors for the duration of the transfer. The transfer and flush were completed at 2330 hours.

After a 2-hour drain-down period, final data was obtained. Initial calculation of the material balance showed a discrepancy of -0.65 inches in the receiving tank which exceeds the all lowable tolerance of \pm 0.5 inches. It was also noted that Tank 102-AY, which served as both an interconnecting tank and as a valve pit leakage catch tank for the transfer, showed an apparent level rise of 0.4 inches ( 1100 gallons). All data was verified to be accurate in the field and the flush volumes were carefully checked for proper accountability in the material balance. On January 26, at 0445 hours, the material balance was completed and the discrepancy was confirmed to be -0.65 inches.

At 0545 hours, a limited inspection of the AX-B Valve Pit was performed through an inspection port in the cover block. The inspection indicated that the floor of the pit was wet. The investigation is continuing. 
Changes to the Report:

1. Summary section

Tables 2 through 5 (Temperature Tables): The table formats and footnotes have been revised. All temperatures shown in these tables were taken in the waste unless otherwise indicated.

Figures 3 and 4 (Single-Shell Tanks Waste Volumes Grouped by Waste Types) show a graphic representation of the waste in the tanks for Sound and Assumed Leaking tanks. These figures are presented in this issue only. 
WHC-EP-0182-58
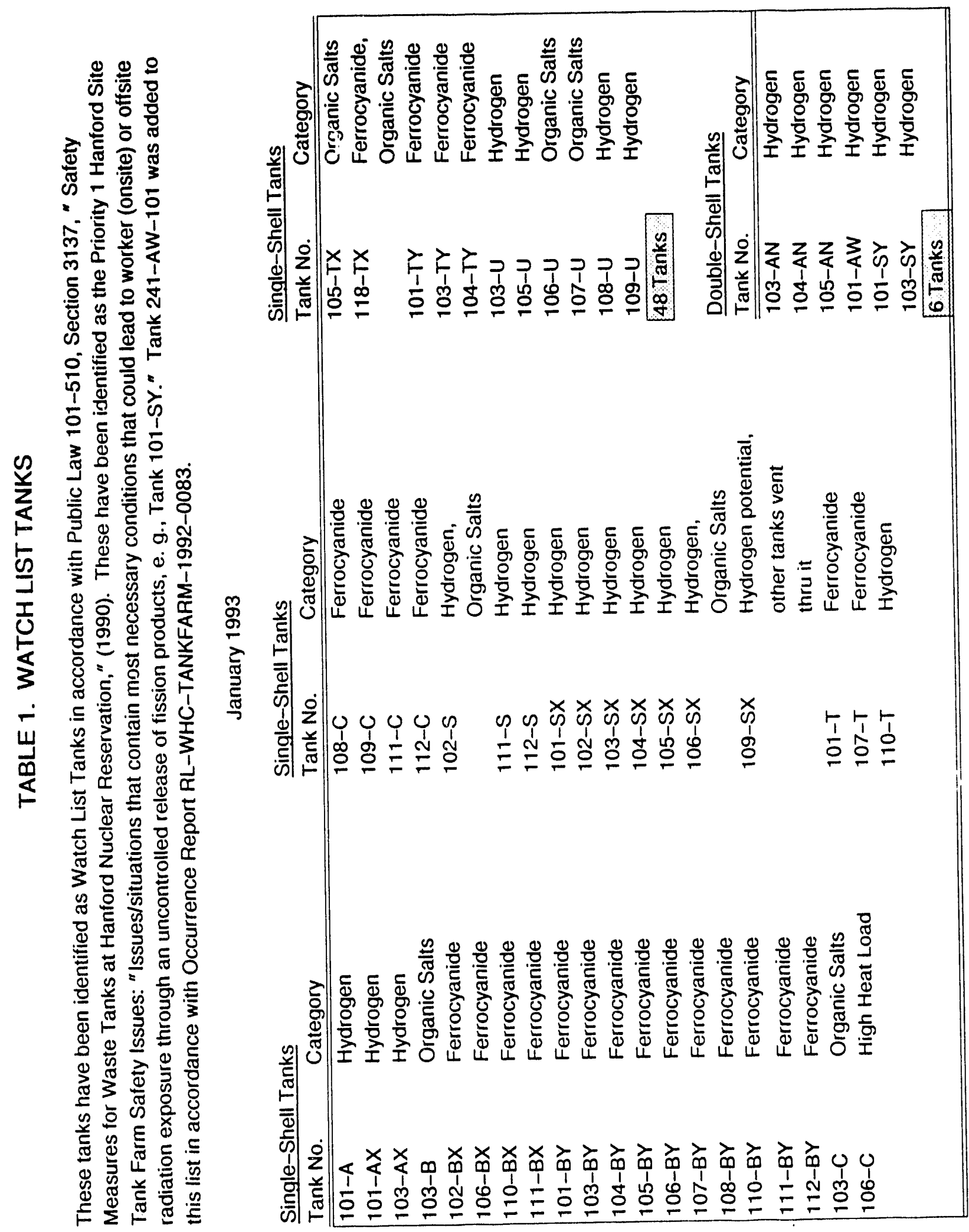
WHC-EP-0182-58

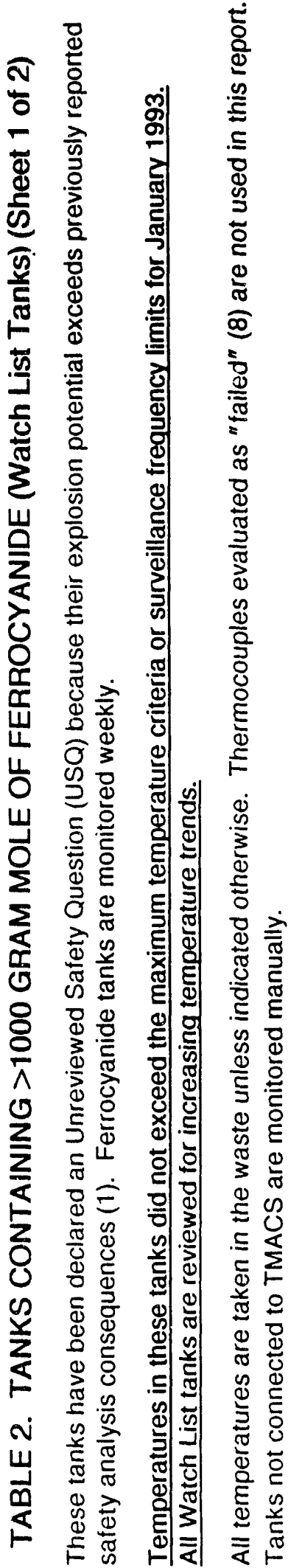

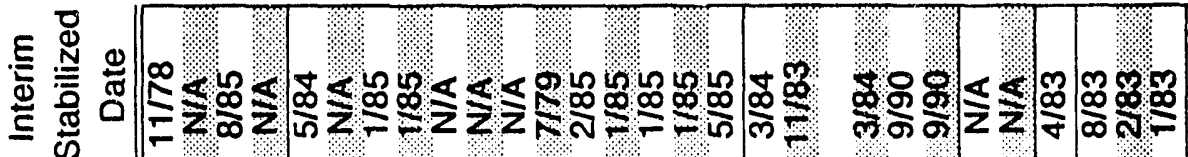

¿

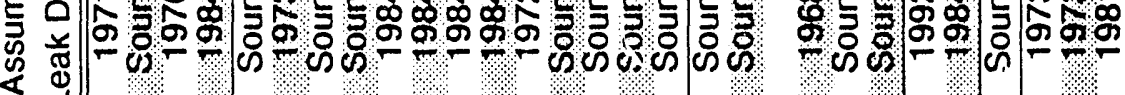

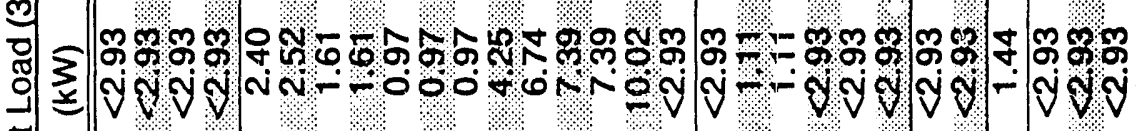

.

I

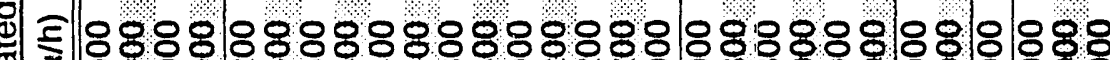

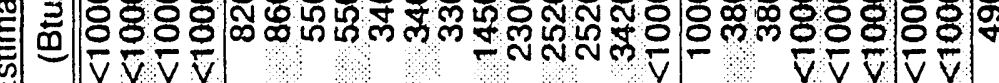

ш)

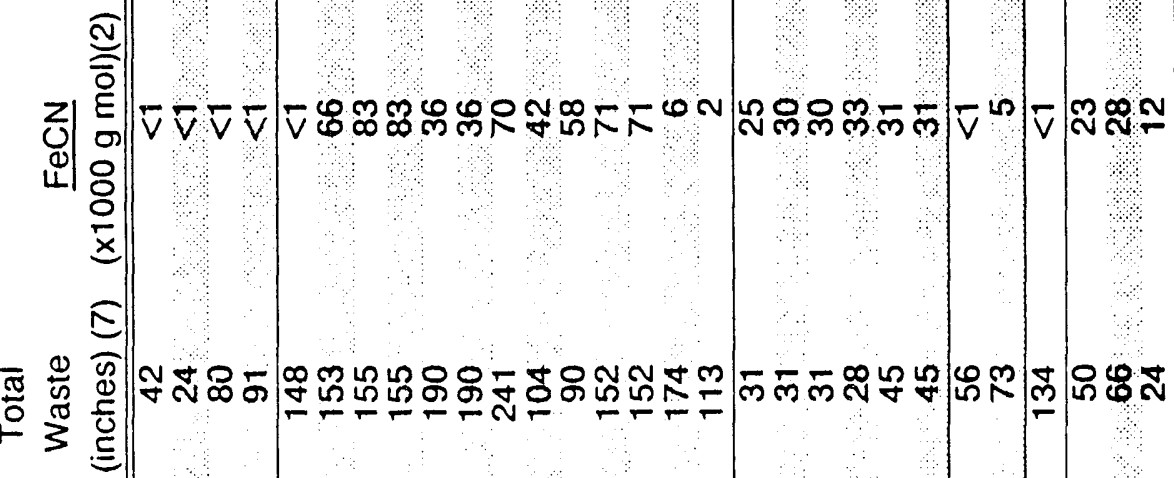

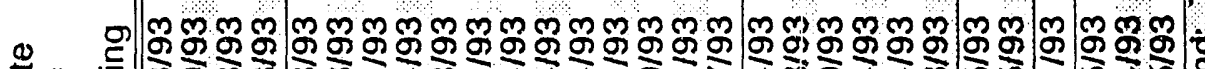
E.

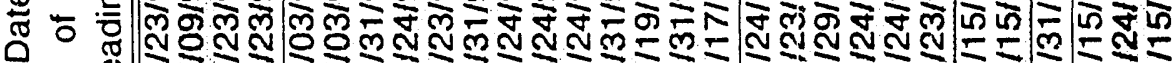
d

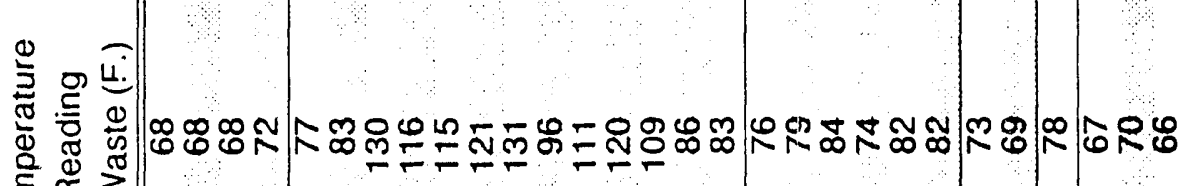

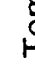




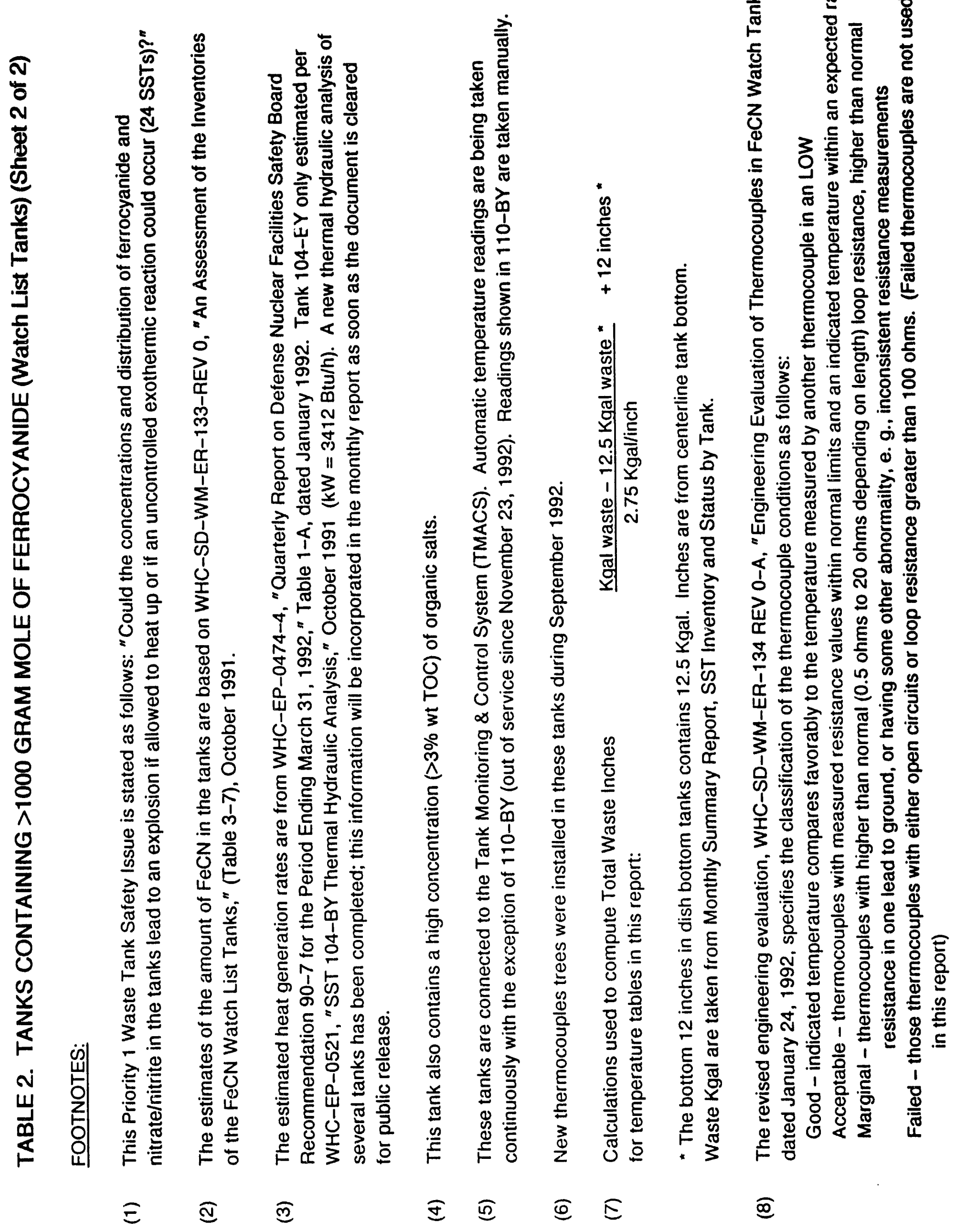




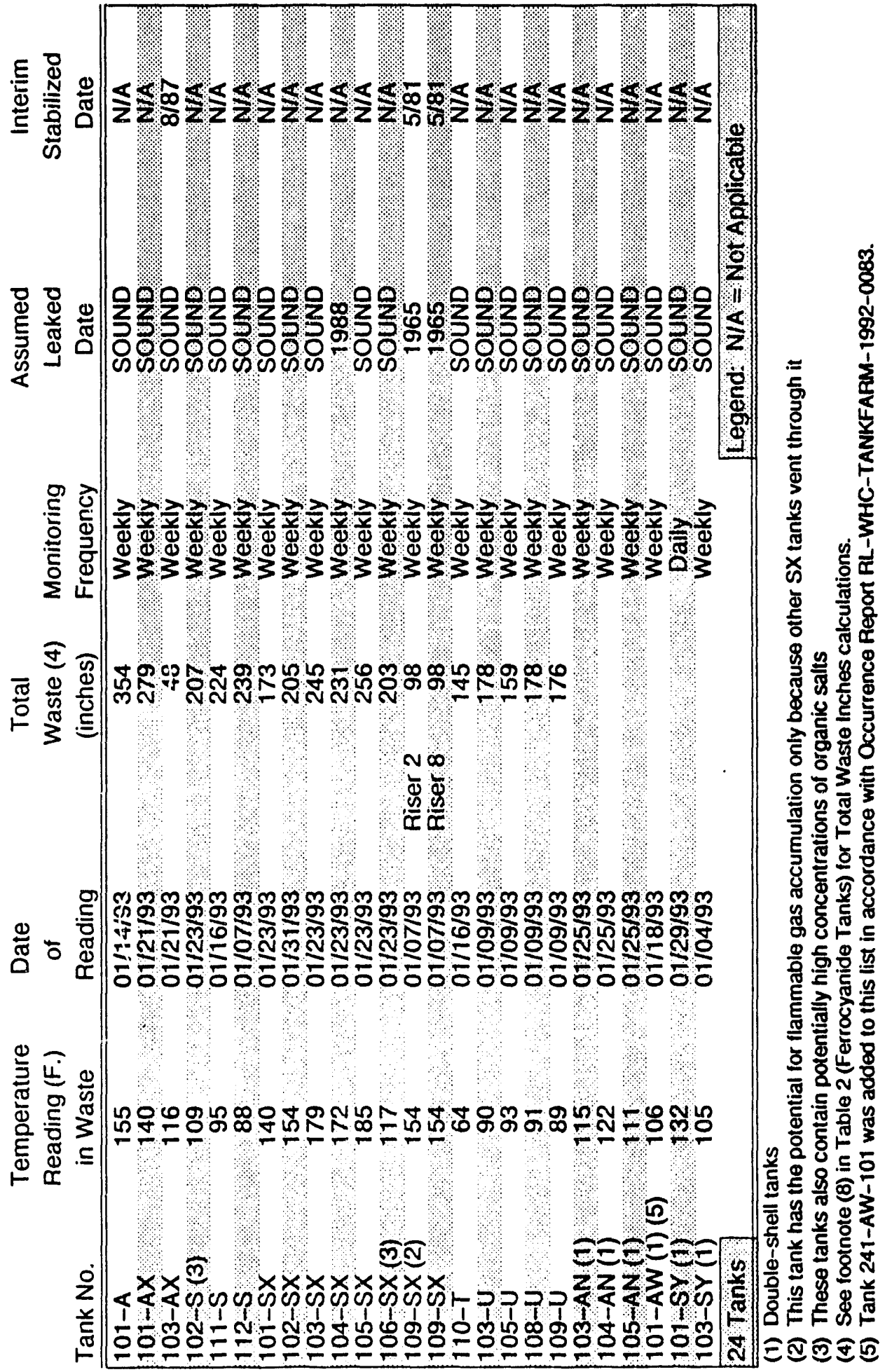


ङ

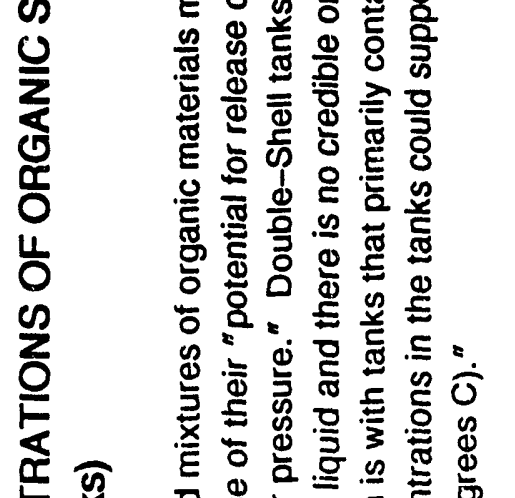

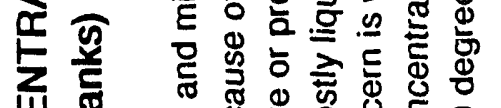

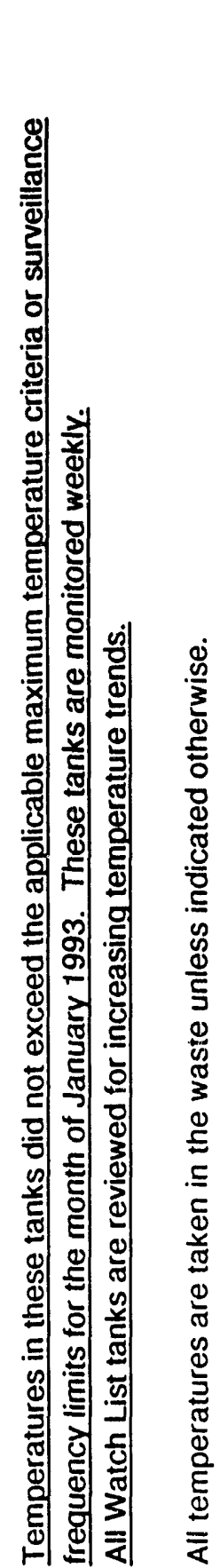

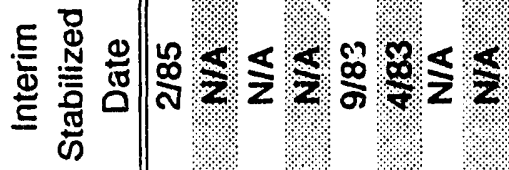

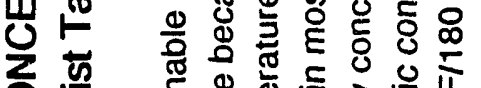

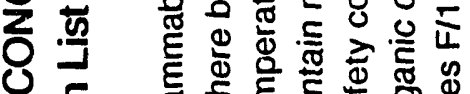

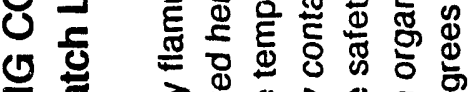

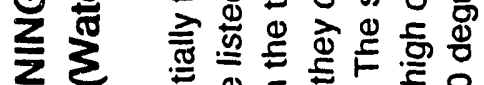

Z

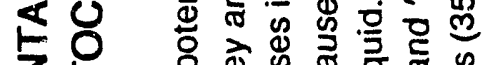

Z능 đ

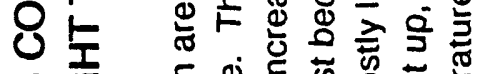

అ 志

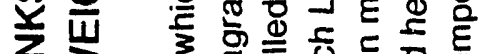

《了

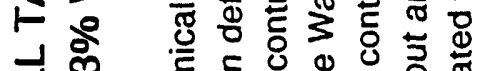

Ш

ज

山

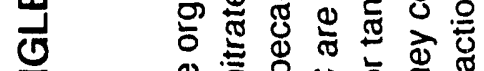

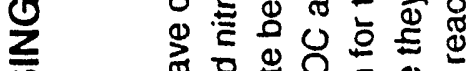

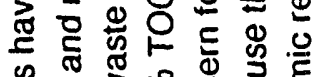

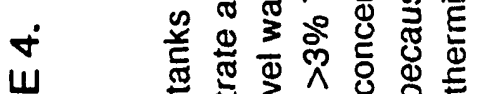

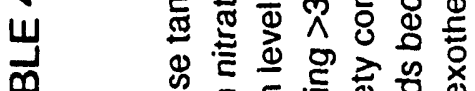

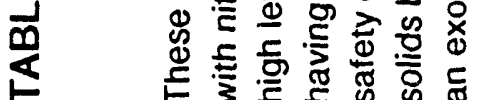

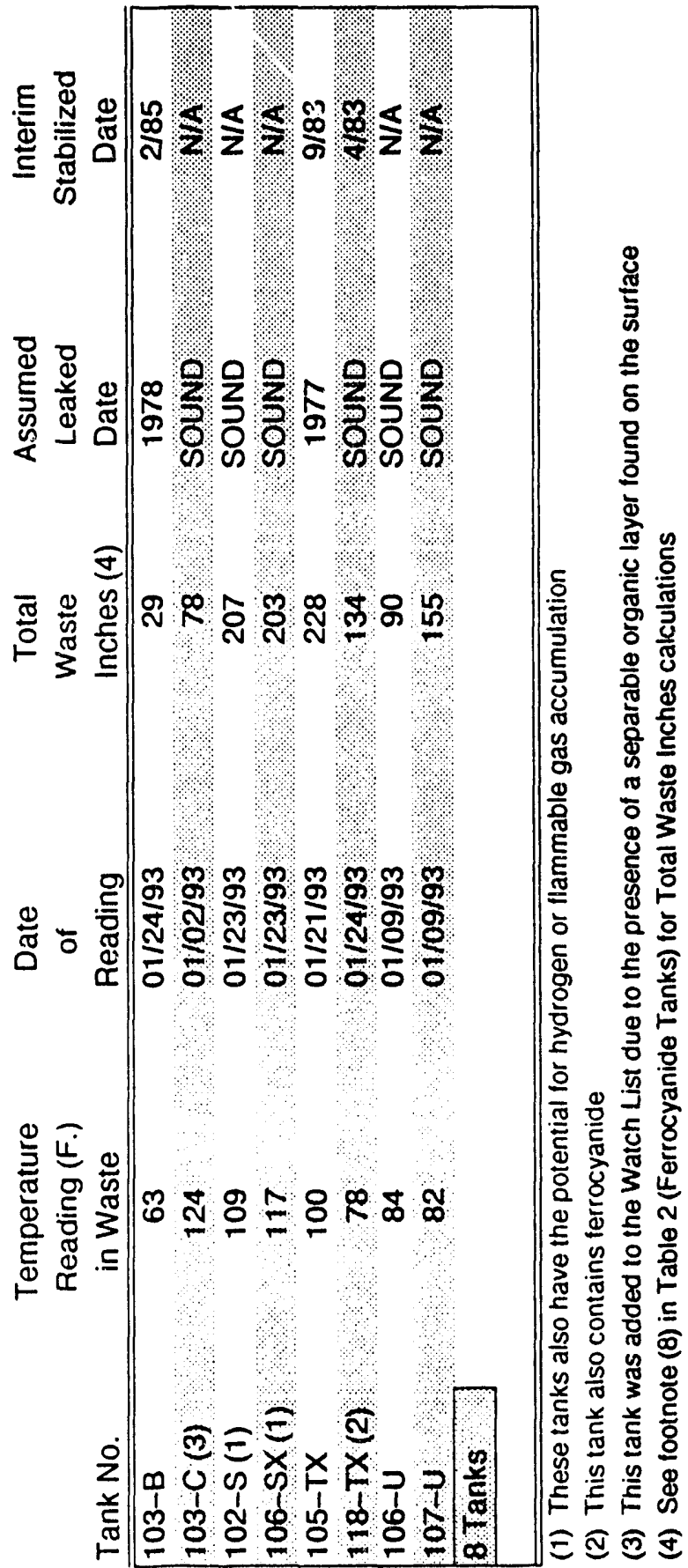




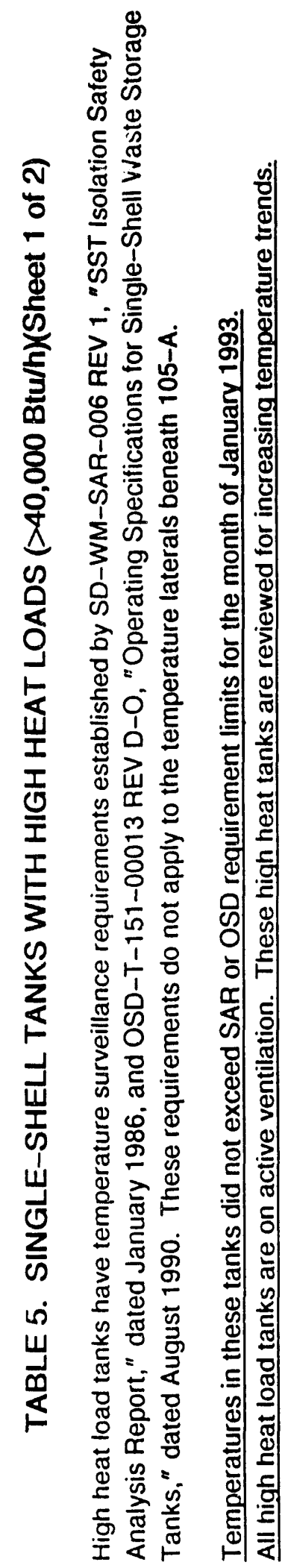

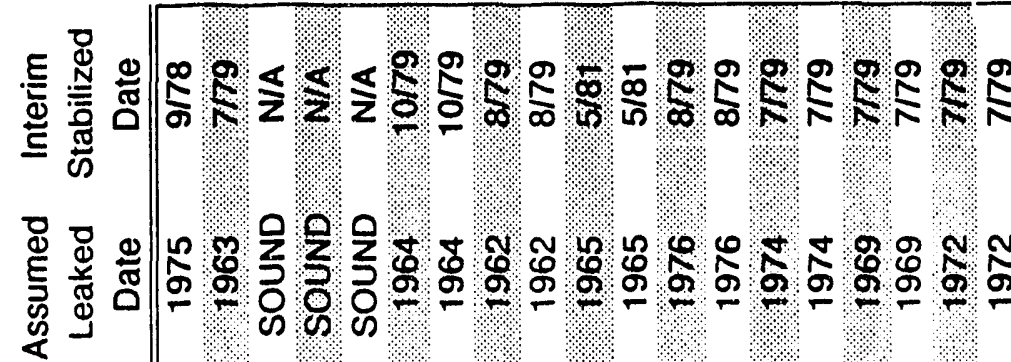

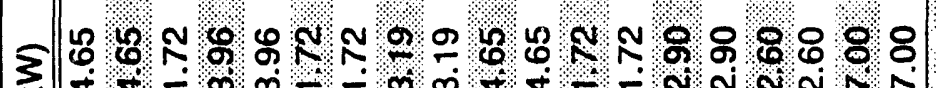

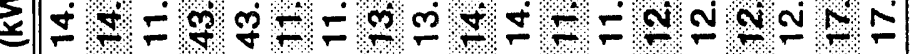

Ф़)

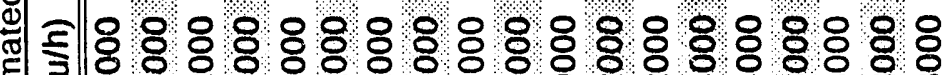

绨品

\section{g}

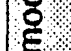

ह

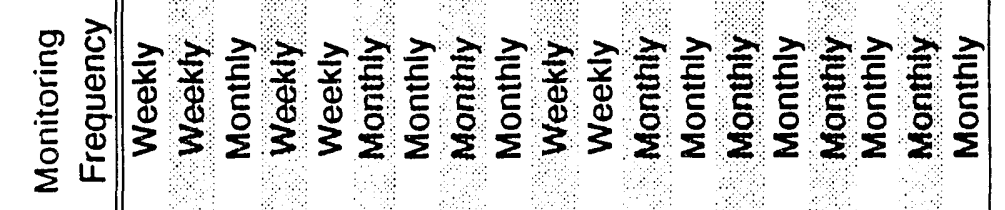

ำ

Eิ

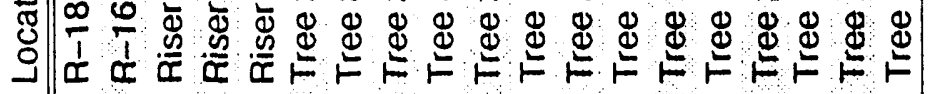

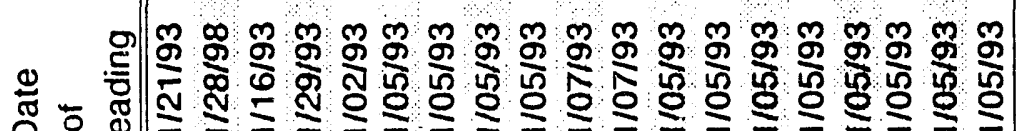

范

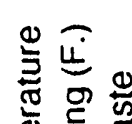

递要

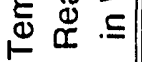

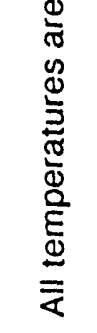
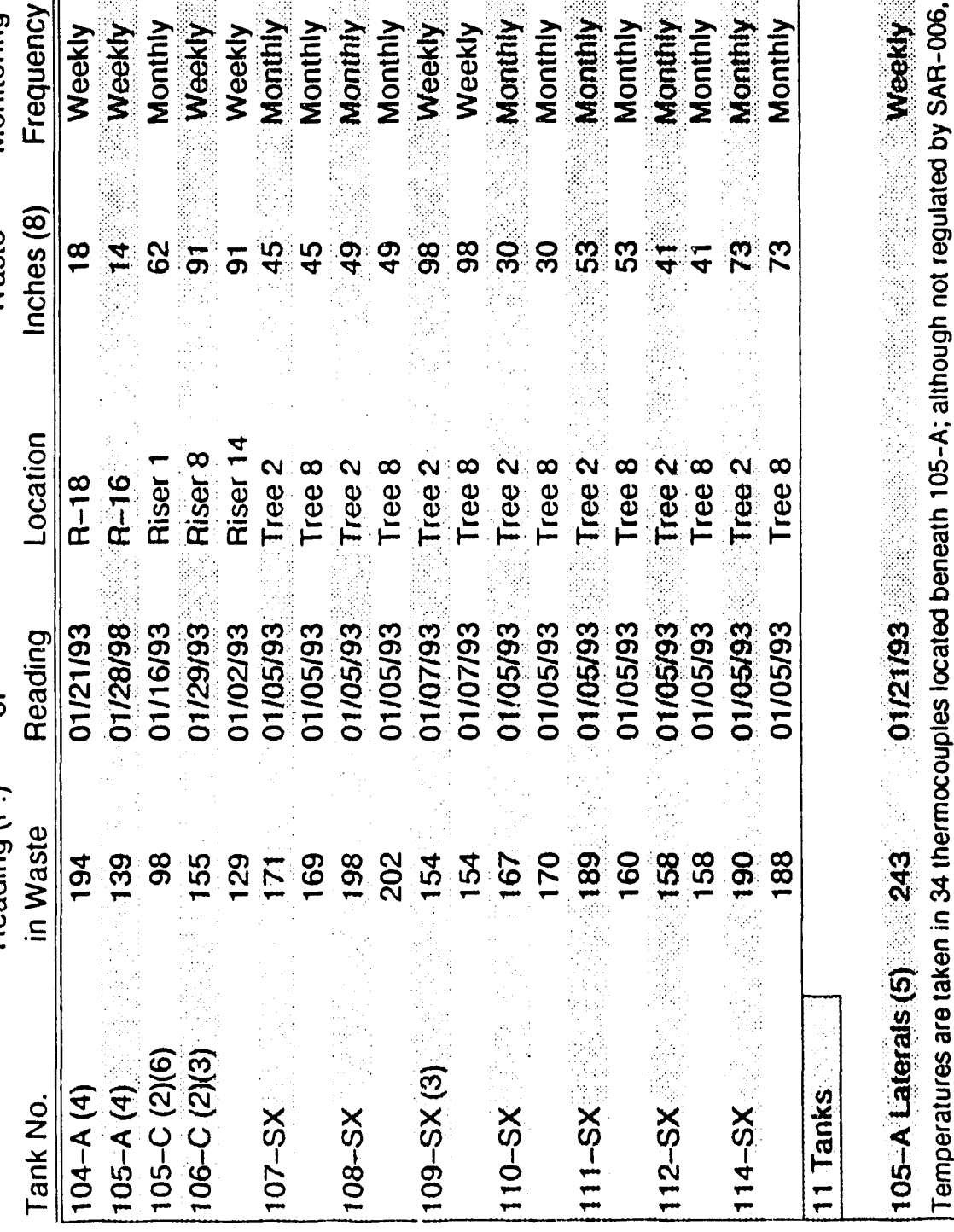


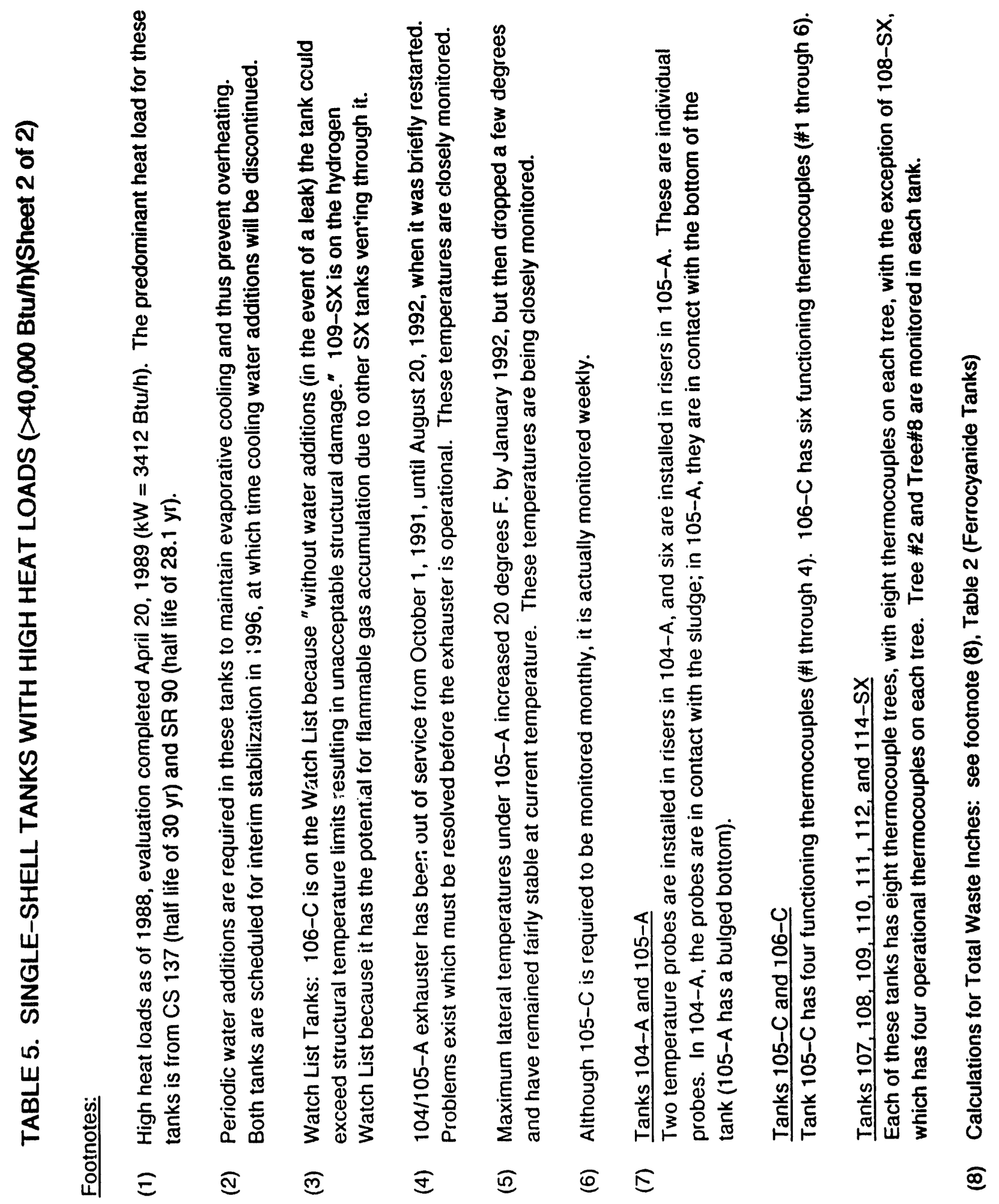


WHC-EP-0182-58

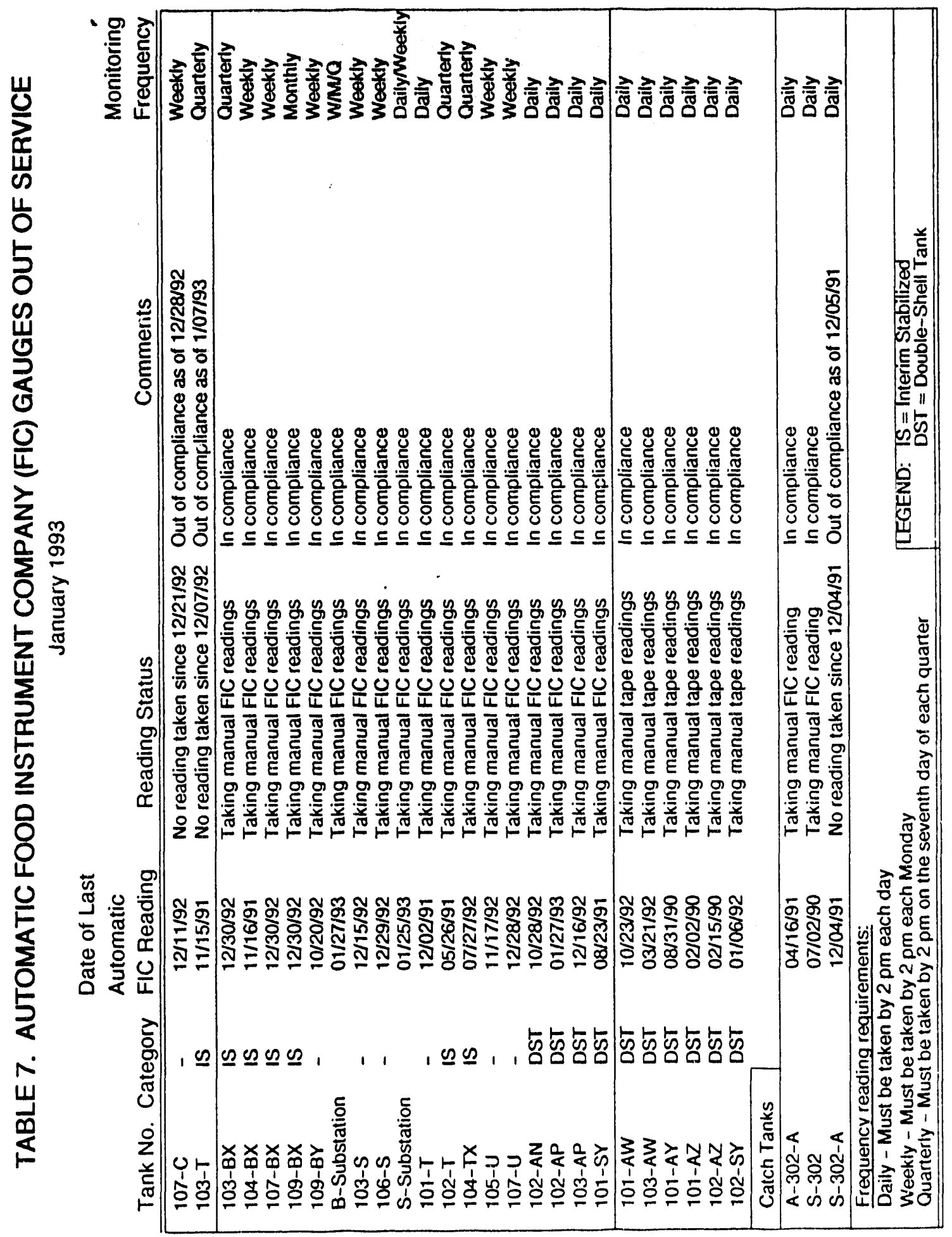




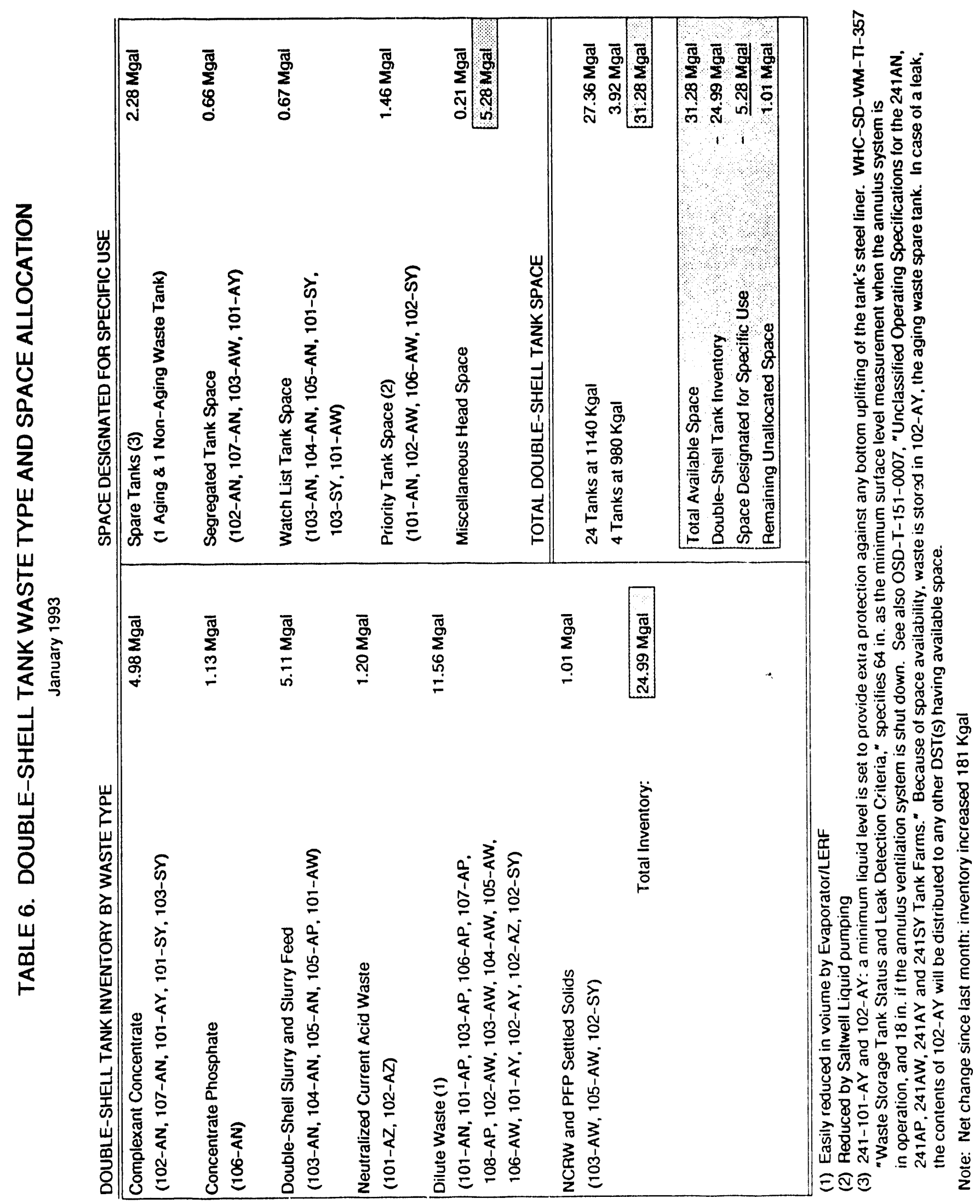




\section{TABLE 8: SINGLE-SHELL TANKS MONITORING COMPLIANCE STATUS 149 TANKS (Sheet 1 of 4 )}

The following tabie indicates whether Single-Shell tank monitoring is in compliance with the requirements as specified in the applicabie Jocuments:

All Watch List and High Heat tank temperature monitoring is in compliance. compiiance.

All Drywell monitoring is in compliance.

Psychrometrics (2)

In-tank Photographs (3)
NOTE:

All Dome Elevation Survey monitoring is in

\begin{tabular}{|c|c|}
\hline $\begin{array}{l}\text { LEGEND: } \\
\text { (Shadod) }\end{array}$ & = In compliance with all applicable documentation \\
\hline O/C & $=$ Out of compliance with documentation \\
\hline-357 & $\begin{aligned}= & \text { WHC-SD-WM-TI-357. "Waste Storage Tank Status and Leak } \\
& \text { Detection Criteria" }\end{aligned}$ \\
\hline POP & $\begin{array}{l}=\text { Plant Operating Procedure TO-040-650, "Obtain/Record SST } \\
\text { Temperatures" }\end{array}$ \\
\hline M.T. & $=$ Manual Tape \\
\hline FIC & z Food Instrument Company \\
\hline OSRUSAR & $\begin{aligned}= & \text { Operations Safety Requirements/Safety Analysis Report } \\
& \text { Requirements }\end{aligned}$ \\
\hline Oso & $=$ Operating Specifications Document \\
\hline N/A & $=$ Not Applicable (i.e., no LOW, M.T, FIC installed) \\
\hline O/S & $=$ Out of Service \\
\hline Gamma & $=L O W$ readings taken by Gamma probe \\
\hline Neutron & $=$ LOW readings taken by Neutron probe \\
\hline
\end{tabular}

Information as of $1 / 31 / 93$

\begin{tabular}{|c|c|c|c|c|c|c|c|c|c|c|}
\hline \multirow{4}{*}{$\begin{array}{l}\text { Tank } \\
\text { Number }\end{array}$} & \multirow{2}{*}{\multicolumn{2}{|c|}{ Category }} & \multirow{4}{*}{$\begin{array}{l}\text { Temperature } \\
\text { Readings (5) }\end{array}$} & \multirow{3}{*}{\multicolumn{2}{|c|}{$\begin{array}{l}\text { Surface Level } \\
\text { Readings (1) } \\
(-357)\end{array}$}} & \multirow{3}{*}{\multicolumn{2}{|c|}{$\begin{array}{l}\text { LOW Readings } \\
(-357)\end{array}$}} & \multicolumn{2}{|c|}{ Radiation Readings } & \multirow{4}{*}{$\begin{array}{c}\text { Dome } \\
\text { Elevation } \\
\text { Surveys } \\
\text { (OSR/SAR) }\end{array}$} \\
\hline & & & & & & & & \multirow{3}{*}{$\begin{array}{l}\text { Lateral } \\
\text { Readings } \\
(-357) \\
\end{array}$} & \multirow{3}{*}{\begin{tabular}{|c|} 
Drywell \\
Readings \\
(OSRSAR) \\
\end{tabular}} & \\
\hline & \multirow{2}{*}{$\begin{array}{c}\text { Watch } \\
\text { List }\end{array}$} & \multirow{2}{*}{$\begin{array}{l}\text { High } \\
\text { Heat }\end{array}$} & & & & & & & & \\
\hline & & & & M.T. & FIC & Gamma & Neutron & & & \\
\hline $101-A$ & $x$ & & 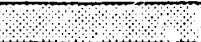 & & N/A & & 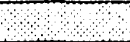 & O/C & & ४ \\
\hline $102-A$ & & & & NIA & $1 \%$ & NIA & NIA & $\mathrm{O} / \mathrm{C}$ & & \\
\hline 103-A & & & & NUA & & & & $\mathrm{O} / \mathrm{C}$ & & $\%$ \\
\hline 104-A & & $x$ & 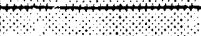 & $O / C$ & $N / A$ & N/A & N/A & O/C & 6 & एम \\
\hline 105-A & & $x$ & & ২ে & NIA & N/A & N/A & $\mathrm{O} / \mathrm{C}$ & & \\
\hline $106-A$ & & & & $N A A$ & & N/A & N/A & O/C & & \\
\hline $101-A \bar{X}$ & $\bar{x}$ & & & N/A & & & & & & म \\
\hline $102-A X$ & & & O/C & & N/A & N/A & NIA & & & \\
\hline $103-A \bar{X}$ & $\bar{x}$ & & & NIA & & N/A & NIA. & & & \\
\hline$\overline{104-A X}$ & & & & $\mathrm{O} / \mathrm{C}$ & N/A & N/A & N/A & & & + \\
\hline $101-B$ & & & & N/A & 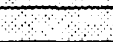 & N/A. & N/A & & & \\
\hline $102-B$ & & & 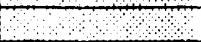 & NIA & 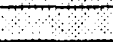 & N/A & N/A & & & + \\
\hline $103-B$ & $\bar{x}$ & & & NIA & & NIA & NIA & & & \\
\hline $104-B$ & & & & & N/A & 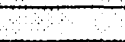 & + & & & बि \\
\hline $105-B$ & & & & & N/A & & & & & 3 \\
\hline $106-B$ & & & & NIA & & N/A & N/A & & & एक \\
\hline $107-8$ & & & & & $N / A$ & $N / A$ & N/A & & & \\
\hline $108-8$ & & & & N/A & & N/A & N/A & $\therefore$ & & \\
\hline $109-\bar{B}$ & & & & & N/A & N/A & N/A & & & \\
\hline $110-8$ & & & O/C & $\mathrm{O} / \mathrm{C}$ & N/A & N/A & N/A & & & 8 \\
\hline $111-8$ & & & O/C & N/R & & N/A & N/A & & & \\
\hline $112-B$ & & & 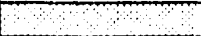 & N/A & & N/A & N/A & & & \\
\hline $201 \ldots-B$ & & & & 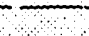 & N/A & NIA & N/A & & & 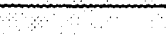 \\
\hline 202-B & & & -7 & 7 & N/A & N/A & N/A & & & \\
\hline $203-B$ & & & & & N/A & N/A & N/A & & & \\
\hline $204-B$ & & & & & N/A & N/A & N/A & & & \\
\hline $101-B X$ & & & O/C & 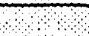 & NIA & N/A & N/A & & & \\
\hline $102-8 x$ & $x$ & & & & N/A & N/A & N/A & & & \\
\hline $103-B X$ & & & O/C & NIA & & N/A & NIA & & & \\
\hline $104-B X$ & & & O/S-O/C & N/A & & N/A & N/A & & & 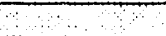 \\
\hline $105-8 X$ & & & & NIA & & N/A & N/A & & & \\
\hline $106-8 X$ & $\bar{x}$ & & & $N / A$ & & N/A & N/A & & & \\
\hline $107-B X$ & & & $\mathrm{OIC}$ & N/A & & NIA & NIA & & & \\
\hline
\end{tabular}


TABLE 8. SINGLE-SHELL TANKS MONITORING COMPLIANCE STATUS 149 TANKS (Sheet 2 of 4 )

Information as of $1 / 31 / 93$

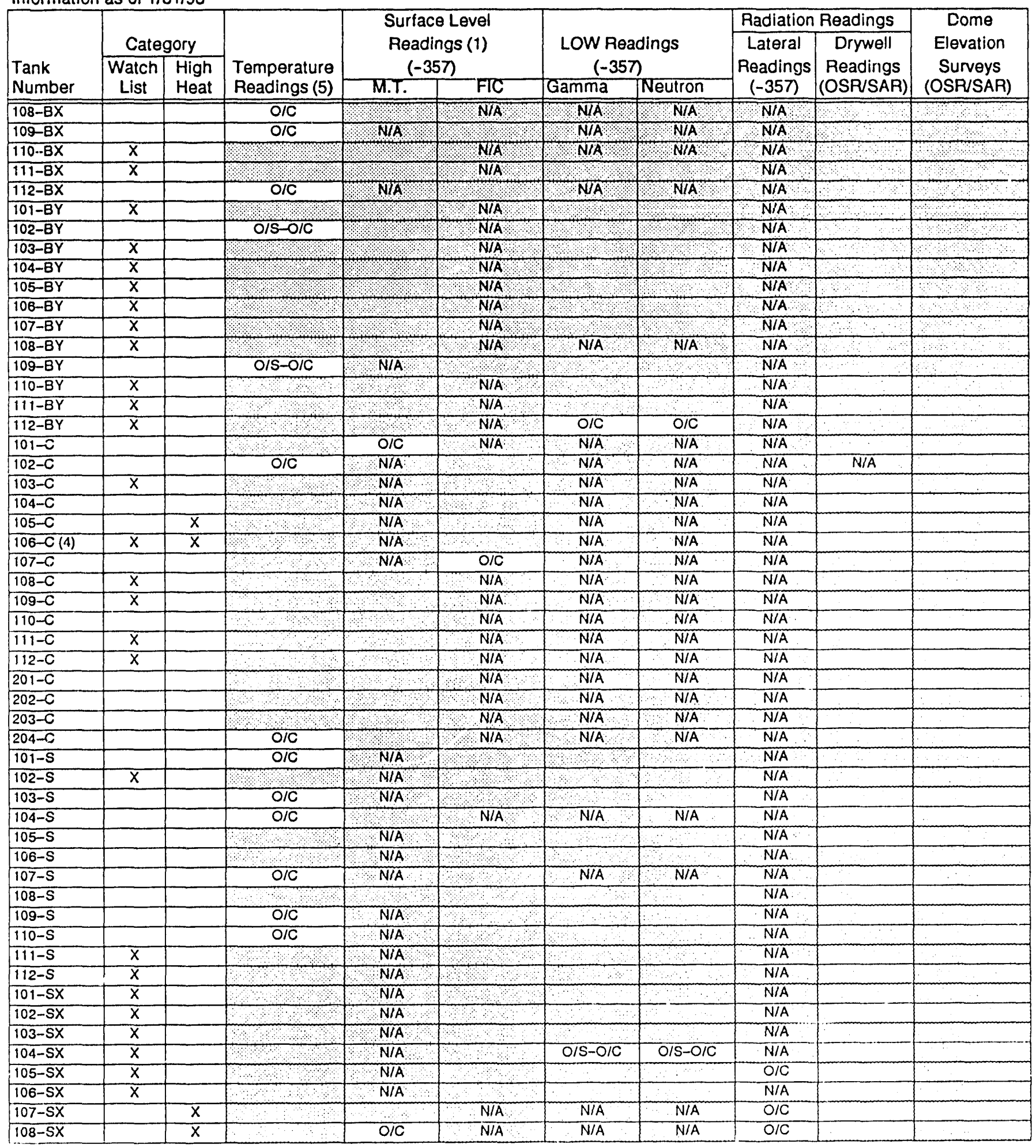


TABLE 8. SINGLE-SHELL TANKS MONITORING COMPLIANCE STATUS 149 TANKS (Sheet 3 of 4 )

Information as of $1 / 31 / 93$

\begin{tabular}{|c|c|c|c|c|c|c|c|c|c|c|}
\hline \multirow{4}{*}{$\begin{array}{l}\text { Tank } \\
\text { Number }\end{array}$} & \multirow{2}{*}{\multicolumn{2}{|c|}{ Category }} & \multirow{4}{*}{$\begin{array}{l}\text { Temperature } \\
\text { Readings (5) }\end{array}$} & \multirow{3}{*}{\multicolumn{2}{|c|}{$\begin{array}{l}\text { Surface Level } \\
\text { Readings (1) } \\
(-357)\end{array}$}} & \multirow{3}{*}{\multicolumn{2}{|c|}{$\begin{array}{l}\text { LOW Readings } \\
\qquad(-357)\end{array}$}} & \multicolumn{2}{|c|}{ Radiation Readings } & \multirow{4}{*}{$\begin{array}{c}\text { Dome } \\
\text { Elovation } \\
\text { Surveys } \\
\text { (OSR/SAR) }\end{array}$} \\
\hline & & & & & & & & \multirow{3}{*}{$\begin{array}{c}\text { Lateral } \\
\text { Readings } \\
(-357) \\
\end{array}$} & \multirow{3}{*}{\begin{tabular}{|c|} 
Drywell \\
Readings \\
(OSR/SAR)
\end{tabular}} & \\
\hline & \multirow{2}{*}{$\begin{array}{c}\text { Watch } \\
\text { List }\end{array}$} & \multirow{2}{*}{$\begin{array}{l}\text { High } \\
\text { Heat }\end{array}$} & & & & & & & & \\
\hline & & & & М.T. & FIC & Gamma & Neutron & & & \\
\hline $109-5 \times(4)$ & $\mathrm{x}$ & $x$ & & & NIA & NIA & NIA & $\mathrm{OIC}$ & & . \\
\hline $110-5 X$ & & $\bar{x}$ & & & NIA & $N / A$ & $N / A$ & $\mathrm{O} / \mathrm{C}$ & & \\
\hline $111-\mathrm{SX}$ & & $\bar{x}$ & & & $\mathrm{NA}$ & $\mathrm{N} / \mathrm{H}$ & $\mathrm{N} / \mathrm{A}$ & $\mathrm{O} / \mathrm{C}$ & & \\
\hline $112-5 X$ & & $\bar{x}$ & & & NIA & NA & NIA & $\mathrm{OIC}$ & & \\
\hline $113-5 X$ & & & $O / C$ & & NA & NA & $N 1 \mathrm{~A}$ & NIA & & \\
\hline $114-5 x$ & & $x$ & & & NIA & NIA & NIA & O/C & & \\
\hline $115-s x$ & & & $\mathrm{O} / \mathrm{S}-\mathrm{OIC}$ & & NIA & N/A & N/A & $\mathrm{O} / \mathrm{C}$ & & \\
\hline $101-T$ & $x$ & & & NIA & & NIA & $N / A$ & NIA & & \\
\hline $102-T$ & & & O/S - O/C & NIA & & $N / A$ & $N / A$ & N/A & & \\
\hline 103-T & & & $\mathrm{O} / \mathrm{C}$ & $N / A$ & $\mathrm{O} / \mathrm{C}$ & NIA & NIA & NIA & & \\
\hline $104-T$ & & & O/C & & NIA & & $\mathrm{O} / \mathrm{C}$ & N/A & & $\therefore$ \\
\hline $105-T$ & & & $\mathrm{O} / \mathrm{S}-\mathrm{O} / \mathrm{C}$ & NIA & & NIA & NIA & N/A & & \\
\hline $106-T$ & & & O/C & NIA & & N/A & NIA & $\mathrm{NIA}$ & & \\
\hline $107-T$ & $x$ & & & N/A & & NIA: & $\mathrm{N} / \mathrm{A}$ & NIA & & \\
\hline $108-T$ & & & O/C & & NIA & NIA & N/A & N/A & & \\
\hline $109-\mathrm{T}$ & & & $\mathrm{O} / \mathrm{C}$ & NIA & का & NIA & NIA & N/A & & \\
\hline $110-T$ & $x$ & & 0 & N/A & $\mathrm{L}$ & $\therefore$ & $\mathrm{O} / \mathrm{C}$ & N/A & & 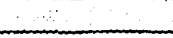 \\
\hline $111-T$ & & & $O / C$ & N/A & 4 & & $\mathrm{O} / \mathrm{C}$ & NIA & & \\
\hline $112-T$ & & & $\mathrm{O} / \mathrm{C}$ & N/A & $6 \ldots$ & NIA & NIA & NIA & & \\
\hline $201-T$ & & & O/C & & N/A & NIA & NIA & $\mathrm{N} / \mathrm{A}$ & & \\
\hline $202-T$ & & & $\mathrm{O}$ & 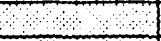 & $N / A$ & NIA & $N / A$ & N/A & & \\
\hline $203-T$ & & & $\mathrm{O} / \mathrm{C}$ & & NIA & N/A & N/A & NIA & & \\
\hline $204-T$ & & & $\mathrm{O} / \mathrm{C}$ & & NIA & NIA & $N / A$ & N/A & & \\
\hline $101-\mathrm{TX}$ & & & $O / S-O / C$ & N/A & & $N / A$ & NIA & NIA & & \\
\hline $102-T X$ & & & OIC & 8 & N/A & & $\mathrm{OIC}$ & N/A & & \\
\hline 103-TX & & & $\mathrm{O} / \mathrm{C}$ & NIA & & $\mathrm{N} / \mathrm{A}$ & N/A & NIA & & \\
\hline 104-TX & & & $\mathrm{O} / \mathrm{C}$ & N/A & & N/A & NIA & N/A & & \\
\hline 105-TX & $\bar{x}$ & & & म. & N/A & $\mathrm{O} / \mathrm{S}-\mathrm{O} / \mathrm{C}$ & OIS-OIC & N/A & & \\
\hline 108-TX & & & $\mathrm{OIC}$ & & N/A & & O/C & N/A & & \\
\hline $107-T X$ & & & $\mathrm{O} / \mathrm{C}$ & NIA & 3 & NIA & N/A & $N / A$ & & \\
\hline $108-\mathrm{TX}$ & & & $\mathrm{OIC}$ & NIA & + & 3 & $\mathrm{O} / \mathrm{C}$ & N/A & & \\
\hline 109-TX & & & $\mathrm{O} / \mathrm{C}$ & NIA & & 3 & O/C & N/A & 8 & \\
\hline $110-\mathrm{TX}$ & & & $\mathrm{O} / \mathrm{C}$ & & NIA & म. & $\mathrm{O} / \mathrm{C}$ & NIA & & \\
\hline $111-\mathrm{TX}$ & & & $\mathrm{O} / \mathrm{C}$ & & NIA & . & $\mathrm{O} / \mathrm{C}$ & NIA & & \\
\hline $112-T X$ & & & $\mathrm{O} / \mathrm{C}$ & & NIA & 3 & $\mathrm{O} / \mathrm{C}$ & N/A & & $\vdots$ \\
\hline $113-T X$ & & & $\mathrm{O} / \mathrm{C}$ & $\mathrm{O}$ & N/A & ? & $\mathrm{O} / \mathrm{C}$ & $N / A$ & & \\
\hline $114-\mathrm{TX}$ & & & $\mathrm{O} / \mathrm{C}$ & $\mathrm{n}$ & NIA & 3 & $\mathrm{O} / \mathrm{C}$ & N/A & & \\
\hline $115-T X$ & & & $\mathrm{O} / \mathrm{C}$ & & NIA & 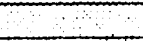 & $\mathrm{O} / \mathrm{C}$ & NIA & & \\
\hline $116-T X$ & & & O/SOIC & 4 & N/A & N/A & NIA & $N / A$ & & $\therefore$ \\
\hline $117-T X$ & & & $\mathrm{O} / \mathrm{C}$ & + & N/A & & $\mathrm{O} / \mathrm{C}$ & $N / A$ & & \\
\hline $118-\mathrm{TX}$ & $x$ & & प & NIA & $\therefore \quad$ & & $\mathrm{O} / \mathrm{C}$ & N/A & & \\
\hline $101-\mathrm{TY}$ & $x$ & & & N/A: & & NIA & N/A & NIA & & \\
\hline 102-TY & & & O/C & N/A & & N/A & N/A & $N / A$ & & . \\
\hline $103-\mathrm{TY}$ & $x$ & & 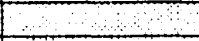 & NIA & $\ldots$ & & & NIA & & \\
\hline 104-TY & $x$ & & $\square \quad$ & N/A & +6 & $N / A$ & N/A & N/A & & \\
\hline 105-TY & & & $\mathrm{O} / \mathrm{C}$ & 7 & NIA & N/A & N/A & NIA & & \\
\hline 106-TY & & & $\mathrm{O} / \mathrm{C}$ & . & N/A & N/A & NIA & N/A & & \\
\hline $101-U$ & & & $\mathrm{O} / \mathrm{C}$ & $\square$ & N/A & NIA & NIA & $N / A$ & & \\
\hline $102-U$ & & & . & $\mathrm{N} / \mathrm{A}$ & , & 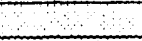 & & NIA & & \\
\hline $103-U$ & $x$ & & & $N / A$ & & + & & NIA & & $\therefore$ \\
\hline $104-U$ & & & O/S-O/C & . & $\mathrm{N} / \mathrm{A}$ & N/A & N/A & N/A & & \\
\hline $105-0$ & $x$ & & & NIA & & & & $\mathrm{N} / \mathrm{A}$ & & \\
\hline $106-U$ & $x$ & & $\ldots$ & $\mathrm{N} / \mathrm{A}$ & & & 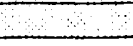 & N/A & & \\
\hline
\end{tabular}




\section{TABLE 8. SINGLE-SHELL TANKS MONITORING COMPLIANCE STATUS 149 TANKS (Sheet 4 of 4 )}

\begin{tabular}{|c|c|c|c|c|c|c|c|c|c|c|}
\hline \multirow{4}{*}{$\begin{array}{l}\text { Tank } \\
\text { Number }\end{array}$} & \multirow{2}{*}{\multicolumn{2}{|c|}{ Category }} & \multirow{4}{*}{$\begin{array}{l}\text { Temperature } \\
\text { Readings (5) }\end{array}$} & \multirow{3}{*}{\multicolumn{2}{|c|}{$\begin{array}{c}\text { Surface Level } \\
\text { Readings (1) } \\
(-357)\end{array}$}} & \multirow{3}{*}{\multicolumn{2}{|c|}{$\begin{array}{l}\text { LOW Readings } \\
(-357)\end{array}$}} & \multicolumn{2}{|c|}{ Radiation Readings } & \multirow{4}{*}{$\begin{array}{c}\text { Dome } \\
\text { Elevation } \\
\text { Surveys } \\
\text { (OSPUSAR) }\end{array}$} \\
\hline & & & & & & & & \multirow{3}{*}{$\begin{array}{c}\text { Lateral } \\
\text { Readings } \\
(-357)\end{array}$} & \multirow{3}{*}{\begin{tabular}{|c|} 
Drywell \\
Readings \\
(OSR/SAR)
\end{tabular}} & \\
\hline & Watch & High & & & & & & & & \\
\hline & List & Heat & & TM.T. & FIC & Gamma & Neutron & & & \\
\hline $107-U$ & $\bar{x}$ & & & $\sqrt{1 / 4}$ & & & & WNA & & \\
\hline $108-U$ & $\bar{x}$ & & & Nox & & & & N/A & & \\
\hline $102-U$ & $\bar{x}$ & & & SNA & & & & NIA & & \\
\hline $110-U$ & & & & NTA & & N/A & N/A & NA & & \\
\hline $111-U$ & & & & N/A & & & & $\mathrm{N} / \mathrm{A}$ & & \\
\hline $112-U$ & & & O/C & & NIA & NIA & NIA & N/A & & \\
\hline $201-U$ & & & O/C & & N/A & NIA: & NIA & NIA & & \\
\hline $202-U$ & & & $\overline{O / C}$ & & NIA & $N A$ & N/A & N/A & & \\
\hline $203-U$ & & & O/C & & NIA & NIA & N/A & N/A & & \\
\hline 204-U & & & O/C & & $\mathrm{N} / \mathrm{A}$ & NIA & N/A & N/A & & \\
\hline \multicolumn{11}{|c|}{ Catch Tanks } \\
\hline$A-302-A$ & N/A & N/A & NIA: & N/A & & NIA: & UसA. & N/A & N/A: & N/A \\
\hline$A-302-B$ & N/A & N/A & N/A & & NIA & NIA & IIA & NIA & N/A & $N / A$ \\
\hline 311-ER & N/A & N/A & NIA & N/A & & NIR & NIA & NIA & N/A & N/A \\
\hline Vent Sta. & N/A & N/A & N/A & & N/A & NIA & NIA & N/A & N/A & NIA: \\
\hline $151-A Z$ & N/A & N/A & NIA & N/A & & N/A & N/A & N/A & N/A & NIA \\
\hline $154-A Z$ & N/A & N/A & NIA & & $N / A$ & N/A & NIA & N/A & N/A & N/A : \\
\hline$s-302$ & N/A & N/A & N/A & N/A & & NIA & N/A & त्राA & N/A & NIA \\
\hline$S-302-A$ & N/A & N/A & N/A & N/A & $\mathrm{O} / \mathrm{C}$ & NIA & N/A & N/A & N/A & N/A \\
\hline$T X-302-B$ & N/A & N/A & NIA & O/C & N/A & NIA & NIA & NIA & N/A & N/A \\
\hline$T X-302-C$ & N/A & N/A & N/A & N/A & & N/A & N/A & NIA & N/A & N/A \\
\hline $\mathrm{U}-301-\mathrm{B}$ & N/A & N/A & N/A & N/A & & N/A & NIA & N/A & N/A & N/A \\
\hline$U X-302-A$ & N/A & N/A & N/A & NIA & & N/A & NIA & N/A & N/A & N/A \\
\hline $141-S$ & N/A & N/A & N/A & & NIA & N/A & N/A & N/A & N/A & N/A \\
\hline $142-S$ & N/A & N/A & N/A & & N/A & N/A & NIA & N/A & N/A & NIA \\
\hline \multirow{3}{*}{$\begin{array}{l}\text { Totals: } \\
149 \text { tanks }\end{array}$} & \multirow{3}{*}{$\begin{array}{l}48 \\
\text { Watch } \\
\text { List } \\
\text { Tanks } \\
\text { (4) }\end{array}$} & \multirow{3}{*}{$\begin{array}{l}11 \\
\text { High } \\
\text { Heat } \\
\text { Tanks } \\
\text { (4) }\end{array}$} & \multirow{3}{*}{$\begin{array}{l}\text { O/C: } \\
61 \text { tanks - } \\
\text { (semiannual } \\
\text { monitoring } \\
\text { frequency) (5) }\end{array}$} & \multirow{3}{*}{$\begin{array}{l}\text { O/C: } \\
6 \text { tanks }\end{array}$} & \multirow{3}{*}{$\begin{array}{l}\text { O/C: } \\
3 \text { tanks }\end{array}$} & O/C: & O/C: & \multirow{3}{*}{$\begin{array}{l}O / C: \\
15 \text { tanks }\end{array}$} & \multirow[t]{3}{*}{0} & \multirow[t]{3}{*}{0} \\
\hline & & & & & & & & & & \\
\hline & & & & & & \multicolumn{2}{|c|}{$\begin{array}{c}58 \text { tanks have LOWs } \\
\text { (2 are } O / S)\end{array}$} & & & \\
\hline
\end{tabular}

(1) All SSTs have either manual tape or FIC, with the exception of 108-S which has both. All SST FICs are connected to CASS, (with the exception of 106-BX); however, the connection for many tanks is broken. For such cases, manual field readings are taken. Manual surface level readings include readings taken by manual tape, manual FIC (not connected to Computer Automated Surveillance System, 106-BX), manual readings of automatic FIC (if CASS is printing " 0 "), or automatic FIC. In some cases, the surface level readings are taken using a zip cord. While less accurate, such readings are acceptable for meeting the surface level reading requirements.

(2) Psychrometric readings are taken on tanks with active exhausters (High heat tanks 104/105-A, 105/106-C, 107, 108, 109, $110,111,112$, and 114-SX). Psychrometrics were not taken from 9/91 to 9/92. Exhauster down on 104/105-A since 10/1/91. 105/106-C have resumed psychrometric readings on a monthly frequency. Psychrometric frequency not specified in -357 or operating procedures.

(3) In-tank Photographs are not required by -357. Last in-tank photographs in SSTs were taken September 1990.

(4) Two tanks are on both category lists (106-C, 109-SX).

(5) Temperature readings may be regulated by OSD, -357 , or POP. Additionally, high heat load tanks are regulated by OSPUSAR. Thermcouples in the 9 tanks designated O/S-O/C are out of service; there are either no thermocouple probes in these tanks, or probes have been cut off, covered over, or are otherwise not functioning. The OSD does not require readings or repair of out-of-service thermocouples for the 92 non-heat load $(>40,000 \mathrm{Btu} / \mathrm{hr})$ tanks. However, the operating procedure (POP) requires that attempts are to be made semiannually in January and July to obtain readings from these tanks. 61 tanks are O/C because either no attempts were made to read them in January 1993 or readings could not be obtained. (102-AX had only one thermocouple working in January and July 1ऽ?? - it was not working in January 1993). 
WHC-EP-0182-58

\section{TABLE 9. DOUBLE-SHELL TANKS MONITORING COMPLIANCE STATUS} 28 TANKS

The following table indicates whether Double-Shell tank monitoring is in compliance with the requirements as specified in the applicable documents:

\section{NOTE:}

All DST tank temperature tree monitoring is in compliance.

Dome Elevation Surveys are not required for DSTs.

Psychrometrics (2)

In-tank Photographs (3)

\begin{tabular}{|c|c|}
\hline $\begin{array}{l}\text { LEGEND: } \\
\text { (Siliodod) }\end{array}$ & = In compliance with all applicable documentation \\
\hline $\mathrm{O} / \mathrm{C}$ & = Out of compllance with documentation \\
\hline-357 & $\begin{aligned}= & \text { WHC-SD-WM-TI-357. "Waste Storage Tank Status and Leak } \\
& \text { Detection Criteria" }\end{aligned}$ \\
\hline M.T. & = Manual Tape \\
\hline FIC & $=$ Food Instrument Company \\
\hline OSRSAR & $\begin{aligned}= & \text { Operations Safety Requirements/Safoty Analysis Report } \\
& \text { Requirements }\end{aligned}$ \\
\hline N/A & $=$ Nol Applicable (l.e., no M.T., FIC installed) \\
\hline O/S & $=$ Out of Service \\
\hline W.F. & $=$ Weight Factor \\
\hline Rad. & = Radiation \\
\hline
\end{tabular}

Information as of $1 / 31 / 93$

\begin{tabular}{|c|c|c|c|c|c|c|c|}
\hline \multirow{3}{*}{$\begin{array}{l}\text { Tank } \\
\text { Number }\end{array}$} & \multirow[b]{3}{*}{ Watch List } & \multirow{3}{*}{$\begin{array}{c}\text { Temperature } \\
\text { Readings } \\
(4) \\
\text { (OSR/SAR) } \\
\end{array}$} & \multirow{2}{*}{\multicolumn{2}{|c|}{$\begin{array}{l}\text { Surface Level } \\
\text { Readings (1) } \\
(-357, \text { OSR/SAR) }\end{array}$}} & \multicolumn{3}{|c|}{ Radiation Readings } \\
\hline & & & & & \multicolumn{2}{|c|}{$\begin{array}{l}\text { Leak Detection } \\
\text { Pits (5) } \\
(-357, \text { OSR/SAR) }\end{array}$} & \multirow{2}{*}{$\begin{array}{c}\text { Annulus } \\
(-357)\end{array}$} \\
\hline & & & M.T. & FIC & W.F. & Rad. & \\
\hline 101-AN & & ? & NA & 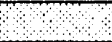 & & & \\
\hline 102-AN & & & NA & & & O/C & \\
\hline $103-\mathrm{AN}$ & $\bar{x}$ & & NIA & 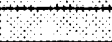 & 5 & & \\
\hline 104-AN & $x$ & & NIA & & & $\mathrm{O} / \mathrm{C}$ & 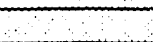 \\
\hline 105-AN & $\bar{x}$ & & N/A & $\mathrm{nz}$ & & 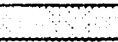 & \\
\hline 106-AN & & & N/A & 8 & 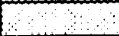 & 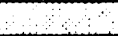 & $\ldots$ \\
\hline $107-\mathrm{AN}$ & & & N/A & $\pi$ & 3 & +2 & $\cdots$ \\
\hline 101-AP & & & O/S & 8 & & & + \\
\hline \multicolumn{8}{|l|}{ 102-AP } \\
\hline 103-AP & & & $0 / 5$ & 3 & & & \\
\hline 104-AP & & 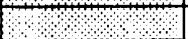 & o/s & & & & \\
\hline 105-AP & & $0 / 5$ & & & 3 & & \\
\hline $106-A P$ & & o/s & & & r & & \\
\hline 107-AP & & d/s & & मी & & & \\
\hline 108-AP & & O/S & 8 & & 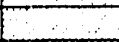 & & \\
\hline $101-A W$ & $\bar{x}$ & । & $1 \%$ & $0 / 5$ & 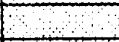 & $\mathrm{CH}$ & \\
\hline \multicolumn{8}{|l|}{ 102-AW } \\
\hline $103-A W$ & & & म & $0 / 5$ & 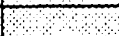 & & \\
\hline \multicolumn{8}{|l|}{ 104-AW } \\
\hline \multicolumn{8}{|l|}{ 105-AW } \\
\hline \multicolumn{8}{|l|}{ 106-AW } \\
\hline 101-AY & & & २० & O/S & & & $\mathrm{O} / \mathrm{C}$ \\
\hline $102-A Y$ & & & & & & पP & $\mathrm{O} / \mathrm{C}$ \\
\hline $101-A Z$ & & & & O/S & & & O/C \\
\hline $102-A Z$ & & & & $0 / 5$ & ? & 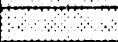 & O/C \\
\hline $101-S Y$ & $\bar{x}$ & 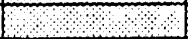 & 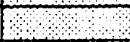 & 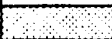 & - & 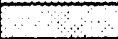 & $\ldots$ \\
\hline $102-S Y$ & & & & O/S & & & \\
\hline $103-S Y$ & $x$ & 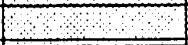 & 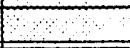 & 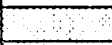 & $x^{2}$ & O/C & \\
\hline $\begin{array}{l}\text { Totals: } \\
28 \text { tanks }\end{array}$ & $\begin{array}{c}6 \\
\text { Watch List Tanks }\end{array}$ & $\begin{array}{l}\text { O/C: } \\
0(4)\end{array}$ & $\begin{array}{l}\text { O/C: } \\
0\end{array}$ & $\begin{array}{l}\text { O/C: } \\
0\end{array}$ & $\begin{array}{l}\text { O/C: } \\
0\end{array}$ & $\begin{array}{l}\text { O/C: } \\
3 \text { tanks }\end{array}$ & $\begin{array}{l}O / C: \\
4 \text { tanks }\end{array}$ \\
\hline
\end{tabular}

(1) All DSTs have both manual tape and FIC, with the exception of the AN Tank Farm which only has FICs. The manual tape is used when the FIC is out of service. O/C will be shown when no readings are obtained.

(2) Psychrometric readings are taken on DSTs. All DSTs are equipped with active exhausters. Psychrometrics were not taken from 9/91 to 9/92; readings on some tanks were resumed beginning October 1992. Frequency of psychrometric monitoring is not specified in -357 or operating procedures.

(3) In-tank photographs are not required by -357. Last in-tank photographs in DSTs were taken April 1989.

(4) DST temperature tree readings are taken weekly, with the exception of 101-SY which are obtained shiftwise with increased readings taken prior to and following gas venting.

(5) Failure of both leak detection systems requires repair of at least one system within 5 working days. Failure of one stem only, repair must be within 10 working days. Per -357 . If repair of out-of-service systems exceeds these timeframes, systems are O/C. Out-of-service systems which have not exceeded these timeframes will be shown as O/S. 


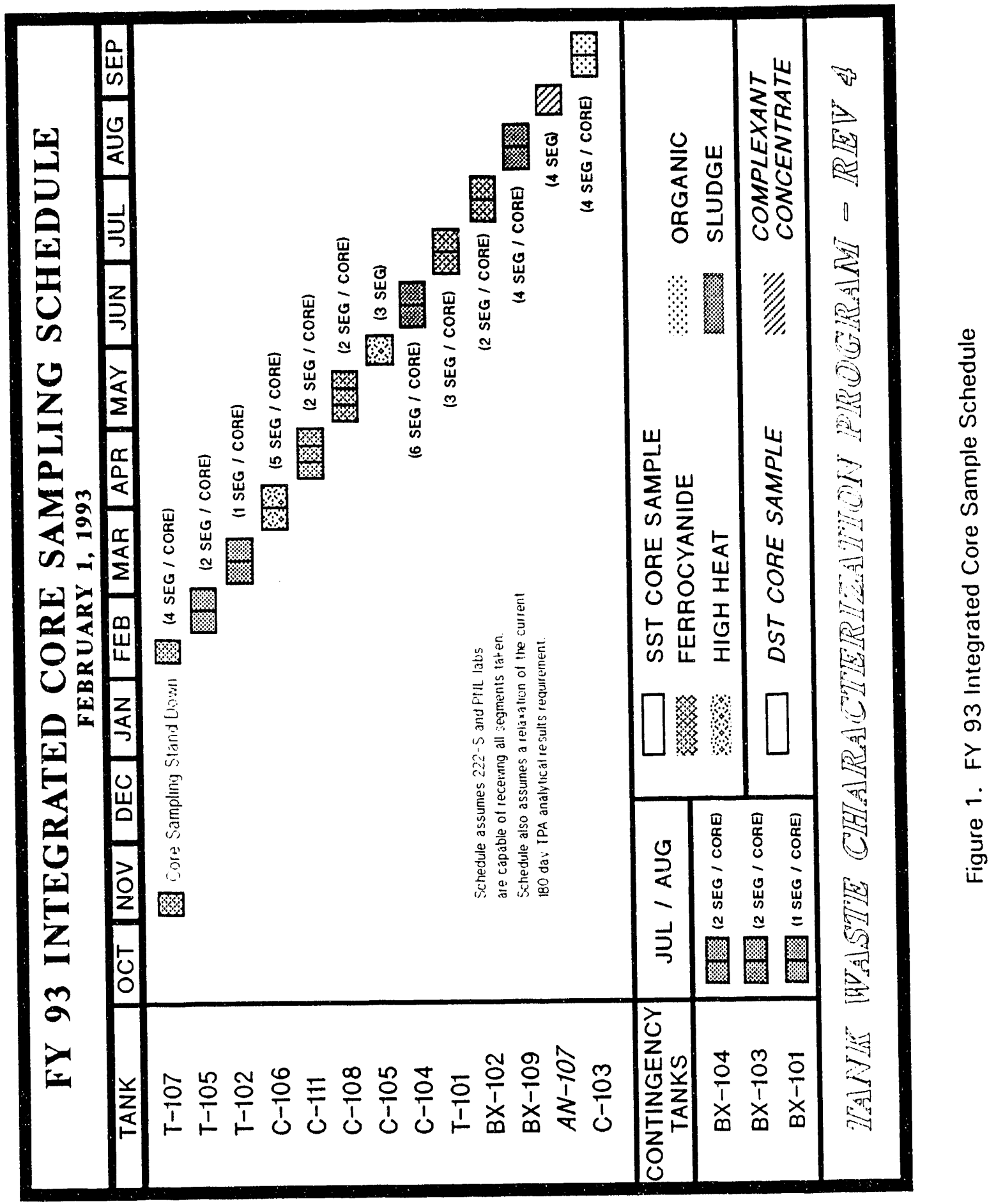




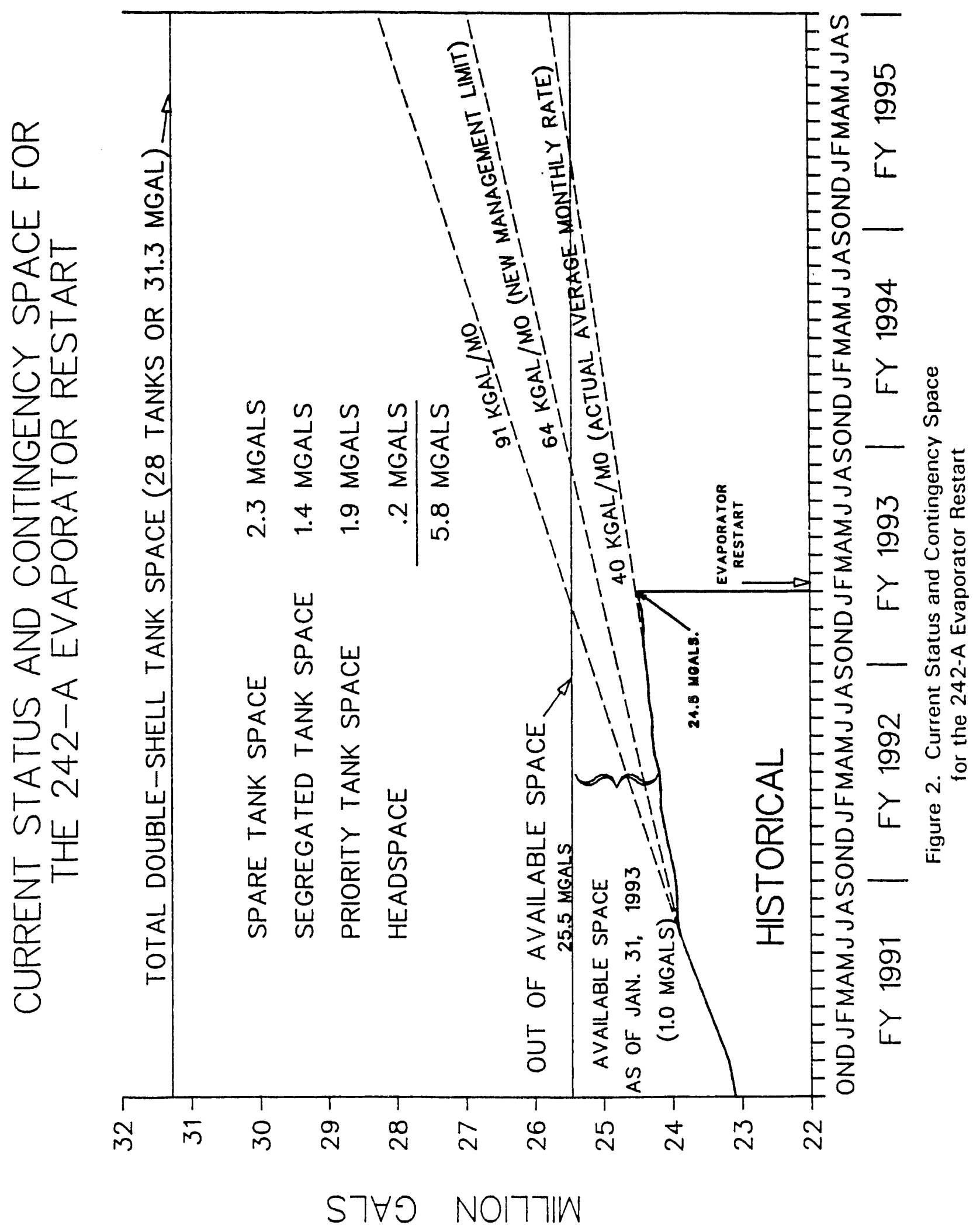




\section{Sound Single Shell Tanks}

Waste Volumes - Grouped By Waste Type

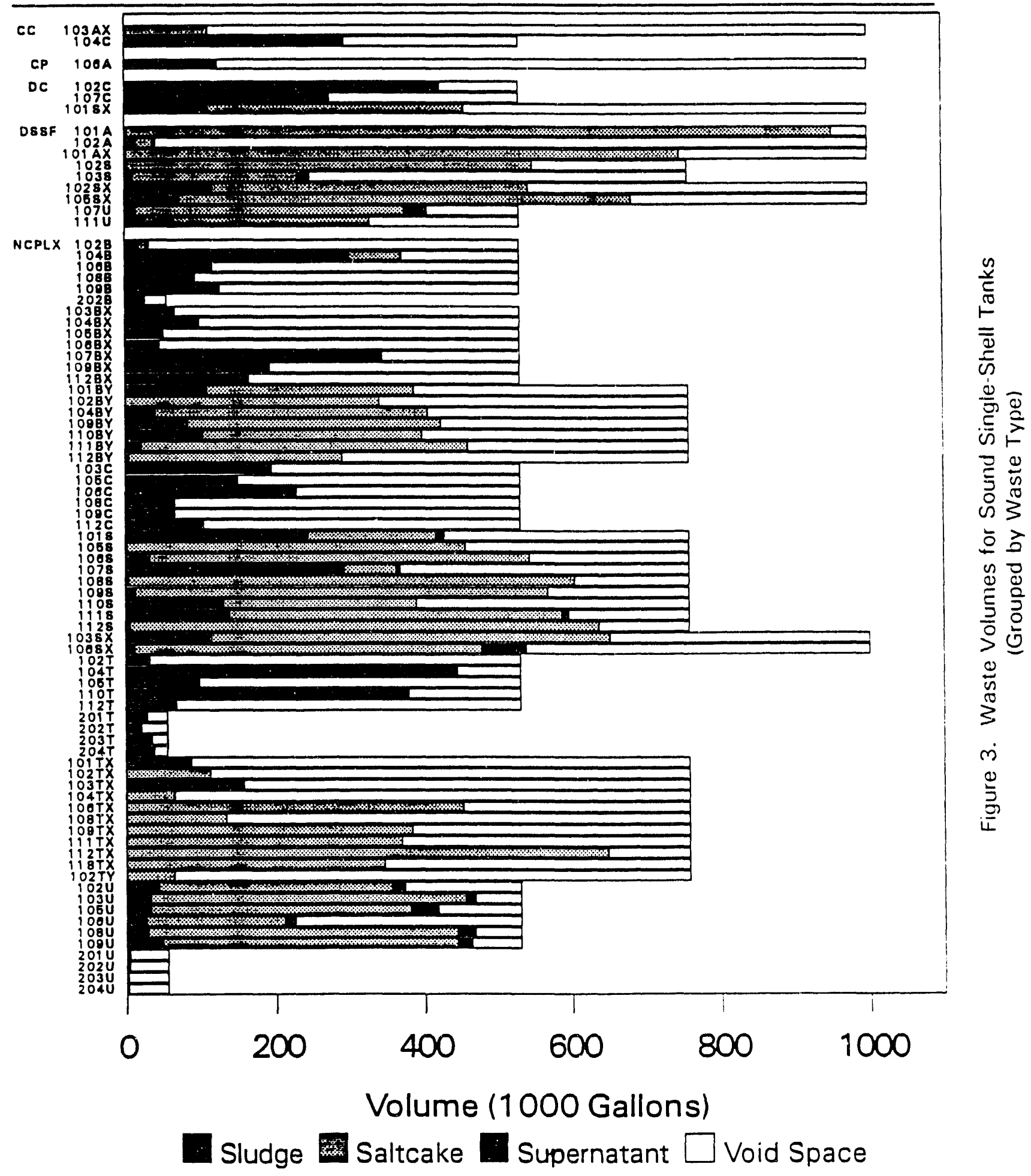




\section{Assumed Leaking Single Shell Tanks \\ Waste Volumes - Grouped By Waste Type}

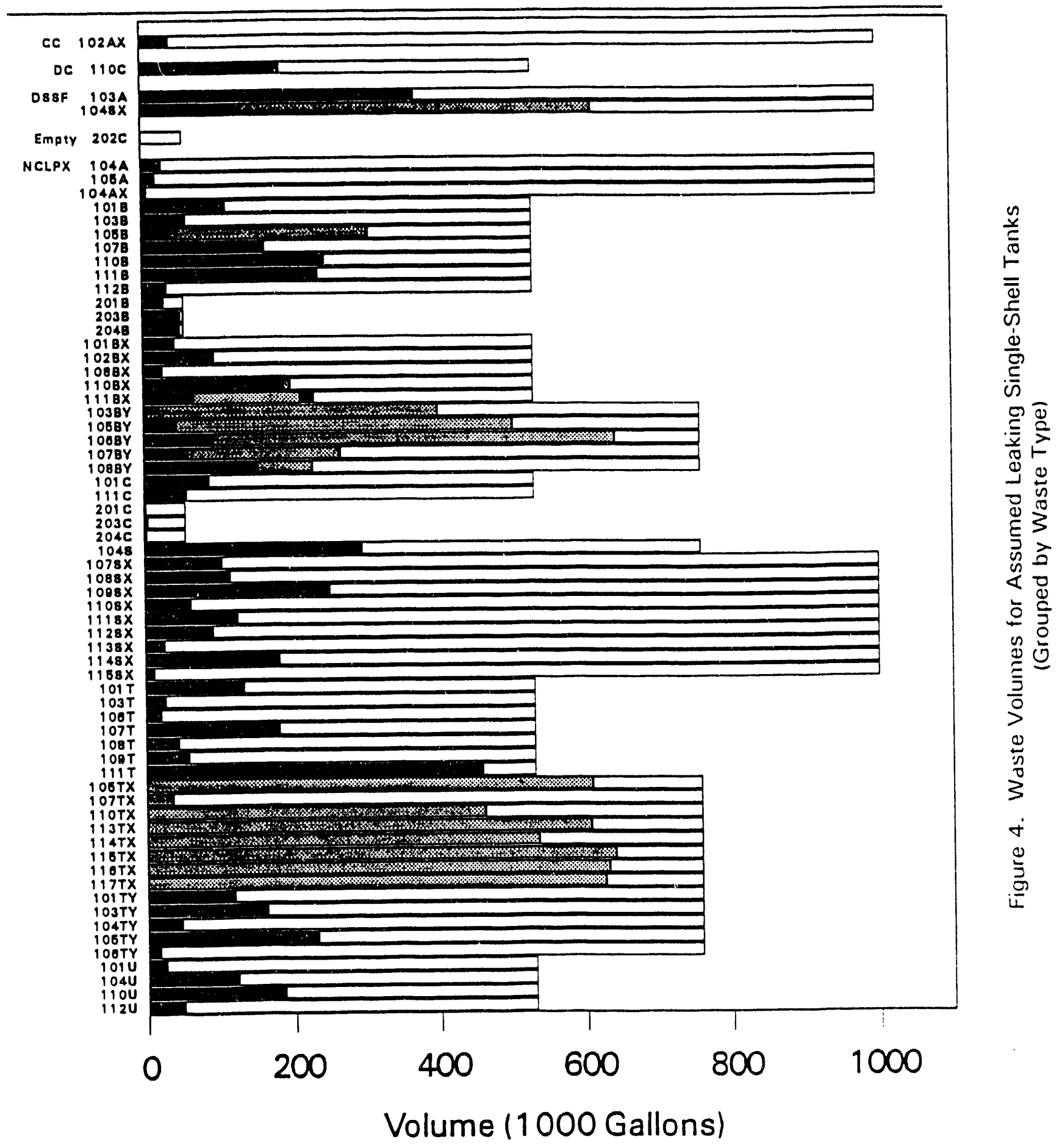

Sludge Saltcake $\square$ Supernatant $\square$ Void Space 
WHC-EP-0182-58

APPENDIX A

TANK AND EQUIPMENT CODE AND STATUS DEFINITIONS 
WHC-EP-0182-58

This page intentionally left blank 
WHC-EP-0182-58

TANK AND EQUIPMENT CODE/STATUS DEFINITIONS

January 1993

\section{IANK STATUS CODES}

\section{WASTE TYPE}

$\begin{array}{ll}\text { AGING } & \text { Aging Waste (Neutralized Current Acid Waste [NCAW]) } \\ \text { CC } & \text { Complexant Concentrate Waste } \\ \text { CP } & \text { Concentrated Phosphate Waste } \\ \text { DC } & \text { Dilute Complexed Waste } \\ \text { DN } & \text { Dilute Non-Complexed Waste } \\ \text { DSS } & \text { Double-Shell Slurry } \\ \text { DSSF } & \text { Double-Shell Slurry Feed } \\ \text { NCPLX } & \text { Non-Complexed Waste } \\ \text { PD/PN } & \text { Plutonium-Uranium Extraction (PUREX) Neutralized Cladding } \\ & \text { Removal Waste (NCRW) transuranic waste (TRU) } \\ \text { PT } & \text { Plutonium Finishing Plant (PFP) TRU Solids }\end{array}$

TANK USE (DOUBLE-SHELL TANKS ONLY)

CWHT Concentrated Waste Holding Tank

DRCVR Dilute Receiver Tank

EVFD Evaporate Feed Tank

GRTFD Grout Feed Tank

SRCVR Slurry Receiver Tank

\section{SOLID AND LIQUID VOLUME DETERMINATION METHODS}

$\begin{array}{ll}F & \text { Food Instrument Company (FIC) Automatic Surface Level Gauge } \\ M & \text { Manual Tape Surface Level Gauge } \\ P & \text { Photo Evaluation } \\ S & \text { Sludge Level Measurement Device }\end{array}$

\section{DEFINITIONS}

\section{WASTE TANKS - GENERAL}

$\begin{array}{ll}\text { Waste Tank } & \begin{array}{l}\text { A potentially unsafe condition in the handling of } \\ \text { Safety Issue }\end{array} \\ \text { waste material in underground storage tanks that } \\ \text { requires corrective action to reduce or eliminate } \\ \text { the unsafe condition }\end{array}$


WASTE TYPES

Aging Waste
(AGING)

Concentrated Complexant (CC)

Concentrated Phosphate Waste $(C P)$

Dilute Complexed Waste (DC)
High level, first cycle solvent extraction waste from the PUREX plant (NCAW)

Concentrated product from the evaporation of dilute complexed waste.

Waste originating from the decontamination of $100 \mathrm{~N}$ Area reactor. Concentration of this waste produces concentrated phosphate waste.

Characterized by a high content of organic carbon including organic complexants:

ethylenediaminetetra-acetic acid (EDTA), citric acid, hydroxyethyl-ethylenediaminetriacetic acid (HEDTA), and iminodiacetate (IDA) being the major complexants used. Main sources of DC waste in the DST system are saltwell liquid inventory.

Dilute Non-Complexed Waste (DN)

Low activity liquid waste originating from $T$ and $S$ Plants, the 300 and 400 Areas, PUREX facility (decladding supernatant and miscellaneous wastes), $100 \mathrm{~N}$ Area (sulfate waste), B Plant, saltwells, and PFP (supernate).

Double-Shell

Slurry (DSS)

Waste that exceeds the sodium aluminate saturation boundary in the evaporator without exceeding receiver tank composition limits. For reporting purposes, DSS is considered a solid.

Double-Shell Slurry Feed (DSSF)

Waste concentrated just before reaching the sodium aluminate saturation boundary in the evaporator without exceeding receiver tank composition limits. This form is not as concentrated as DSS.

Non-complexed (NCPLX)

General waste term applied to all Hanford site liquors not identified as complexed.

PUREX Decladding (PD/PN)

PUREX Neutralized Cladding Removal Waste (NCRW) is the solids portion of the PUREX plant neutralized cladding removal waste stream; received in Tank Farms as a slurry. NCRW solids are classified as transuranic (TRU) waste.

PFP TRU Solids (PT)

TRU solids fraction from PFP Plant operations.

Drainable Interstitial Liquid (OIL)

Interstitial liquid that is not held in place by capillary forces, and will therefore migrate or move by gravity.

Supernate

The liquid above the solids in waste storage tanks. 
Ferrocyanide

WASTE STATUS

In-Service Tank

Out-of-Service

Tank
A compound of iron and cyanide commonly expressed as FeCN. The actual formula for the ferrocyanide anion is $\left[\mathrm{Fe}(\mathrm{CN})_{6}\right]^{-4}$.

The waste classification of a tank being used, or planned for use, for the storage of liquid (in excess of a minimum supernatant liquid heel) in conjunction with production and/or waste processing.

A tank which does not meet the definition of an inservice tank. Before September 1988, these tanks ivere defined as inactive in this report. [Note: All single-shell tanks (SST) are out of service.]

\section{STABILIZATION (Single-Shell Tanks only)}

Interim

Stabilized

(IS)
A tank which contains less than 50,000 gal of drainable interstitial liquid and less than 5,000 gal of supernatant liquid. If the tank was jet pumped to achieve interim stabilization, then the jet pump flow must also have been at or below $0.05 \mathrm{gpm}$ before interim stabilization criteria is met.

\section{ISOLATION (Single-Shell Tanks only)}

Partially

Interim Isolated

(PI)

Interim Isolated (II)

\section{TANK INTEGRITY}

Sound

Assumed Leaker
The administrative designation reflecting the completion of the physical effort required for Interim Isolation except for isolation of risers and piping that is required for jet pumping or for other methods of stabilization.

The administrative designation reflecting the completion of the physical effort required to minimize the addition of liquids into an inactive storage tank, process vault, sump, catch tank, or diversion box.

The integrity classification of a waste storage tank for which surveillance data indicate no loss of

liquid attributed to a breach of integrity.

The integrity classification of a waste storage tank for which surveillance data indicate a loss of liquid attributed to a breach of integrity.

\section{TANK INVESTIGATION}

Intrusion
A term used to describe the infiltration of liquid into a waste tank. 


\section{SURVEILLANCE INSTRUMENTATION}

Drywe11s

Laterals

Surface Levels

Automatic FIC

Annulius
Drywells are vertical boreholes with 6-in. (internal diameter) carbon steel casings positioned radially around SSTs. Periodic monitoring is done by gamma radiation or neutron sensors to obtain scan profiles of radiation or moisture in the soil as a function of well depth, which could be indicative of tank leakage. Those wells range between 50 and $250 \mathrm{ft}$ in depth, and are monitored between the range of 50 to $150 \mathrm{ft}$. The wells are sealed when not in use. They are called drywells because they do not penetrate to the water table and are therefore usually "dry." The drywell frequency monitoring schedule calls for 105 drywells weekly, 91 biweekly, 41 monthly, 151 quarterly, and 371 annually.

Laterals are homizontal drywells positioned under single-shell waste storage tanks to detect radionuclides in the soil which could be indicative of tank leakage. These diywells are monitored by radiation detection probes. Laterals are 4 -in. inside diameter steel pipes located 8 to $10 \mathrm{ft}$ below the tank's concrete base. There are three laterals per tank. Laterais are located only in $A$ and $S X$ farms.

The surface level measurements in all waste stcrage tanks are monitored by manual or automatic conductivity probes, and recorded and transmitted or inputted to the Computer Automated Surveillance System (CASS).

An automatic waste surface level measurement device is manufactured by the Food Instrument Company (FIC). The instrument consists of a conductivity electrode (plummet) connected to a calibrated steel tape, a steel tape reel housiny aid a controller that automatically raises and lowers the plummet to obtain a waste surface level reading. The controller can provide a digital display of the data and a 1. "ransmit the reading to the CASS. Some tanks have gauges zonnected to CASS and others are read manualiy.

The annulus is the space between the inner and outer shells on DSTs. Drain channels in the insulating and/or supporting concrete carry any leakage to the annulus space where conductivity probes are installed. Alarms from the annunciators are received by CASS. Continuous Air Monitoring (CAM) alarms are also located in the annulus. The annulus conductivity probes and radiation detectors are the primary means of leak detection for all DSTs. 


\section{WHC-EP-0182-58}

Liquid Observation Well ( $:$ OW)

Thermocouple (TC)

In-tank

Photography
In-tank liquid observation wells are used for monitoring the interstitial liquid level (ILL) in single-shell waste storage tanks. The wells are constructed of fiberglass or TEFZEL*-reinforced epoxy-polyester resin, sized to extend to within 1 in. of the bottom of the tank steel liner. They are sealed at their bottom ends and have a nominal outside diameter of $3.5 \mathrm{in}$. Three probes are used to monitor changes in the ILL: acoustic; gamma; and neutron, which can indicate intrusions or leakage by increases or decreases in the ILL. There are 58 LOWs (56 are in operation) installed in SSTs that contain or are capable of containing greater than $50,000 \mathrm{gal}$ of drainable interstitial liquid, and in two DSTs only. The LOWs installed in two DSTs, (102-SY and 103-AW Tanks), are constructed of steel and are used for special surveillance purposes only.

A thermocouple is a thermoelectric device used to measure temperature. More than one thermocouple on a device (probe) is called a thermocouple tree. In DSTs there may be one or more thermocouple trees in risers in the primary tank. In addition, in DSTs only, there are thermocouple elements installed in the insulating concrete, the lower primary tank knuckle, the secondary tank concrete foundation, and in the outer structural concrete. These monitor temperature gradients within the concrete walls, bottom of the tank, and the domes. In SSTs, there may be one or more thermocouple trees installed directly in a tank, although some SSTs do not have any trees installed. A single thermocouple may be installed in a riser, or lowered down an existing riser or LOW. There are also four thermocouple laterals beneath Tank 105-A in which temperature readings are taken in 34 thermocouples.

In-tank photographs may be taken to aid in resolving in-tank measurement anomalies and determine tank integrity. Photographs help determine sludge and liquid levels by visual examination.

\section{INVENTORY AND STATUS BY TANK - COLUMN CALCULATIONS (SINGLE-SHELL TANKS)}

\section{COLUMN HEADING}

Total Waste Solids Volume plus Supernatant liquid.

Supernatant Drainable Liquid Remaining minus Drainable Interstitial. Liquid Supernate is usually derived by subtracting the solids level measurement from the liquid level measurement.

* TEFZEL, a trademark of E. 1. du Pont de Nemours \& Company 
WHC-EP-0182-58

Drainable

Interstitial

Total Jet

Pumped

Drainable

Liquid

Remaining

Pumpable

Liquid

Remaining

Sludge

Saltcake

Solids Volume Update

Solids Update Source - See Footnote

Last Photo

Date

Change Since Last Monthly Report
Drainable Liquid Remaining minus Supernate.

Drainable Interstitial Liquid is calculated based on the saltcake and sludge volumes, using average porosity values or actual data for each tank, when available.

Cumulative total pumped 1979 to date.

Supernate plus Drainable Interstitial.

Drainable Liquid Remaining less undrainable heel volume.

Solids formed during sodium hydroxide additions to waste. Sludge usually was in the form of suspended solids when the waste was originally received in the tank from the waste generator. In-tank photographs may be used to estimate the volume.

Results from crystalization and precipitation after concentration of liquid waste, usually in an evaporator. If saltcake is layered over sludge, it is only possible to measure total solids volume. In-tank photographs may be used to estimate the saltcake volume.

Indicates the latest update of any change in the solids volume.

Indicates the source or basis of the latest solids volume update.

Date of latest in-tank photographs taken.

Indicates any change made since the previous month. Explanation for the change follows the Inventory and Status by Tank section. 
WHC-EP-0182-58

\section{APPENDIX B}

TANK FARM CONFIGURATION, STATUS, AND FACILITY CHARTS 
WHC-EP-0182-58

This page intentionally left blank. 

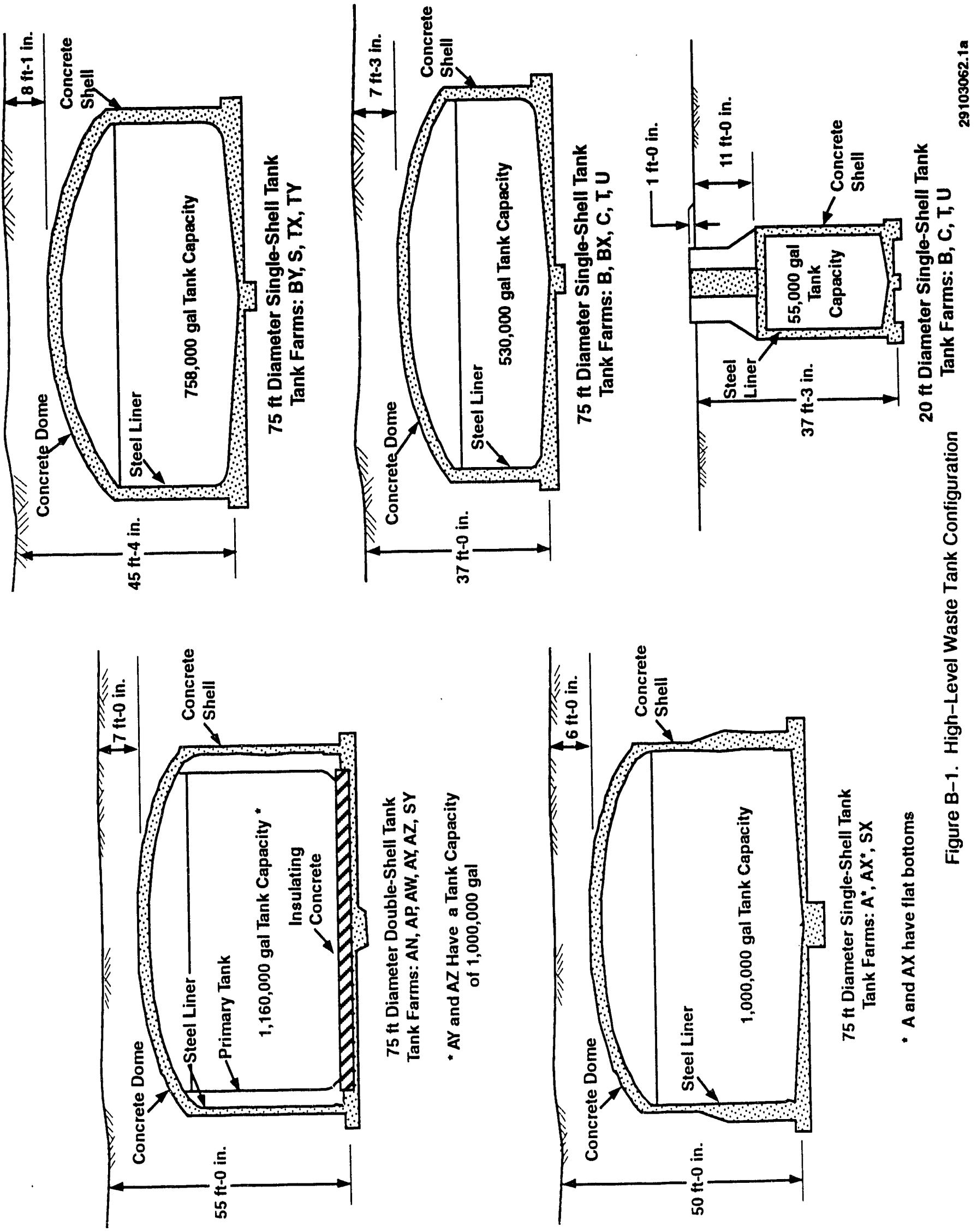

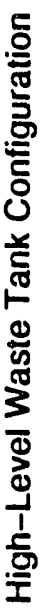




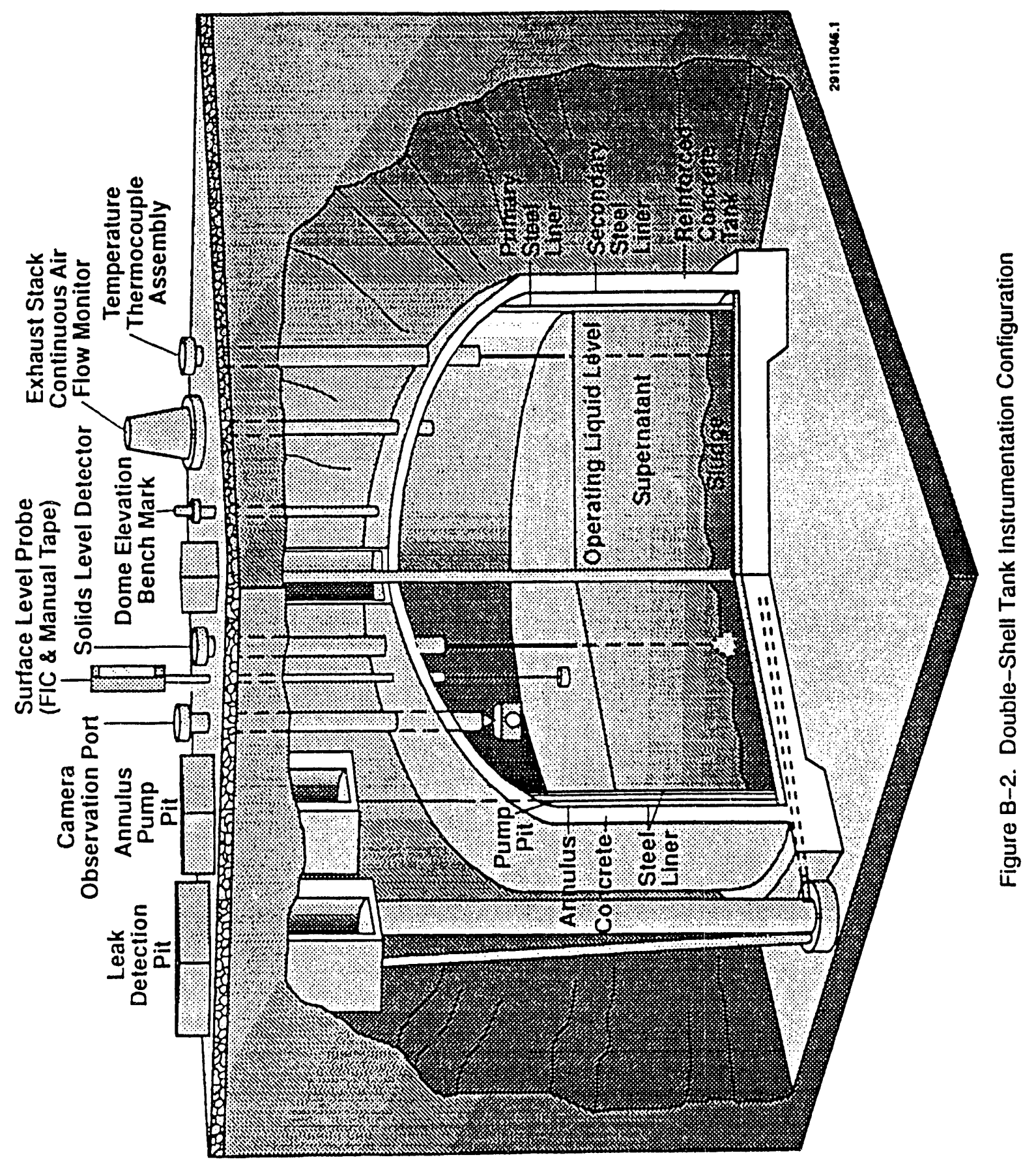




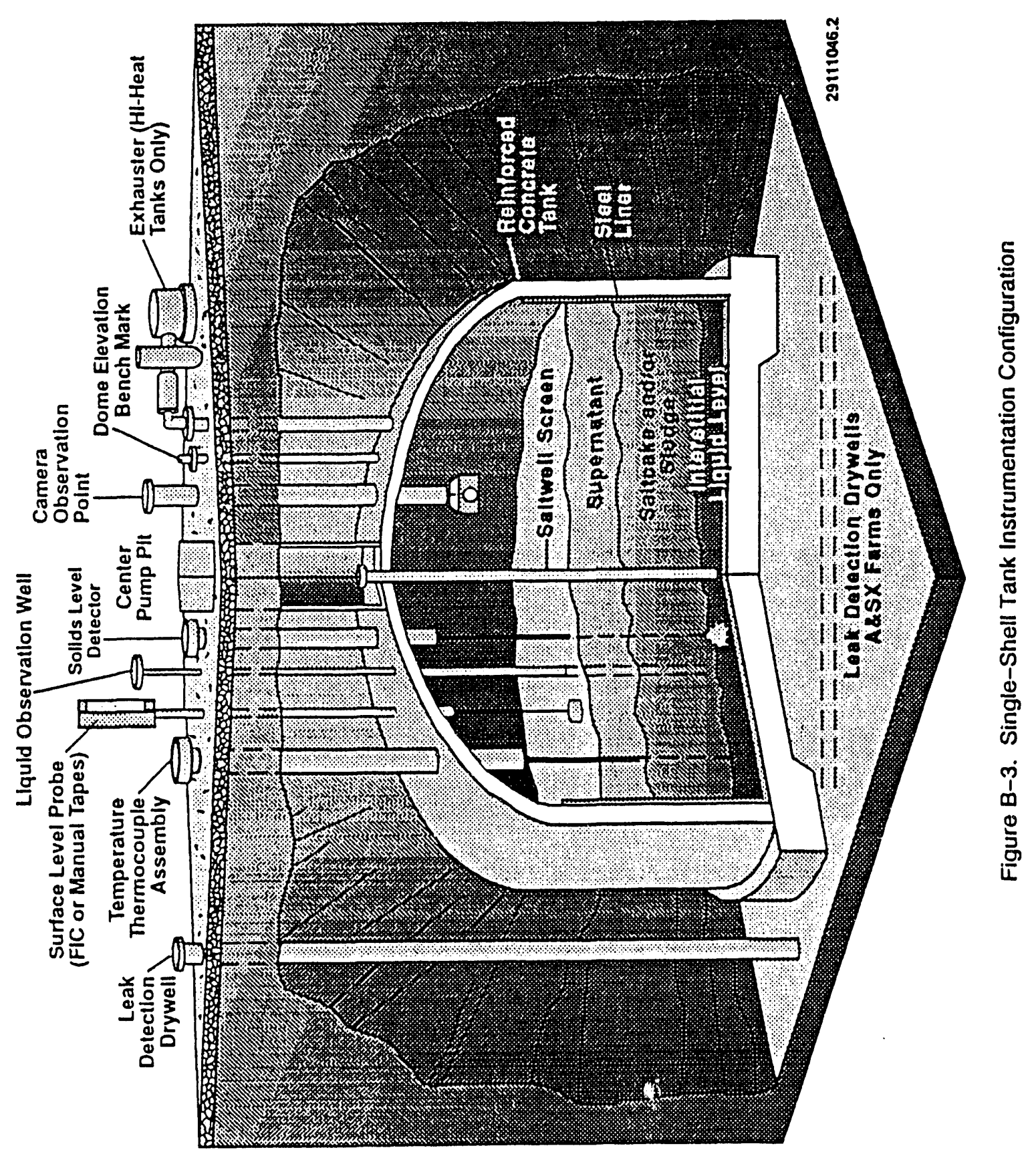


WHC-EP-0182

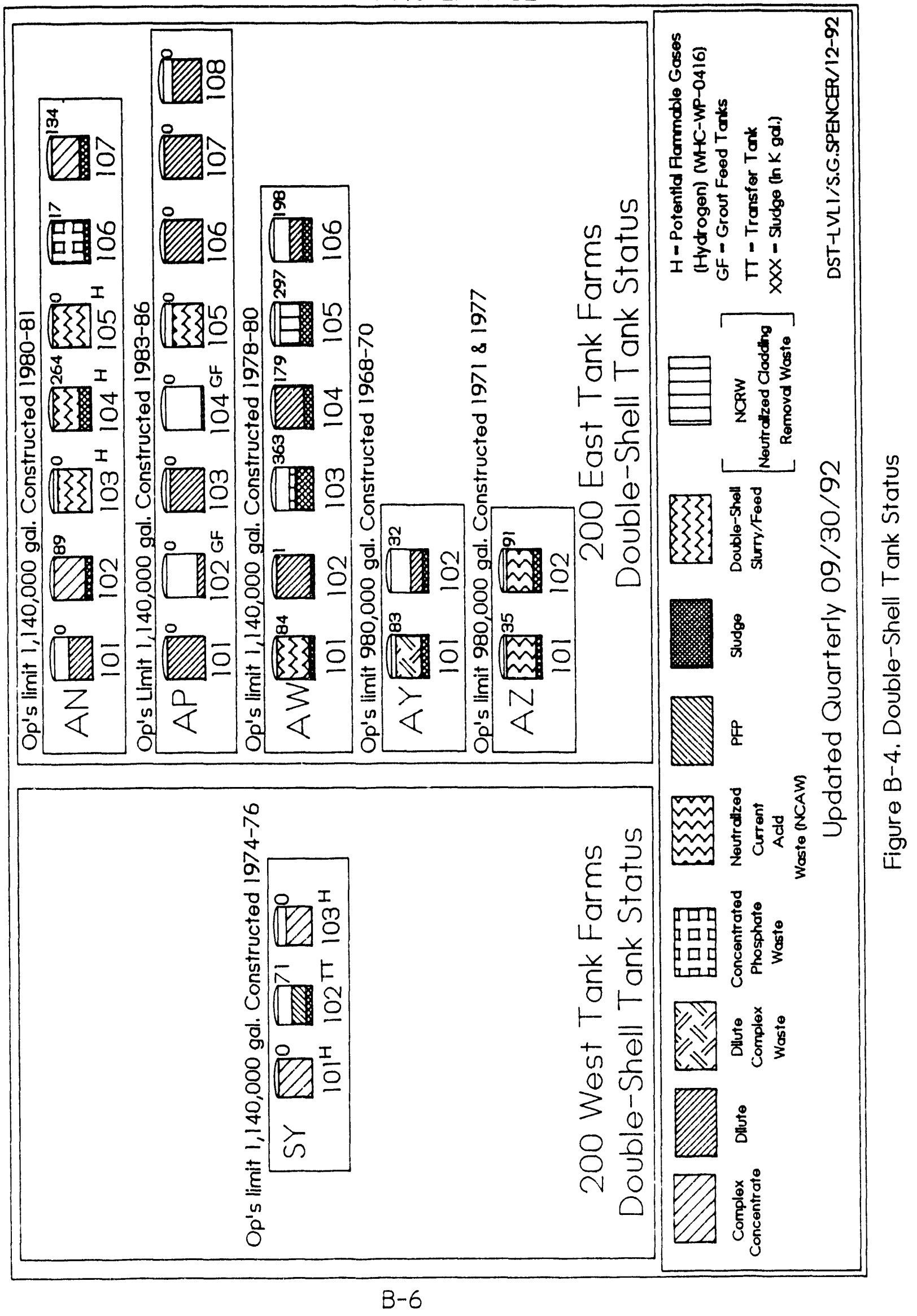


1,000,000 gal. tanks Constructed 1854-65

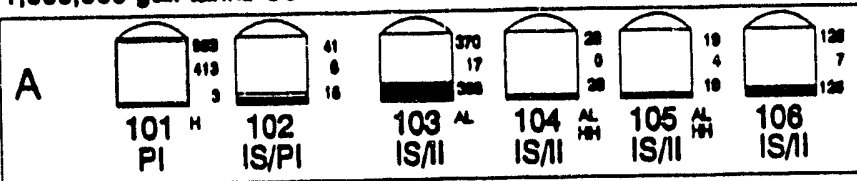

1,000,000 gal. tanks Constructed 1963-64

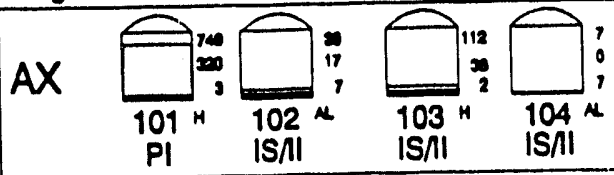

500,000 gal. tanks Constructed 1943-44

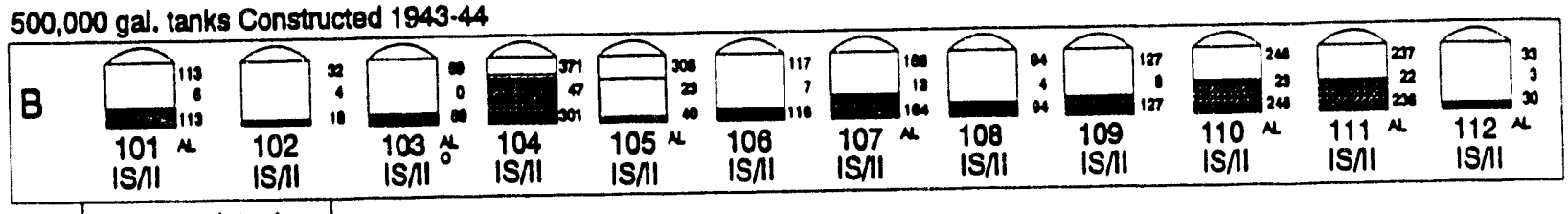

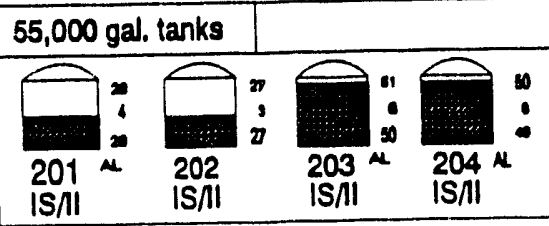

500,000 gal. tanks Constructed 1946-47

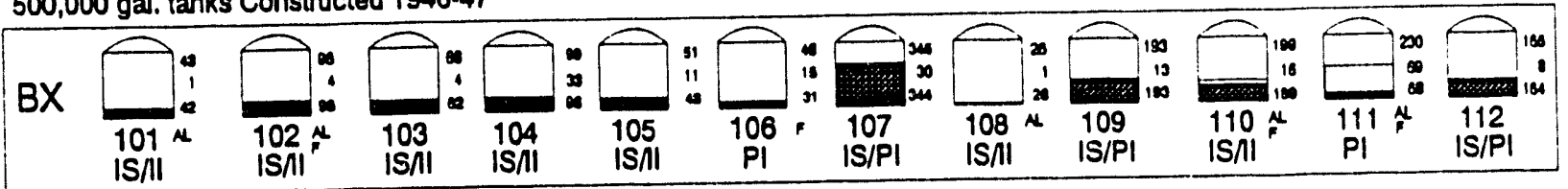

750,000 gal. tanks Constructed 1948-49

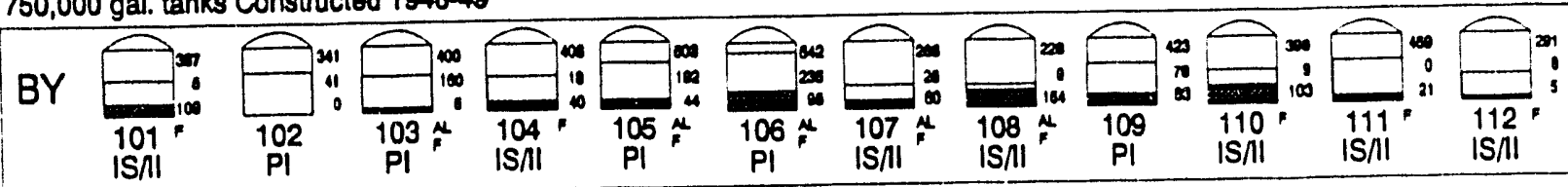

500,000 gal. tanks Constructed 1943-44
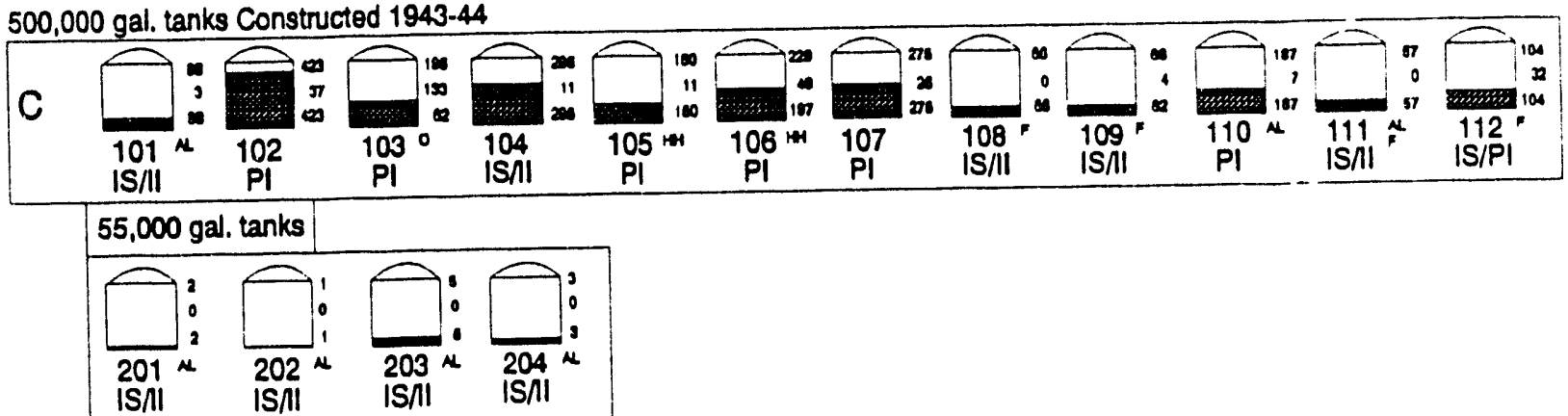

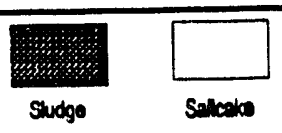

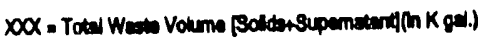

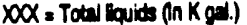

Drainable intersilel + Supomatent

xoX - sudige (in K ged )

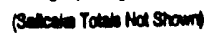

N. a Acoumed Leake

H. Hion Hin Tam

F F Forrocyeride

macerasen

$0=$ Organicas

$H=$ Potentid Fummablo Geses

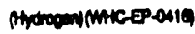

Updated Quarterly 12/31/92
II - Interim Isolaled

IS a Interim Stabilaed

$\mathrm{PI}$ a Partialy Intenm isolated

SST-ALLS.Q. SPENCEROOS-93

Figure B-5. 200E Single-Shell Tank Status 
WHC-EP-0182-58

This page intentionally left blank.

B- 8 
750,000 gal. tanks Constructed 1950-51

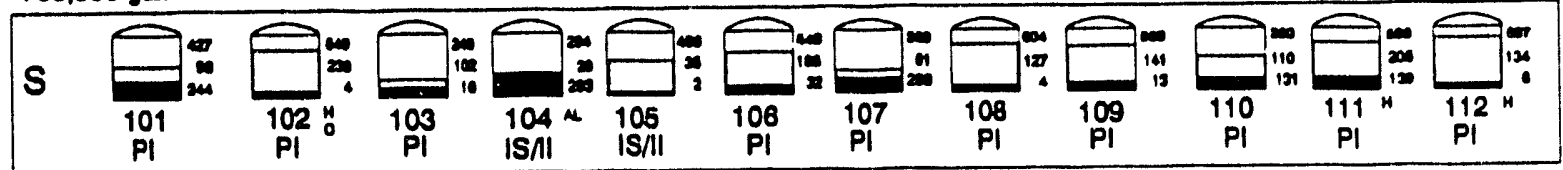

1,000,000 gal. tanks Conotructed 1953-64

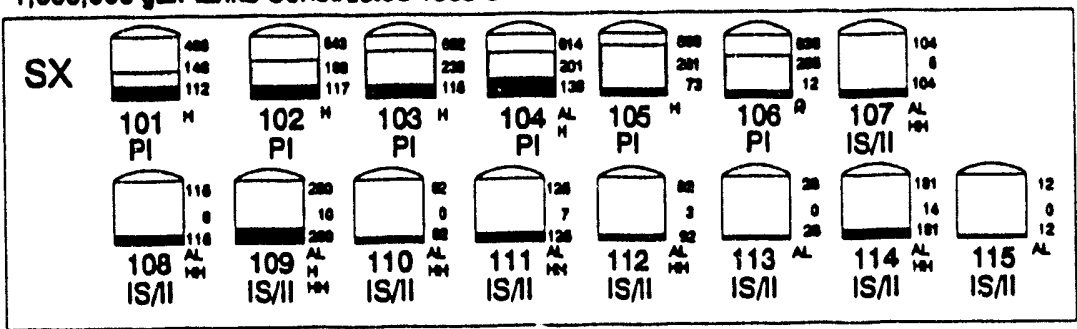

500,000 gal. tanks Constructed 1943-44

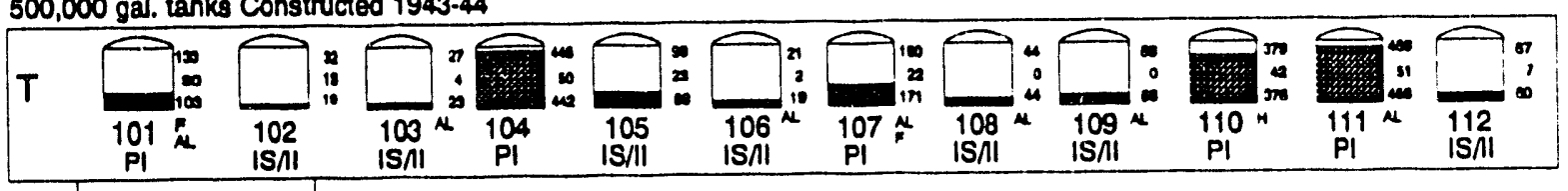

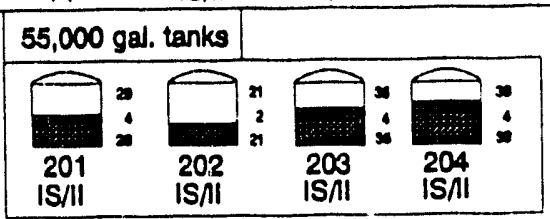

750,000 gal. tanks Constructed 1947-48

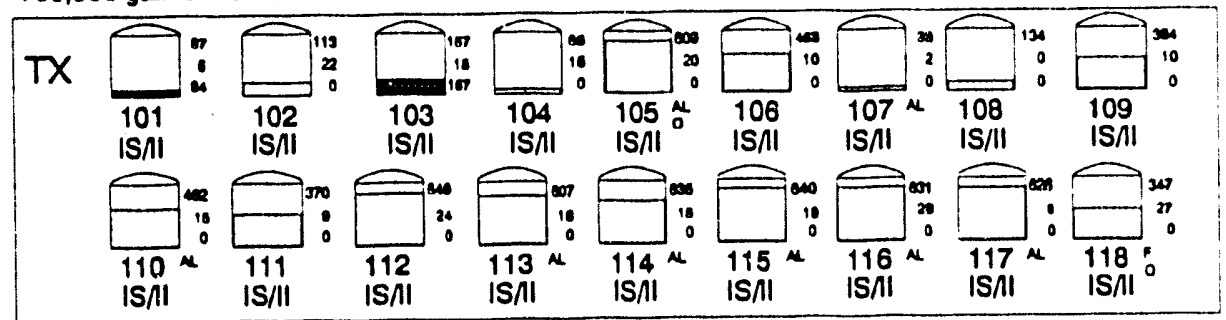

$750,000 \mathrm{gal}$. tanks Constructed 1951.52

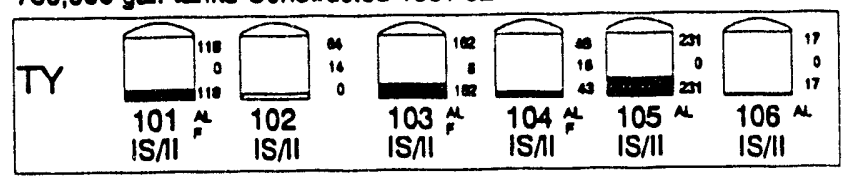

500,000 gal. tanks Constructed 1943.44

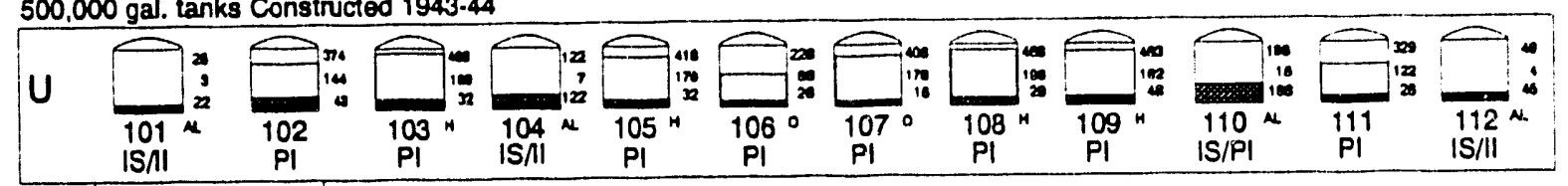

55,000 gal. tanks

\begin{tabular}{|c|c|c|c|}
\hline $\begin{array}{l}201 \\
\text { IS } / 1\end{array}$ & $\begin{array}{l}202 \\
\text { IS } 11\end{array}$ & $\begin{array}{l}203 \\
18 / 1\end{array}$ & $\begin{array}{l}204 \\
\text { IS } 11\end{array}$ \\
\hline
\end{tabular}

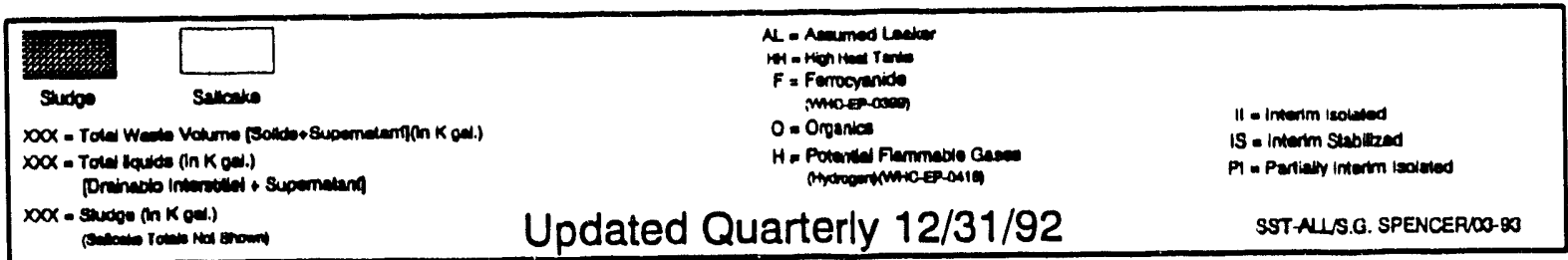

Fig. B-6. 200W Single-Shell Tank Status 
WHC-EP-0182-58

This page intentionally left blank. 
WHC-EP-0182



Figure B-7. Tank Farm Facilitios - Quick Reference

B-11/12 


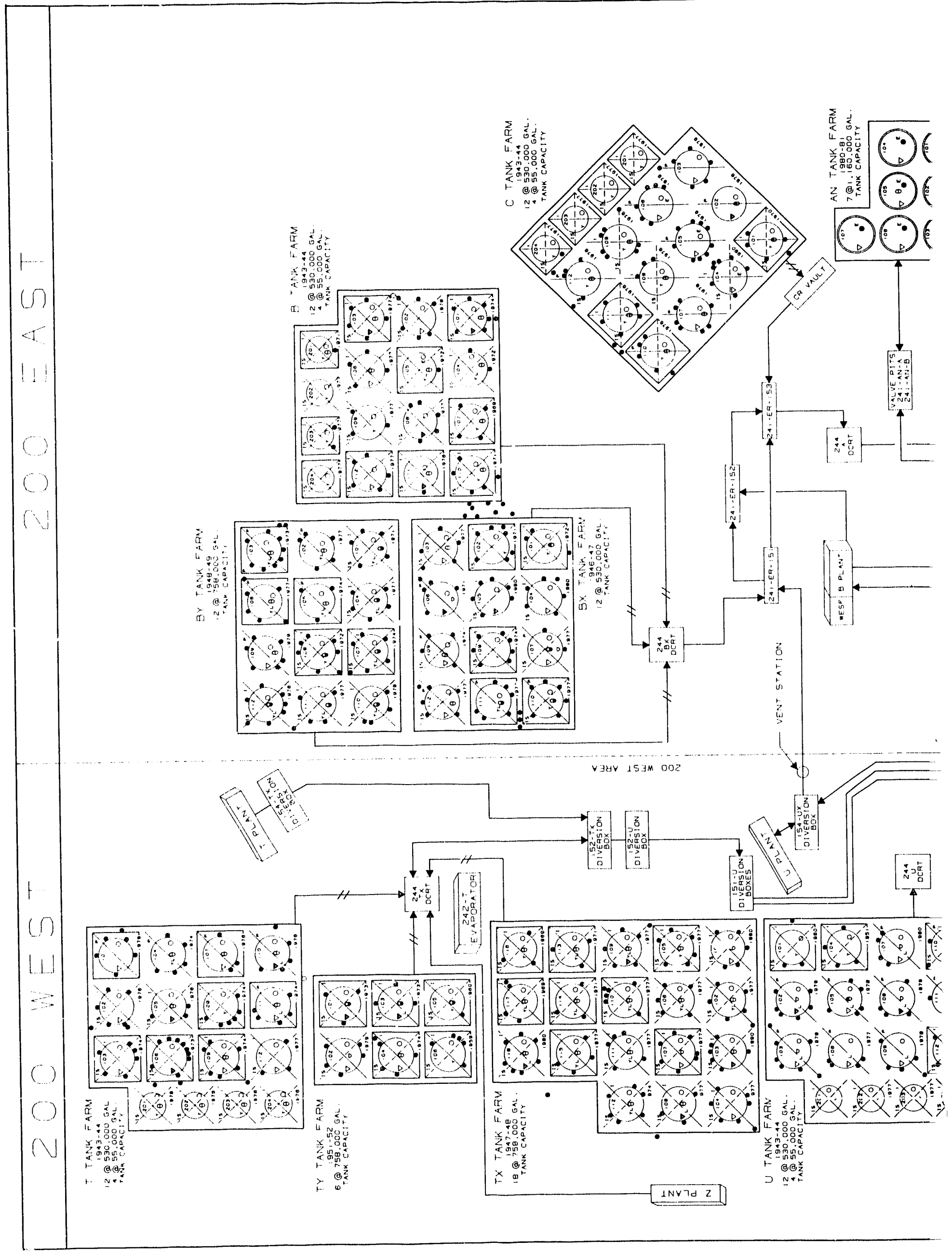




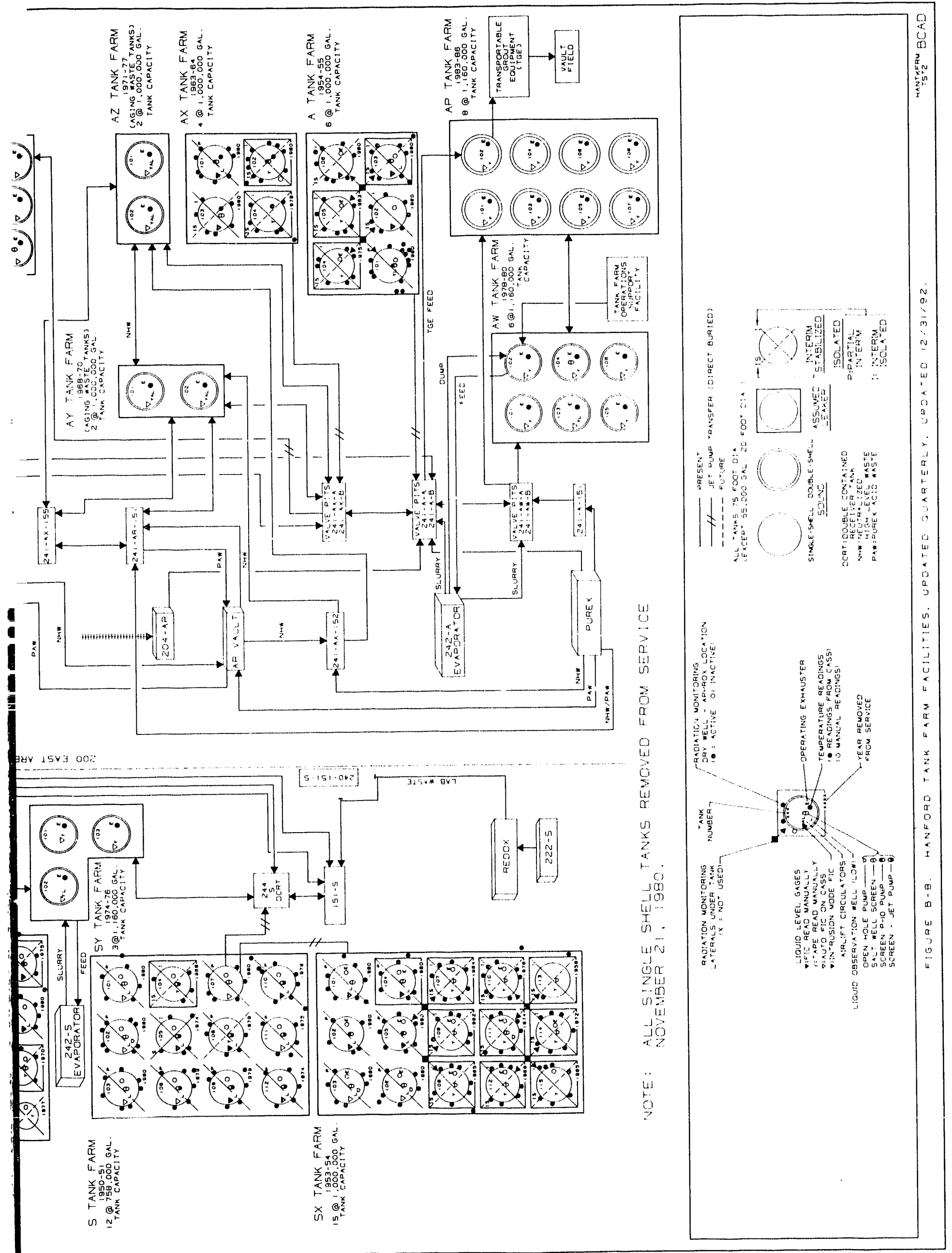


WHC-EP-0182-58

\section{APPENDIX C}

MONTHLY SUMMARY

TANK USE SUMMARY

INVENTORY SUMMARY BY TANK FARM INVENTORY AND STATUS BY TANK 
WHC-EP-0182-58

This page intentionally left blank. 







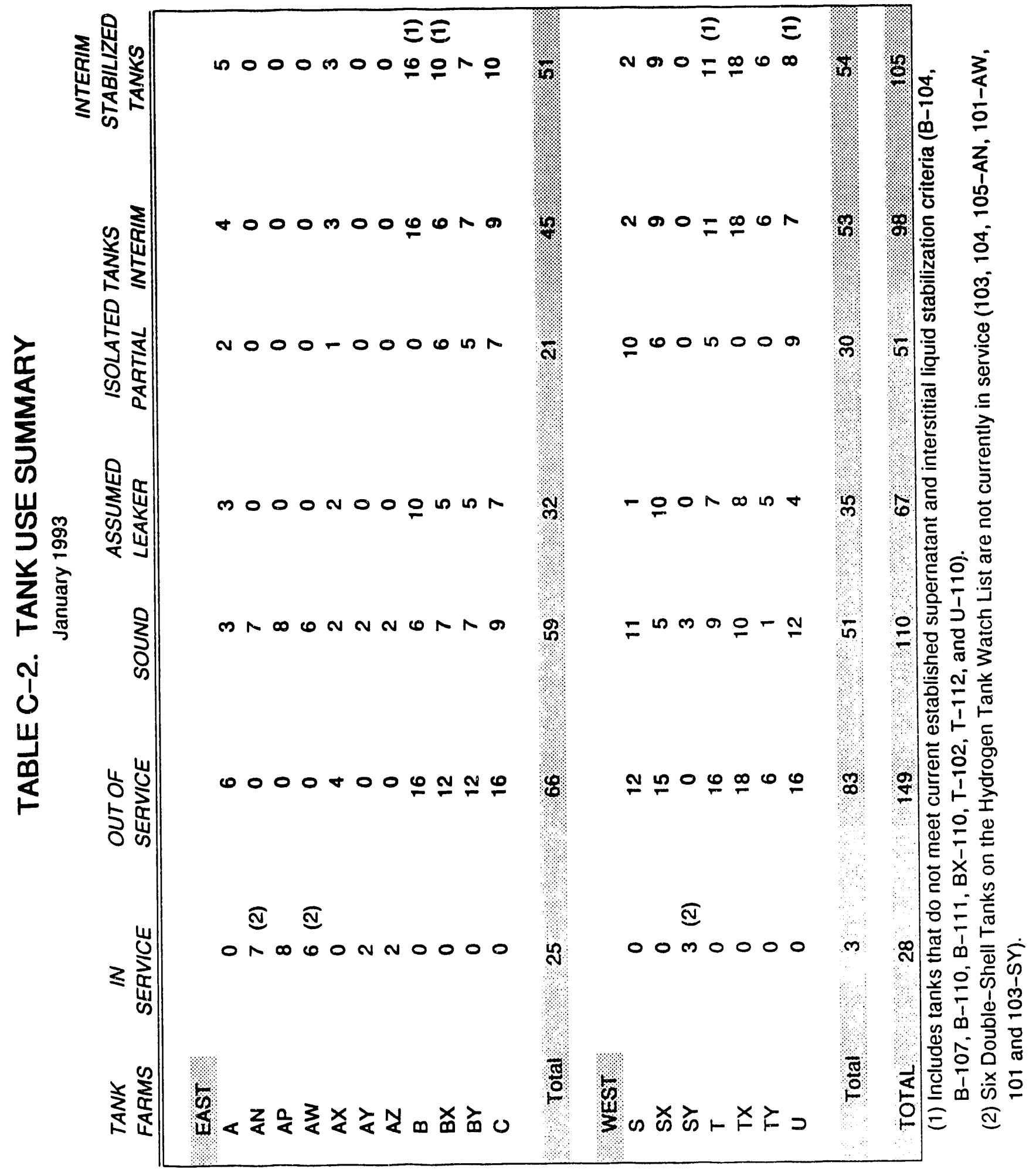




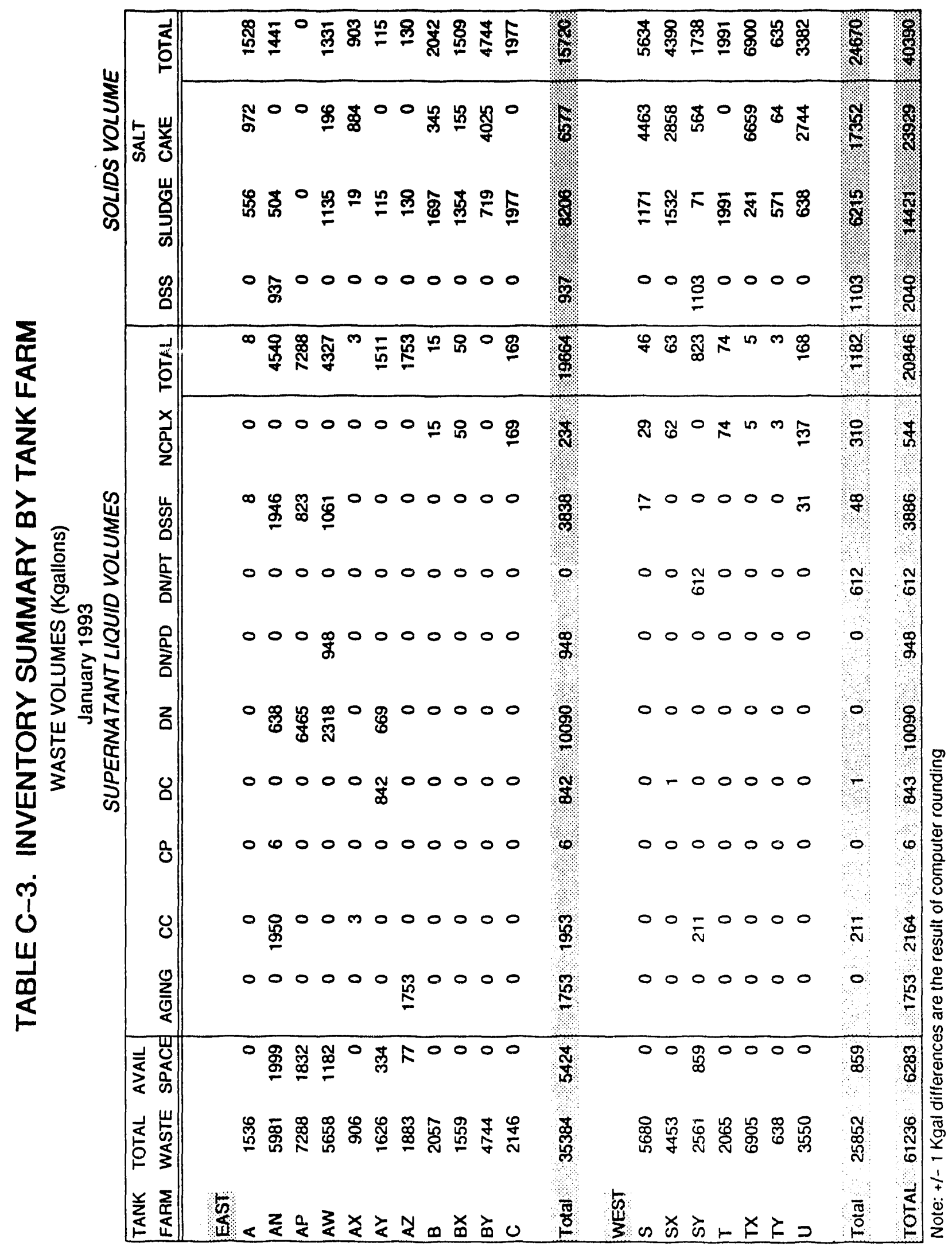




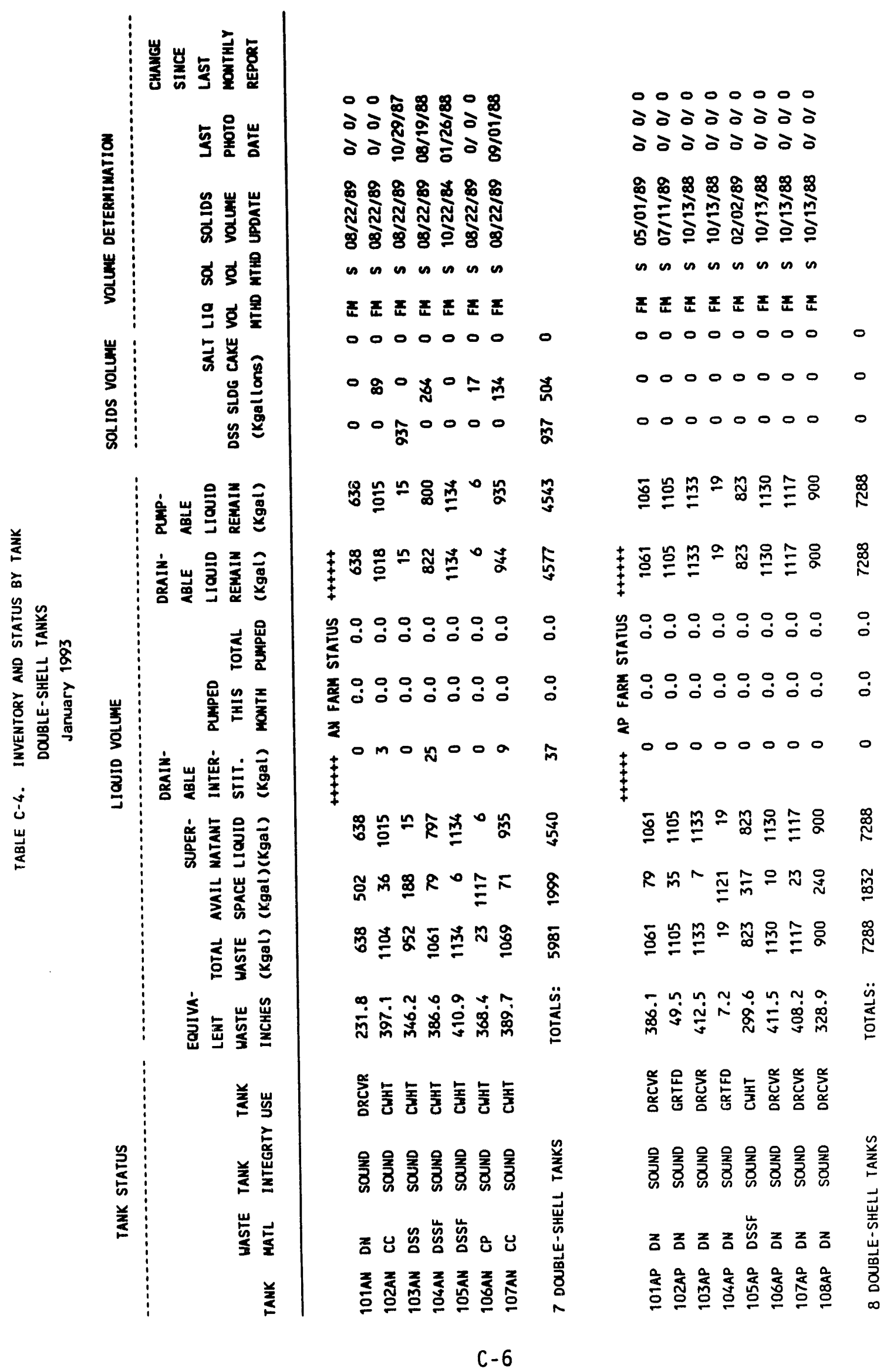




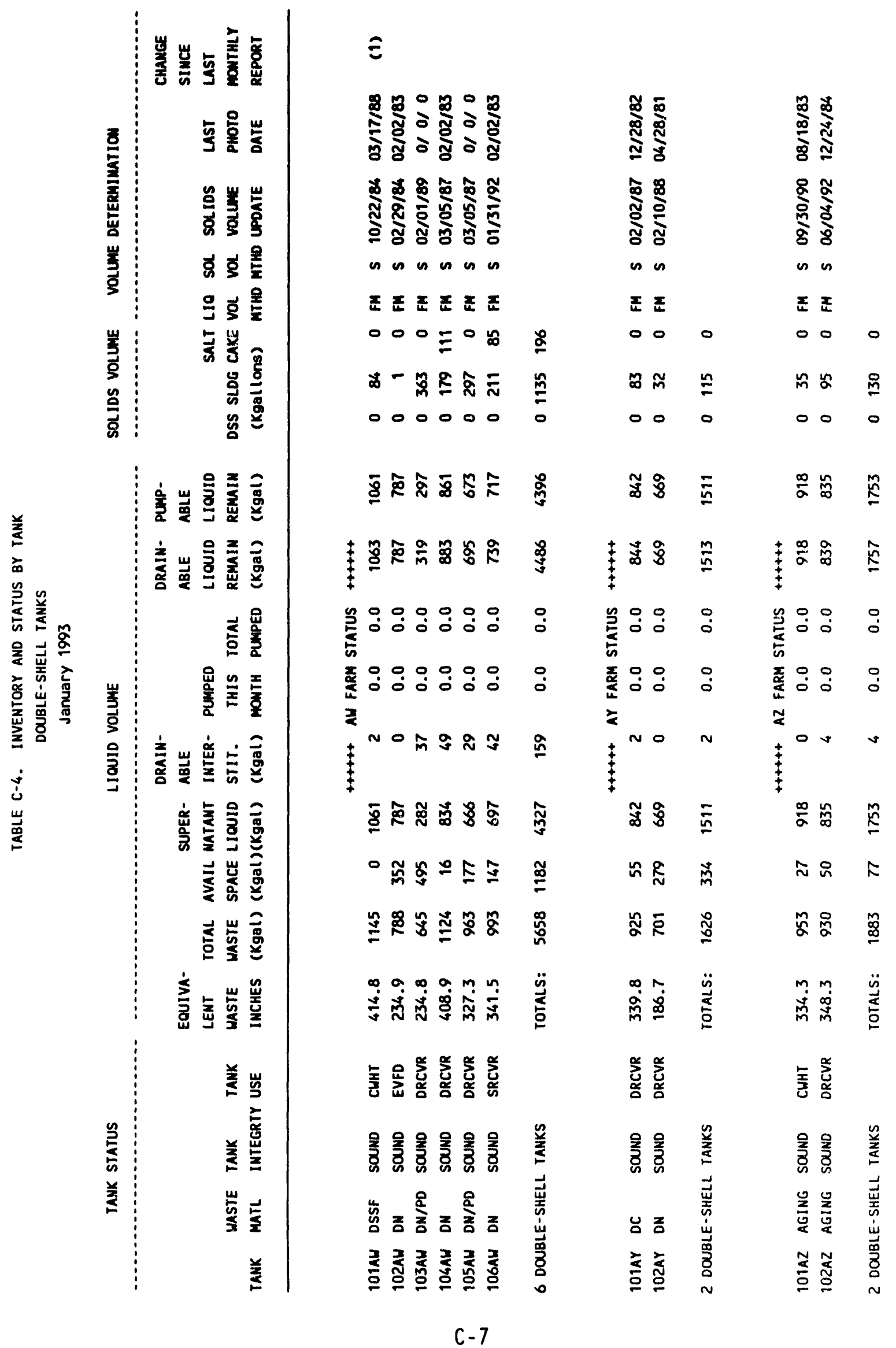


WHC-EP-0182-58

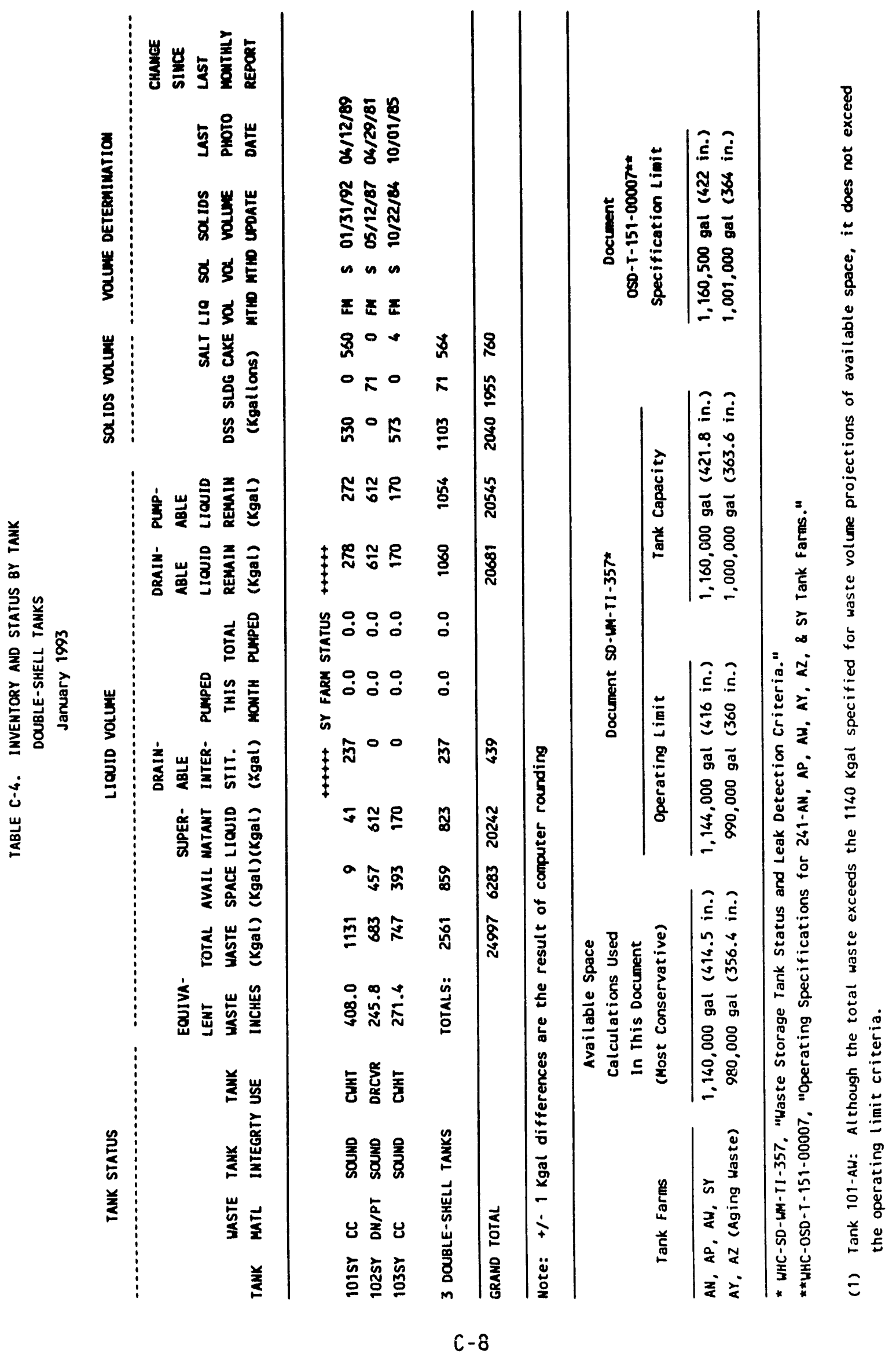




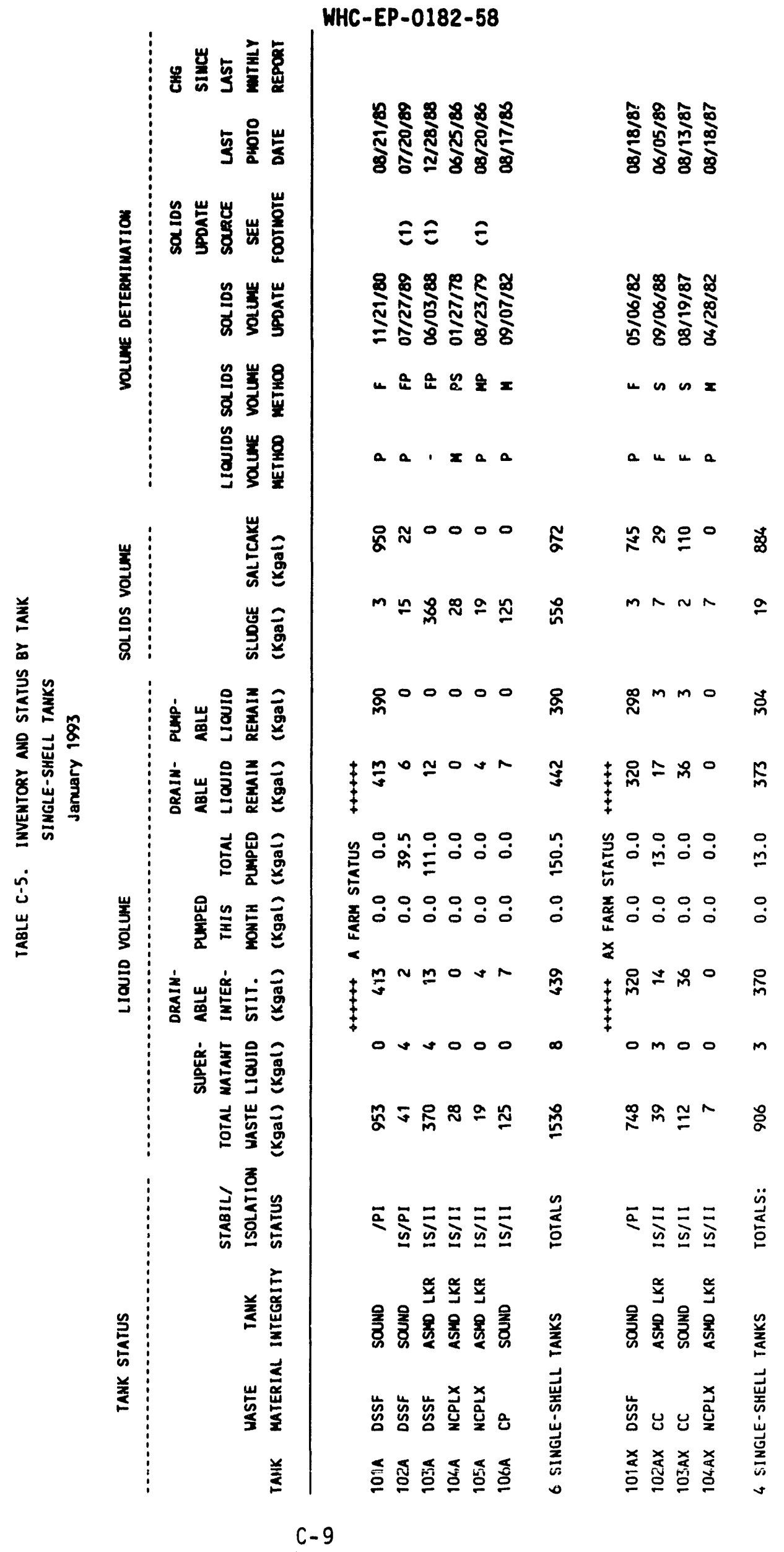




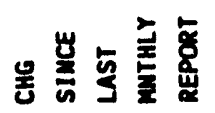

京旁宸

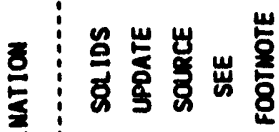

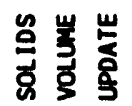

늘

名

总崖害

善高

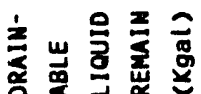

구은 은 赛

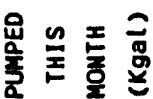

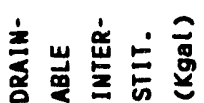

离衰言高

言容高

吾哭

差

뜬

美
WHC-EP-0182-58

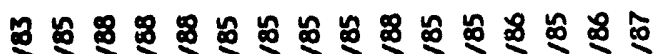

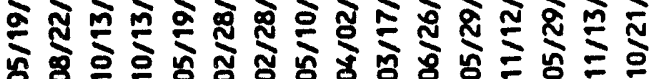

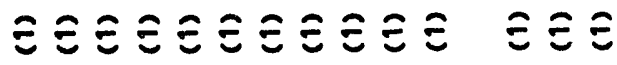

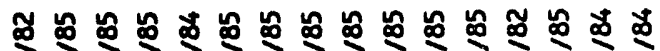

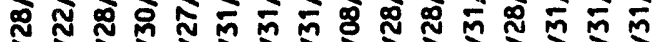
ชิ영 중
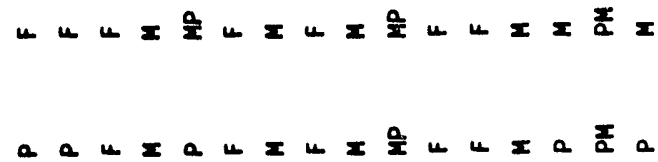

0.088000000000000

뜸ํำ

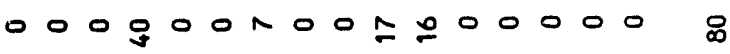

Horothnmannmamon

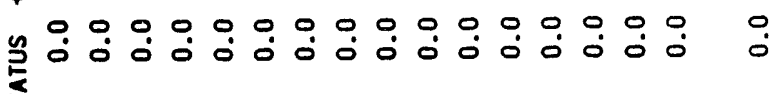

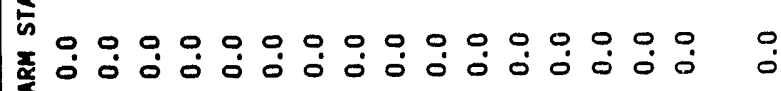
4

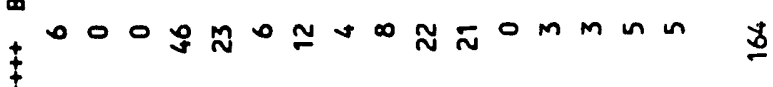
年

$000-0-00-m-0 \ldots$

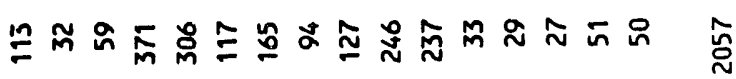

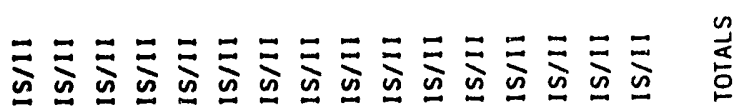

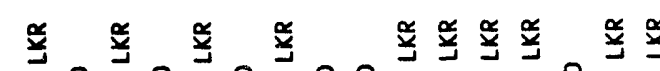

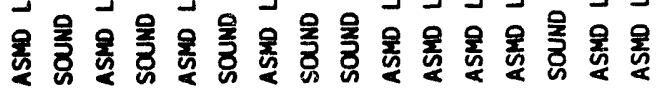

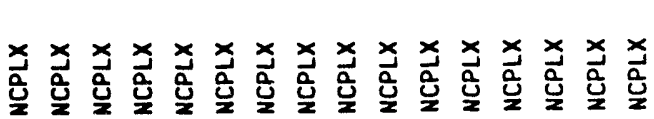

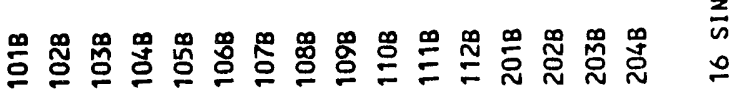
C -10 


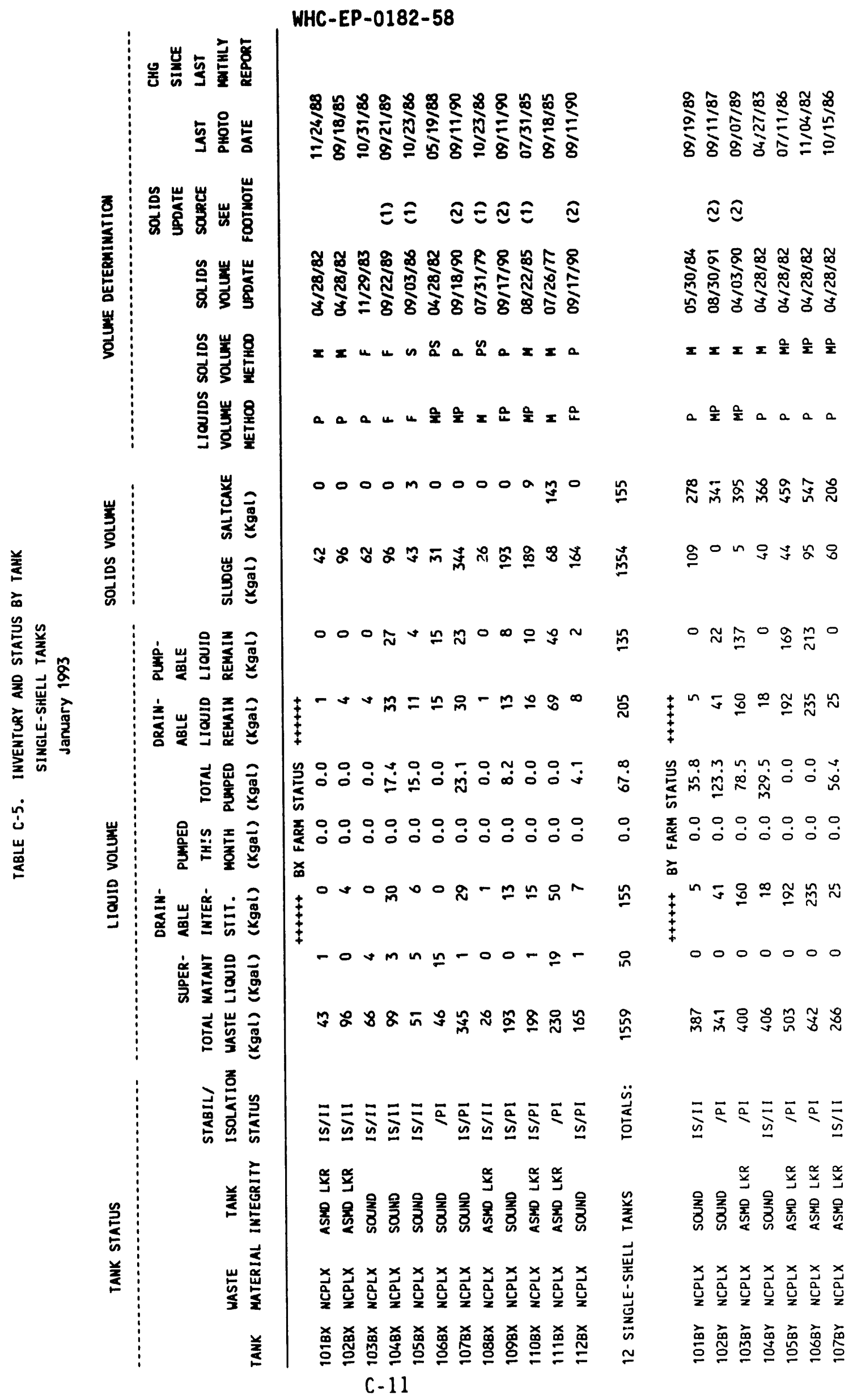




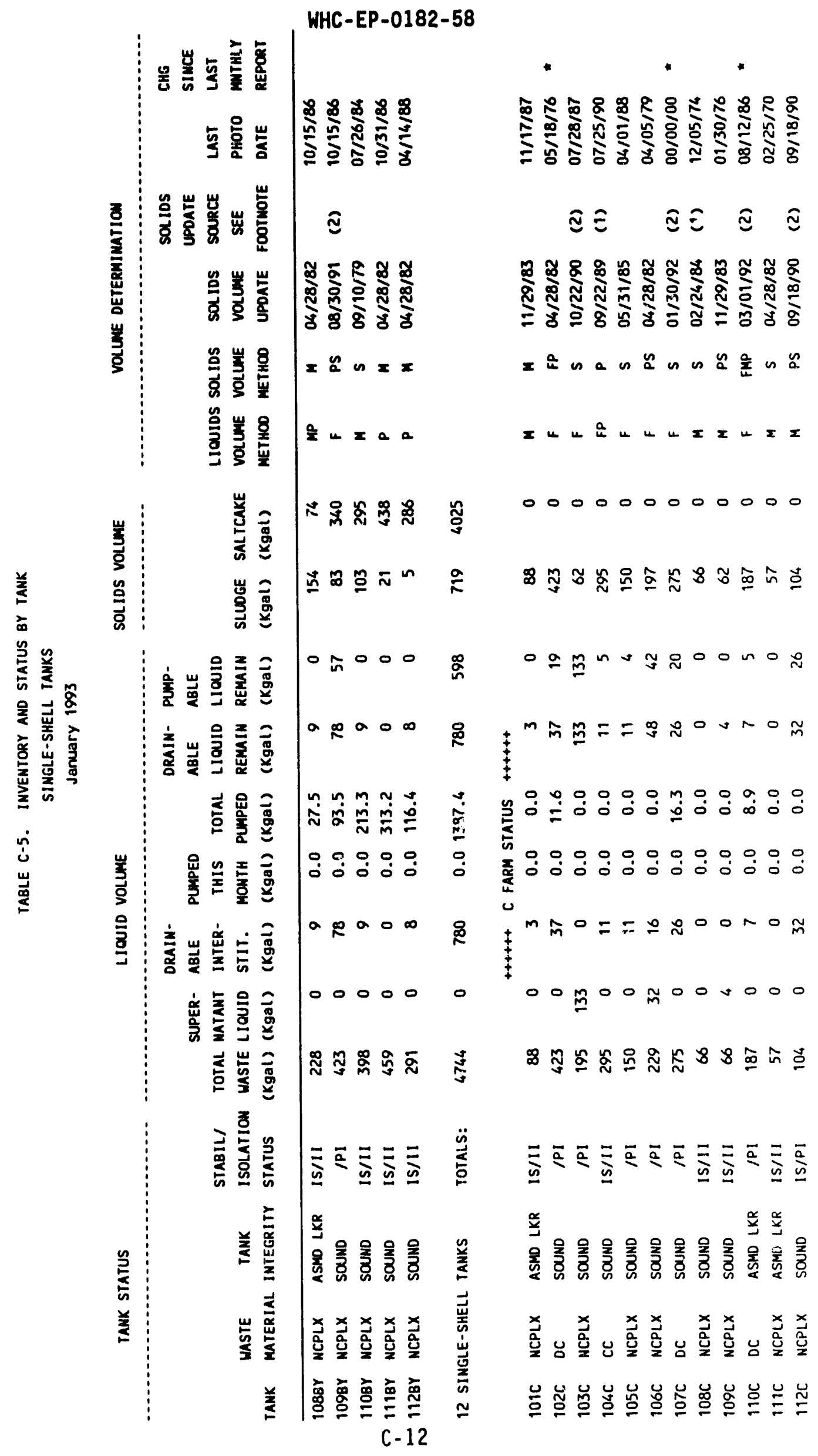




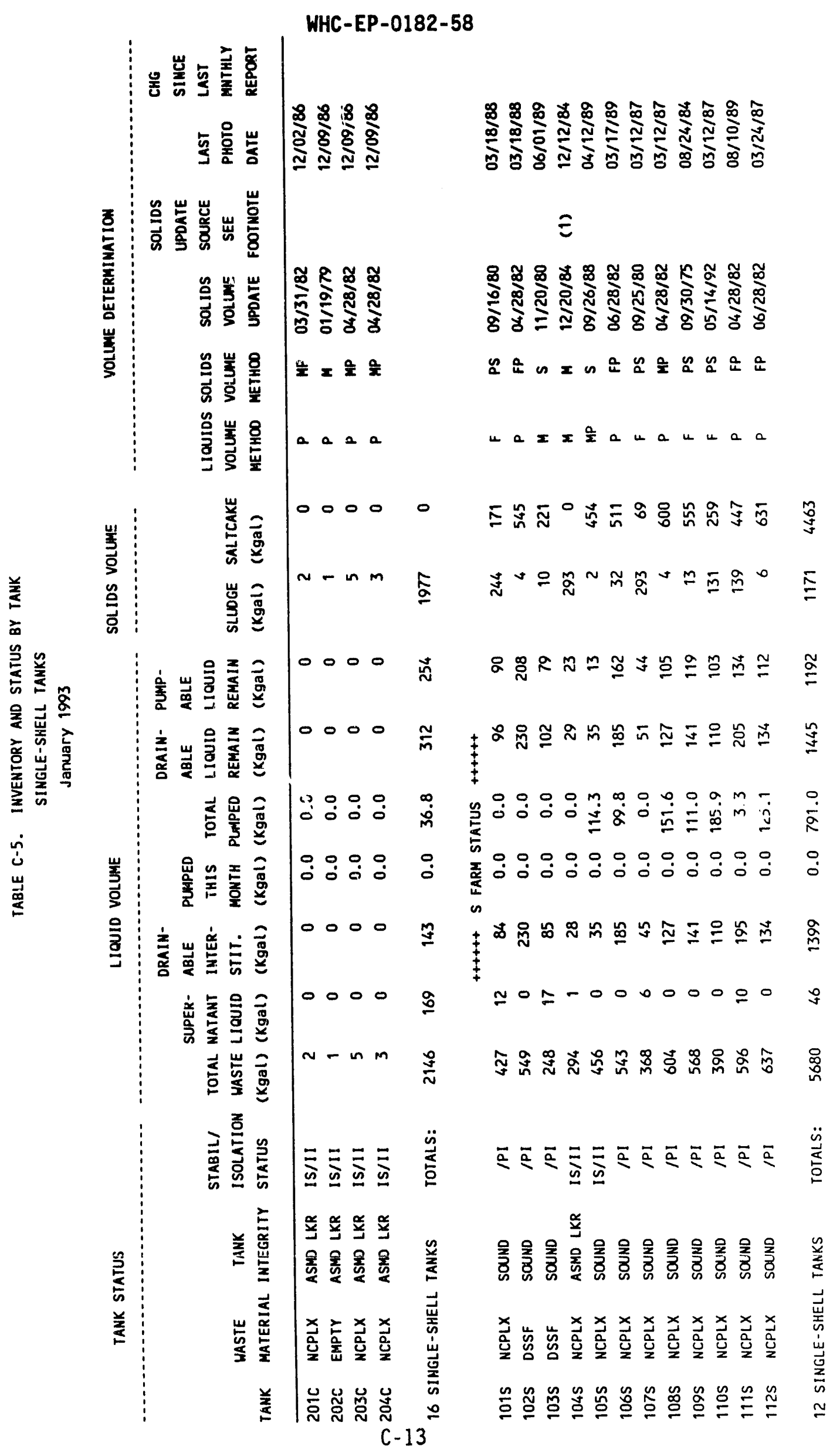




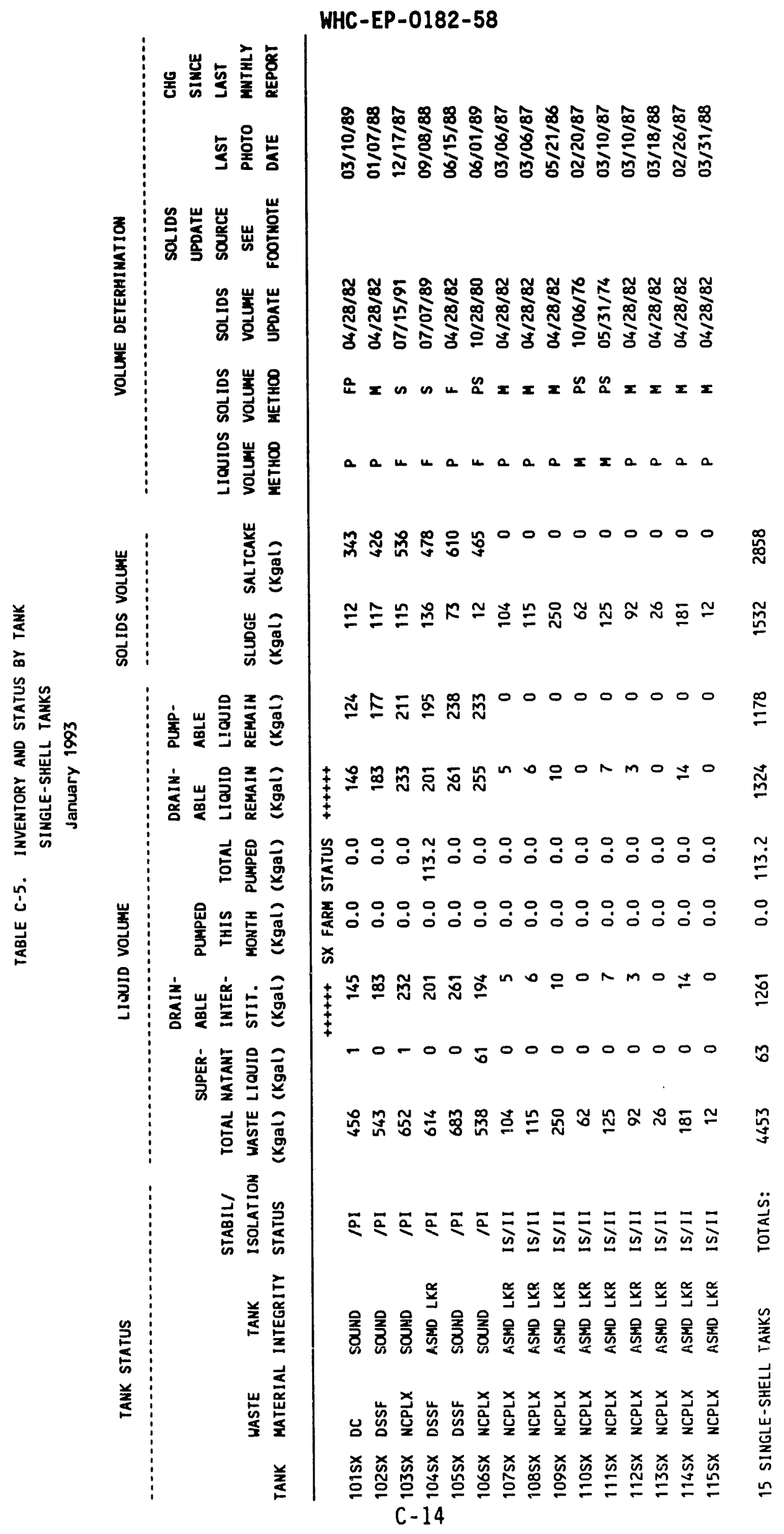


WHC-EP-0182-58

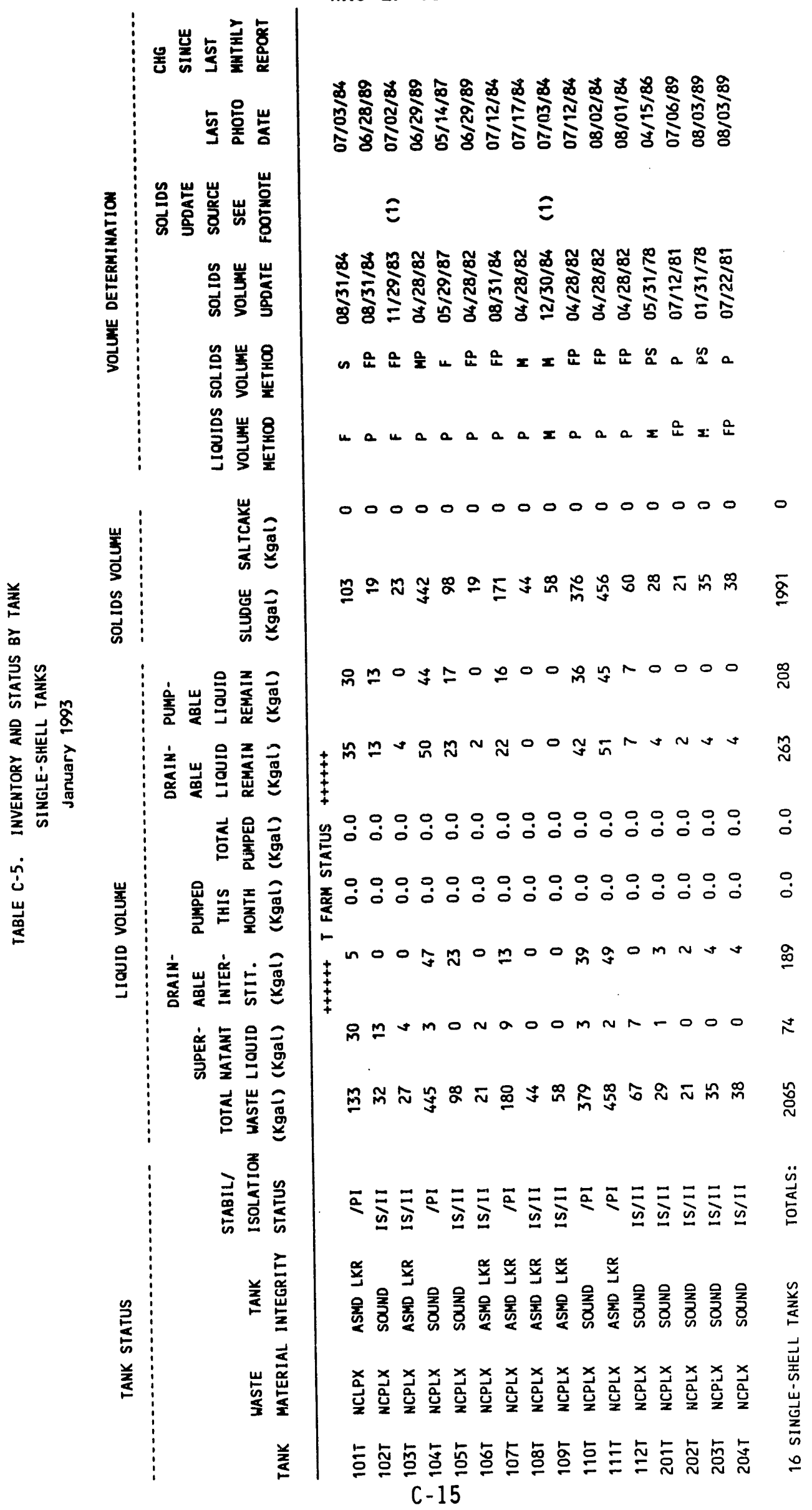




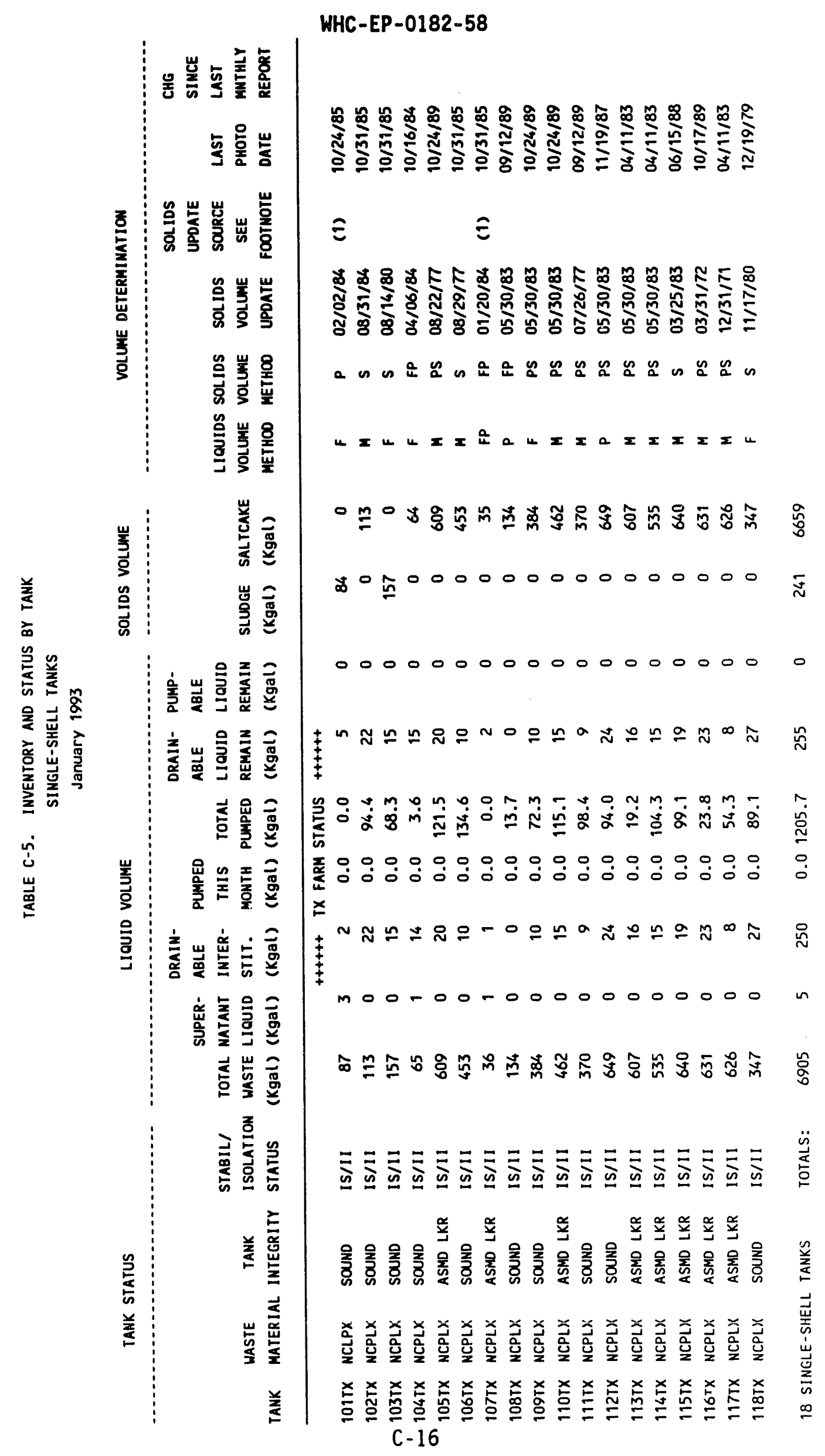




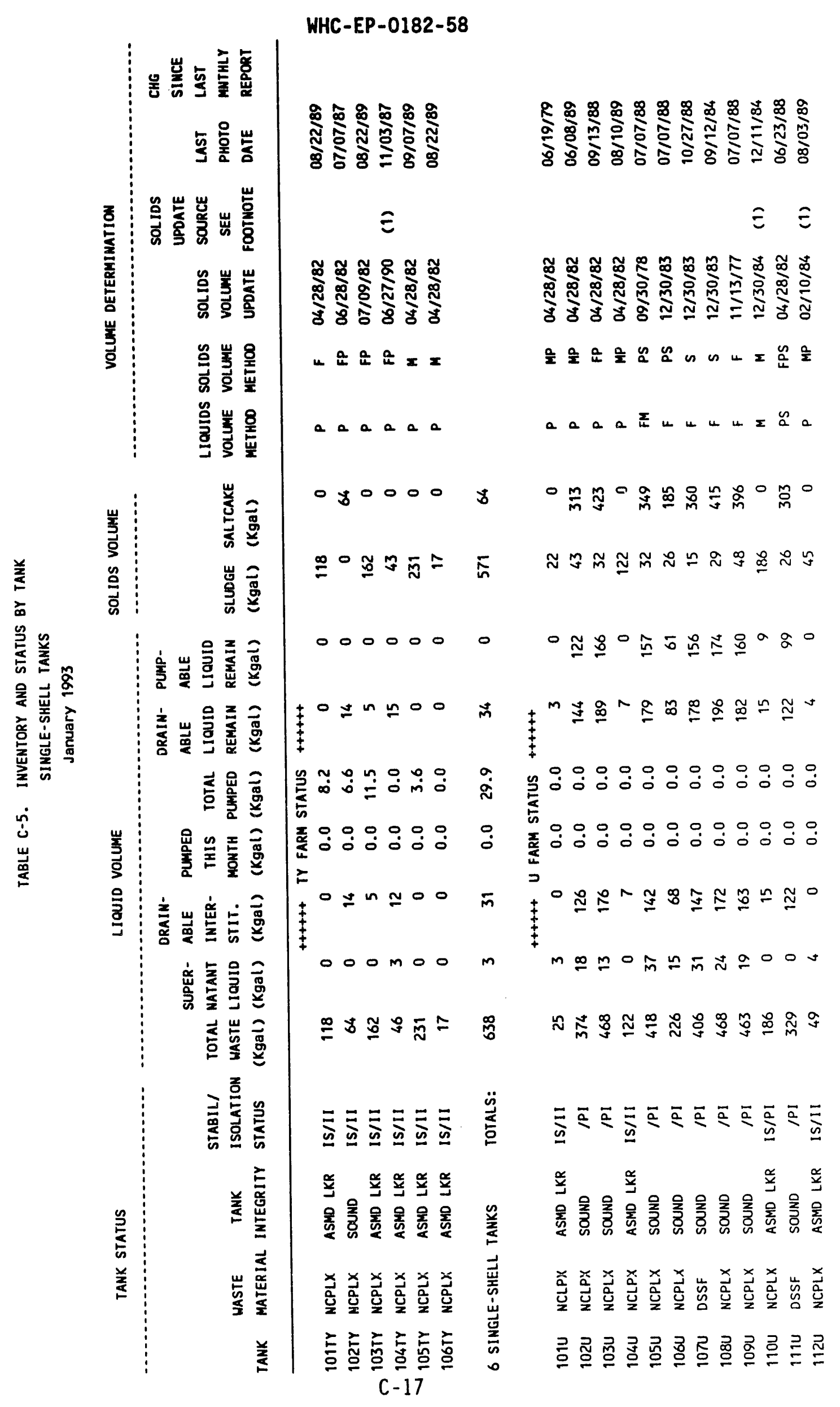




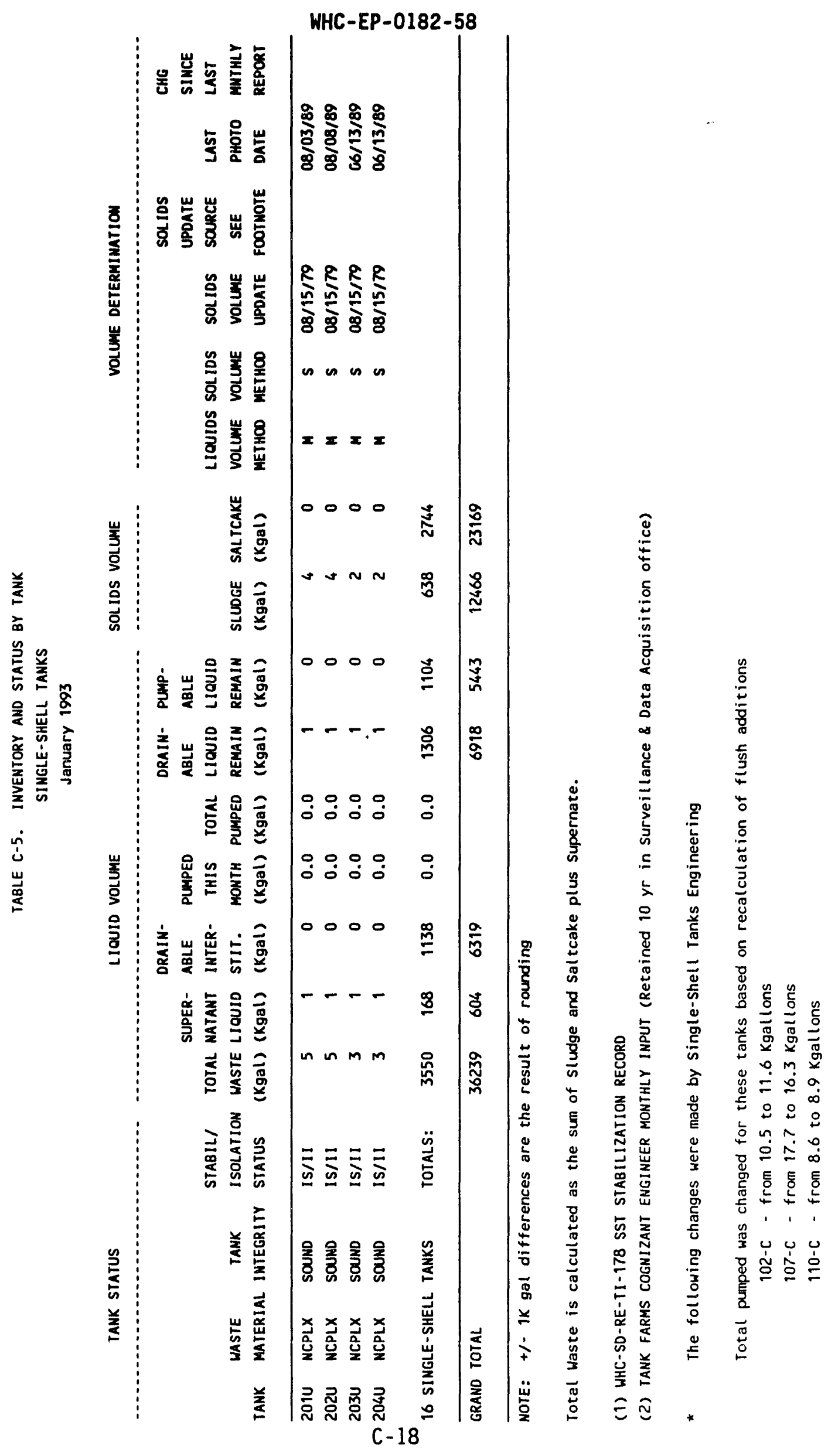


WHC-EP-0182-58

APPENDIX D

PERFORMANCE SUMMARY

D-1 
WHC-EP-0182-58

This page intentionally left blank. 


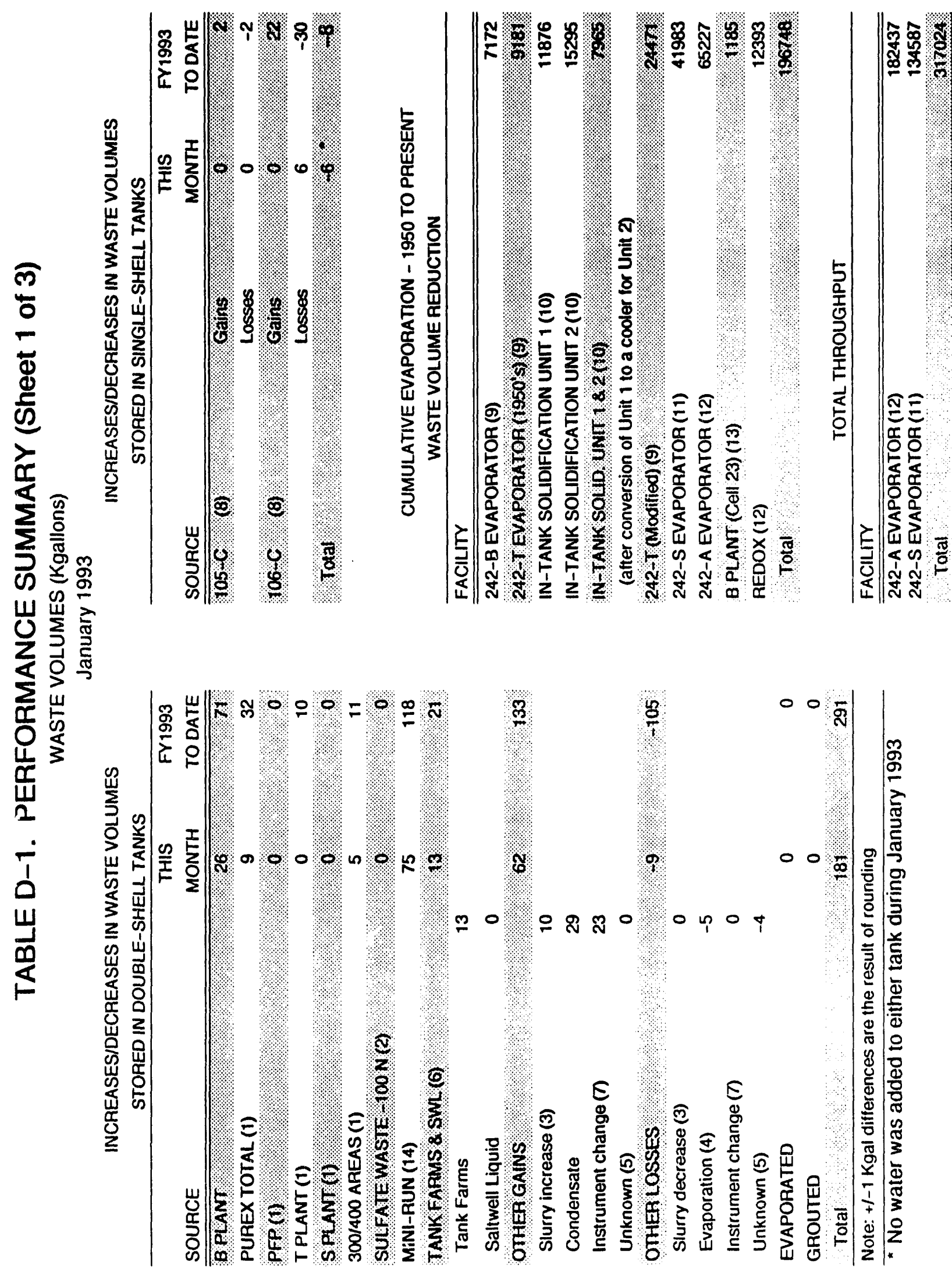

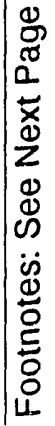


TABLE D-1. Performance Summary (Sheet 2 of 3 )

Footnotes:

\section{INCREASES/OECREASES IN WASTE VOLUMES}

(1) Including Flush

(2) Sulfate waste is generated from ion exchange backflushing and sand filter clean out, resulting in sulfate waste $\left(\mathrm{Na}_{2} \mathrm{SO}_{4}\right)$.

(3) Slurry increase/growth is caused by gas generation within the waste. The gas which is trapped in the waste expands in the tank causing the surface level and volume to increase. Slurry decrease results from the periodic release of gas in the waste.

(4) Aging waste tanks

(5) Unknown waste gains or losses may be the result of rounding calculations, clean water slowly leaking through a valve, changes in levels (expansion/constraction) because of to ambient temperature changes, different measuring devices being used by Tank Farm operators, transfers taking place during the end of the month, Tank Farm activities such as miscellaneous water additions not associated with facility waste generation, or the addition of water which is added to aging waste tanks and then evaporated off.

(6) Includes Tank Farms miscellaneous flushes (flushes are used to "clean out" pipelines and reduce personnel exposure, reduce potential for waste incompatibility, prevent line plugging, and reduce waste content of potential spills or leaks), and saltwell liquid, which results from pumping of single-shell tanks to double-shell tanks.

(7) Liquid level measurement instrument changes from the automatic FIC to manual tape (and vice versa) result in unusual gains or losses because the manual tape may rest on an uneven crust surface giving a different reading from that of the automatic FIC. These instrument changes are made when the automatic FIC is out of service and the reading from the manual tape is used for reporting purposes. The reported reading reverts back to the automatic FIC when it is repaired.

(8) Water is periodically added to $105-\mathrm{C}$ and $106-\mathrm{C}$ to provide evaporative cooling. Losses due to evaporation are calculated assuming a 11 losses are evaporative losses. Drywell monitoring for leak detection is done monthly on tank 105-C. Some drywells are monitored weekly and some are monitored every two weeks on tank 106-C. If there are any indications of a leak from these tanks, the assumption that all losses are due to evaporation will be reevaluated. 
TABLE D-1. Performance Summary (Sheet 3 of 3 )

\section{WASTE VOLUME REDUCTION}

(9) Currently inoperative. These evaporator systems (242-B and 242-T) were installed in 1952 in each of the two operating areas to remove water from the waste, and ran for approximately $4 \mathrm{yr}$ after which both units were shut down. The 242-T Evaporator was reactivated in December 1965, and shut down again in Aprit 1976.

(10) Currently inoperative. These two in-tank solidification (ITS) units provided in-tank heating to promote in-tank boiling or evaporation. The ITS Unit 1 started up March 1965, and ITS Unit 2 started up February 1968. In August 1971, ITS Unit 1 was converted from an evaporator to a cooler for ITS Unit 2. Both units were shut down June 1974.

(11) Currently inoperative. The 242-S Evaporator-Crystallizer was started up November 1973, and shut down March 1980, when its processing campaign was completed. It is in standby mode with no future mission. This evaporator operates under a vacuum, employing evaporative concentration with subsequent crystallization and precipitation of salt crystals.

(12) Currently inoperative. The 242-A Evaporator-Crystallizer was started up March 1977, and shut down April 1989 because of regulatory issues, and has remained shut down for subsequent upgrading. The restart schedule specifies January 4, 1993, as the projected start-up date. This evaporator operates under a vacuum, employing evaporative concentration with subsequent crystallization and precipitation of salt crystals (forming saltcake).

(13) Currently inoperative. Additional concentration of wastes was completed by using the concentrators at REDOX and B Plant. The REDOX concentrator was used from July 1967 to June 1972, while the B Plant concentrator was used from July 1967 to February 1968.

(14) Waste generated for training and testing purposes prior to Evaporator restart 
WHC-EP-0182-58

This page intentionally left blank

D.6 
WHC-EP-018\%-58

\section{APPENDIX E}

\section{LIQUID STATUS AND PUMPABLE LIQUID}

REMAINING IN TANKS 
WHC-EP-0182-58

This page intentionally left blank. 
WHC-EP-0182-58

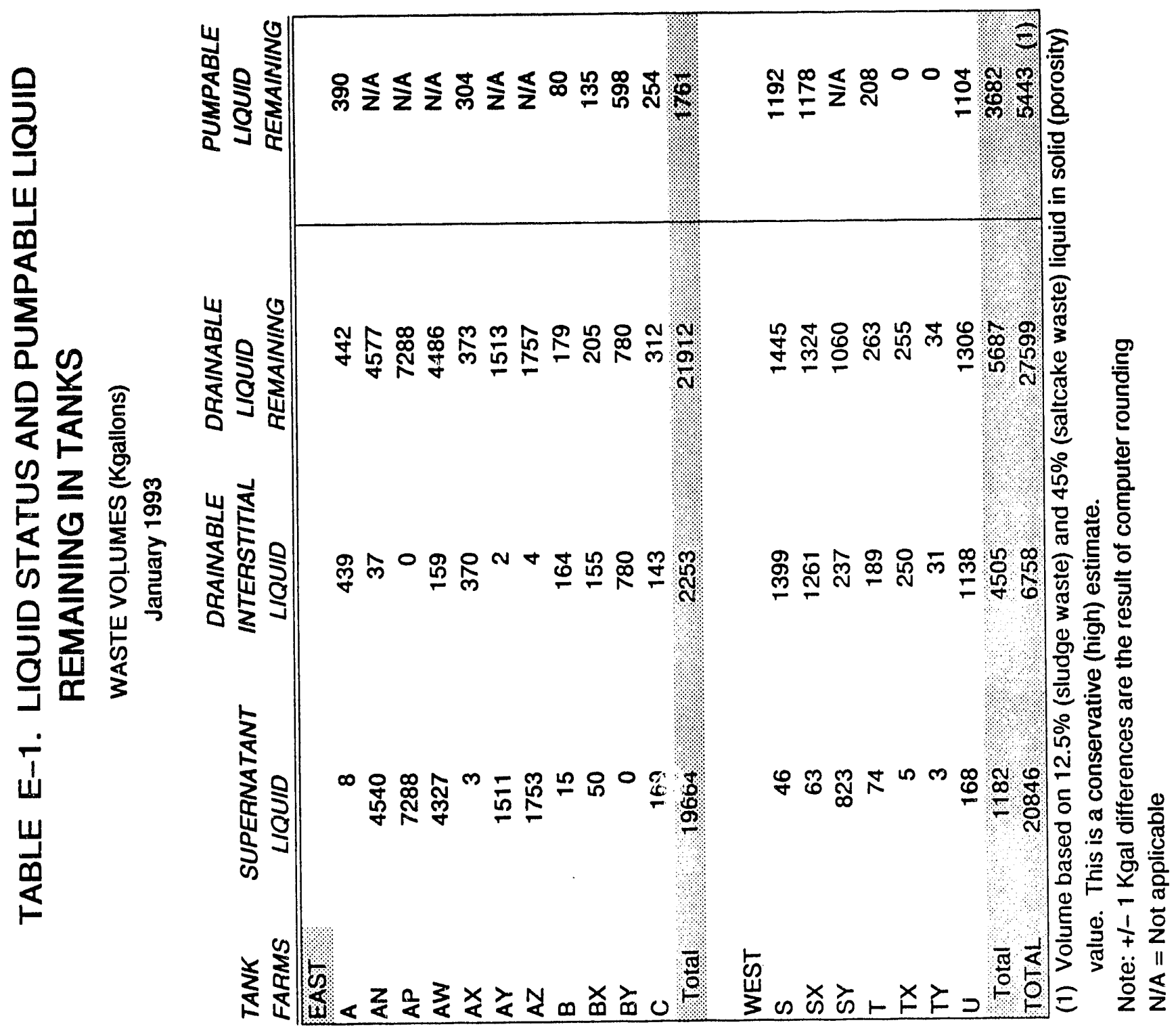


WHC-EP-0182-58

This page intentionally left blank.

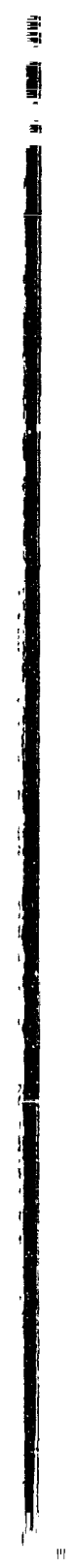


WHC-EP-0182-58

\section{APPENDIX F}

PUMPING RECORD 
WHC-EP-0182-58

This page intentionally left blank.

$F-2$ 


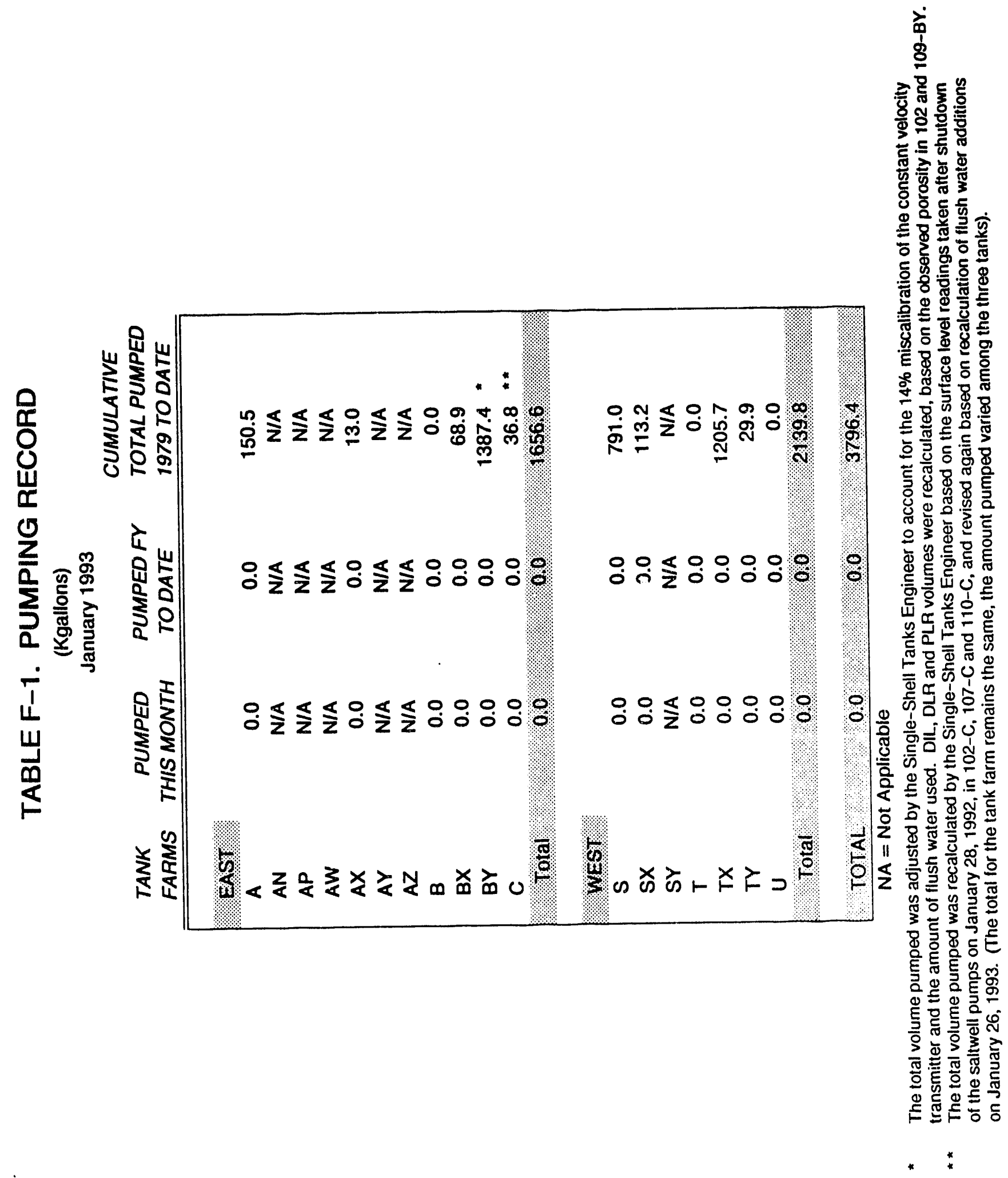


WHC-EP-0182-58

This page intentionally left blank. 
WHC-EP-0182-58

APPENDIX G

CATCH TANKS AND SPECIAL

SURVEILLANCE FACILITIES 
WHC-EP-0182-58

This page intentionally left blank.

G-2 

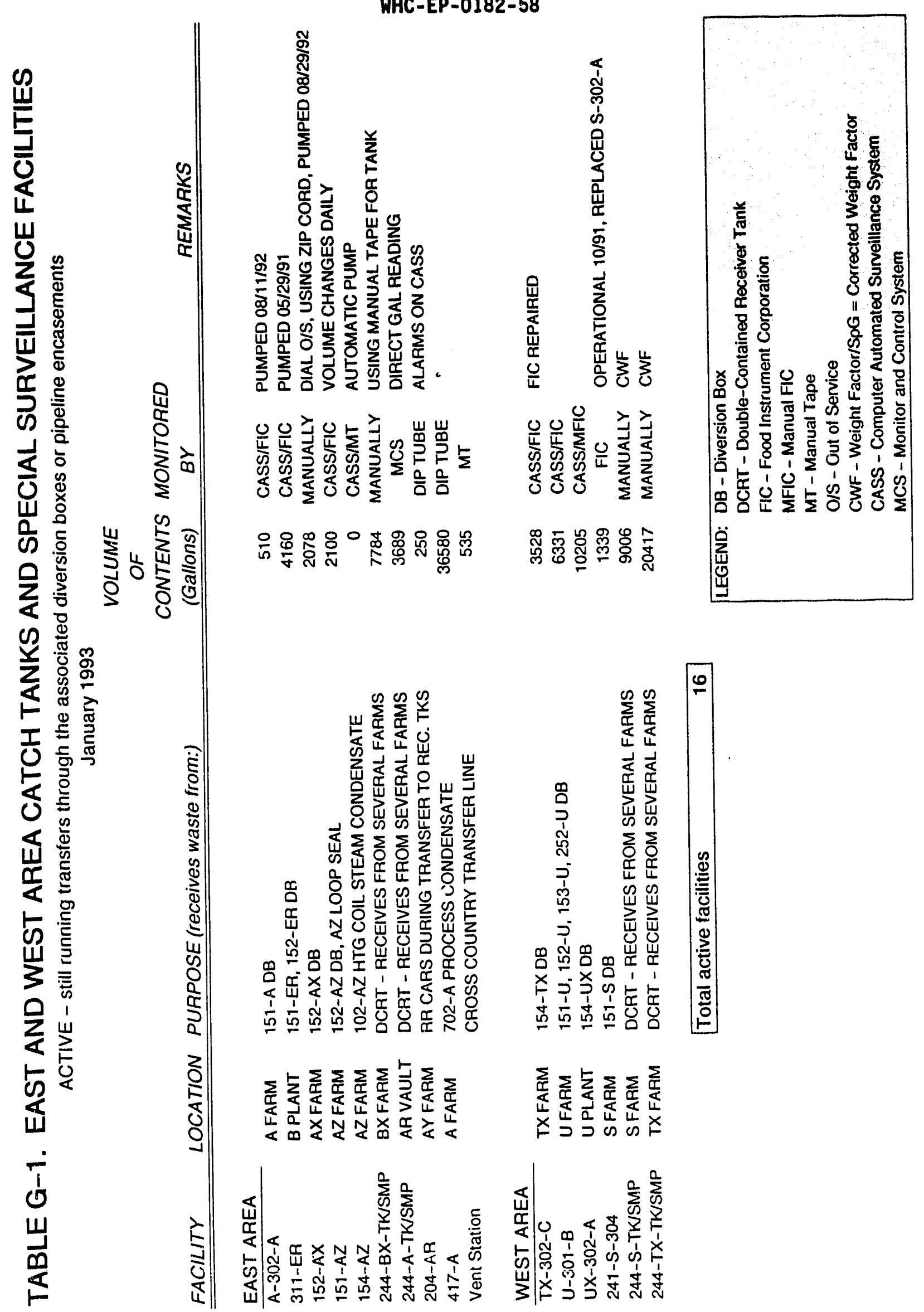
WHC-EP-0182-58

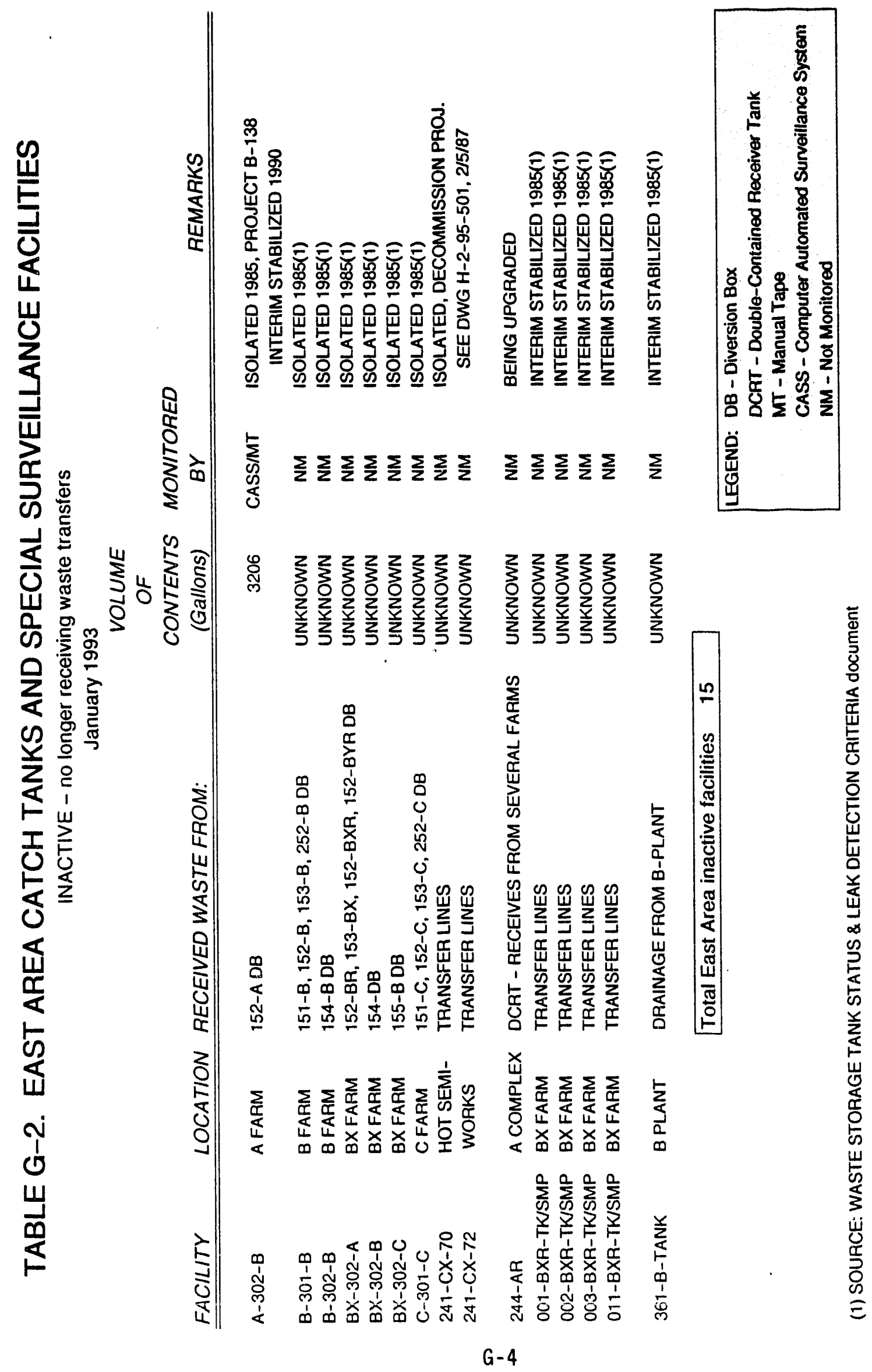




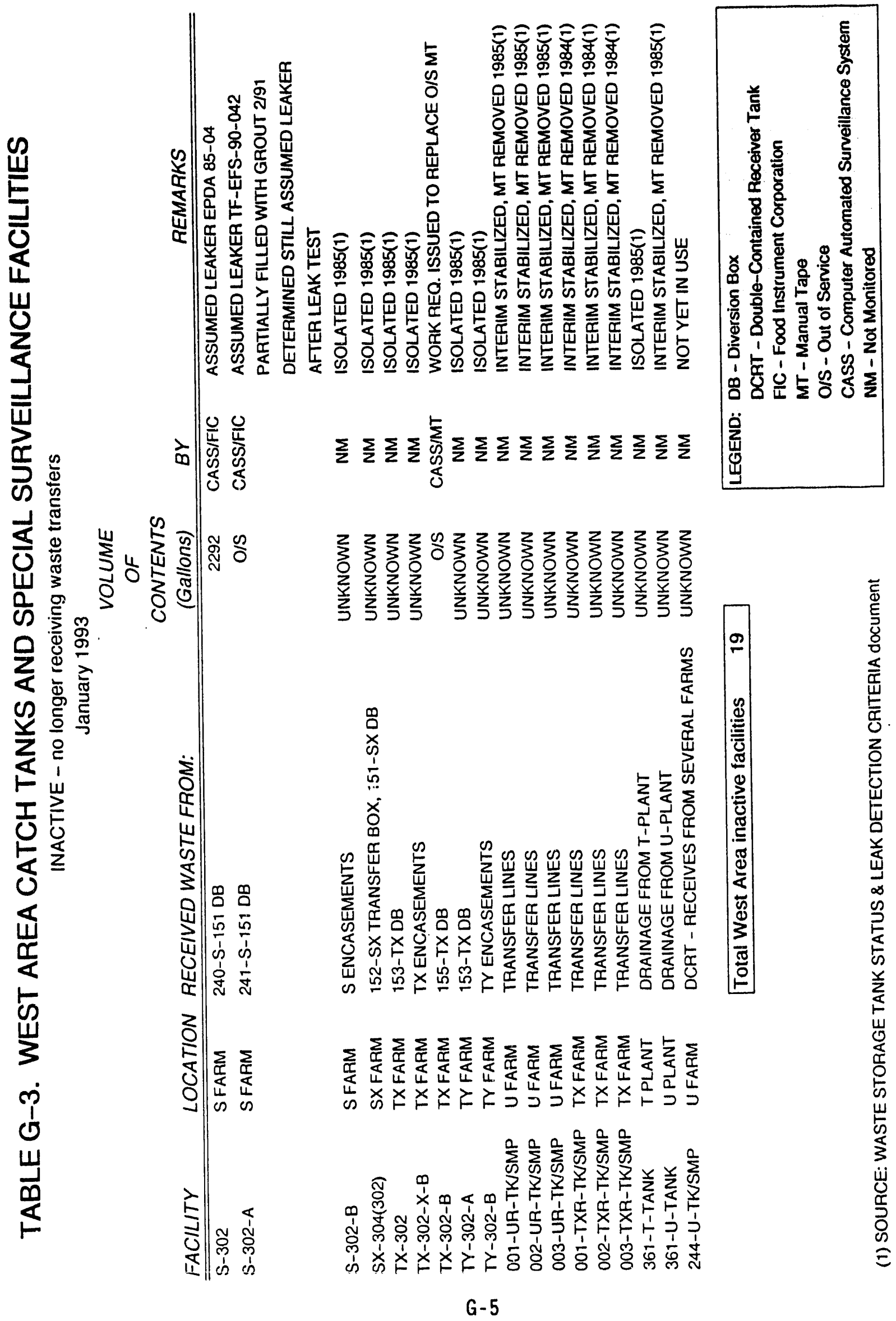


WHC-EP-0182-58

This page intentionally left blank. 
WHC-EP-0182-58

\section{APPENDIX H}

\section{LEAK VOLUME ESTIMATES}


WHC-EP-0182-58

This page intentionally left blank. 
WHC-EP-0182-58

TABLE H-1. SINGLE-SHELL TANK LEAK VOLUME ESTIMATES (4) (Sheet 1 of 5)

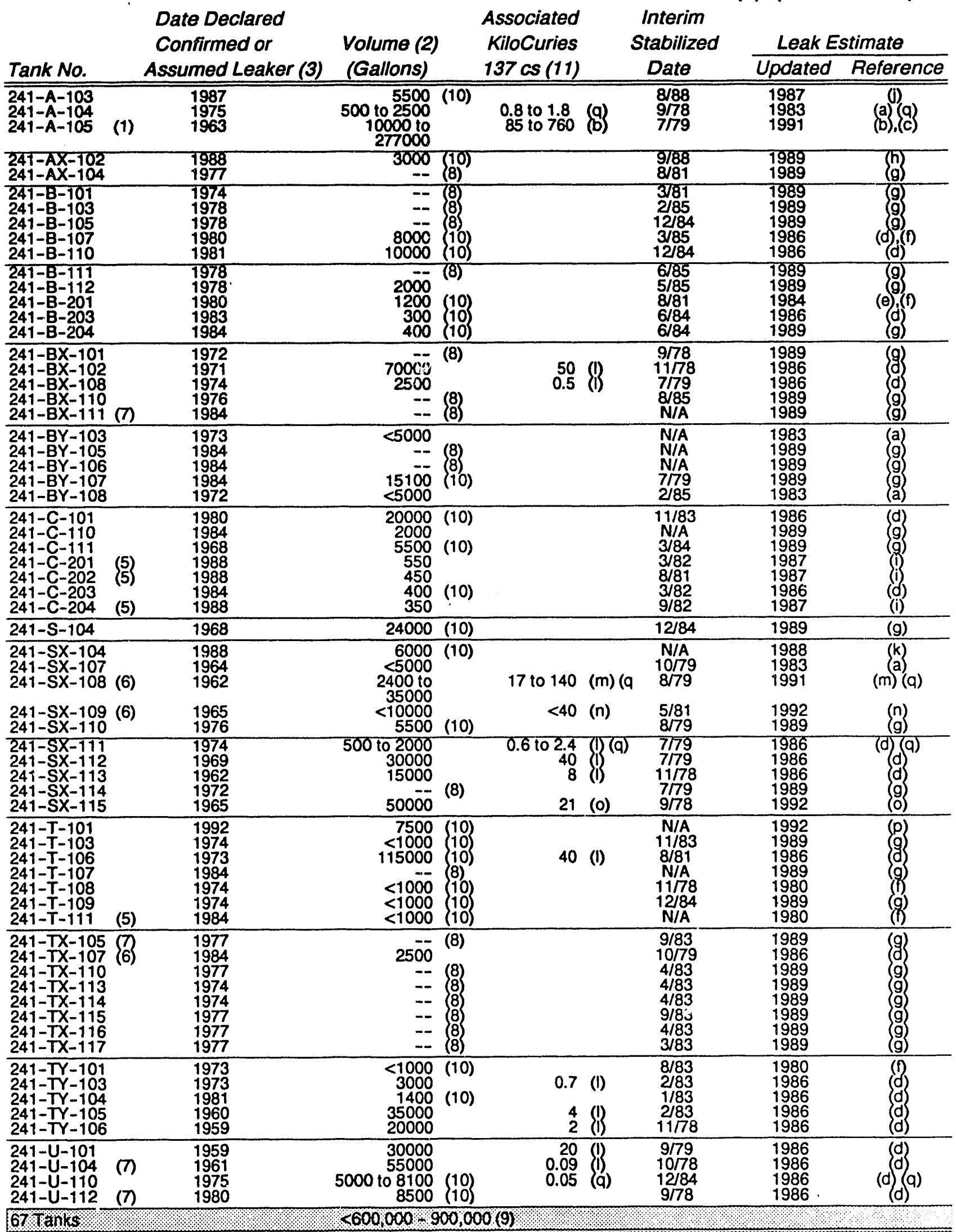

N/A = not applicable (not yet interim stabilized)

FOOTNOTES: SEE NEXT PAGE 
TABLE H-1. Single-She11 Tank Leak Volume Estimates (Sheet 2 of 5 ).

\section{Footnotes:}

(1) Current estimates (see reference b) are that $610 \mathrm{Kgal}$ of cooling water was added to Tank 241-A-105 from November 1970 to December 1978 to aid in evaporative cooling. In accordance with Dangerous haste Regulations (Washington Administrative Code 173-303-070 (2)(a)(ii), as amended, Washington State Department of Ecology, 1990, 01ympia, Washington), any of this cooling water that has been added and subsequently leaked from the tank must be classified as a waste and should be included in the total leak volume. In August 1991, the leak volume estimate for this tank was updated and moved into compliance with the WAC regulations. Previous estimates excluded the cooling water leaks from the total leak volume estimates because the waste content (concentration) in the cooling water which leaked should be much less than the original liquid waste in the tank (the sludge is relatively insoluble). The total leak volume estimate in this report (10 Kgal to $277 \mathrm{Kgal})$ is based on the following (see References).

1. Reference (b) contains an estimate of $5 \mathrm{Kgal}$ to $15 \mathrm{Kgal}$ for the initial leak prior to August 1968.

2. Reference (b) contains an estimate of $5 \mathrm{Kgal}$ to $30 \mathrm{Kgal}$ for the leak while the tank was being sluiced from August 1968 to November 1970.

3. Reference (b) contains an estimate of $610 \mathrm{Kgal}$ of cooling water added to the tank from November 1970 to December 1978 but it was estimated that the leakage was small during this period. This reference contains the statement "Sufficient heat was generated in the tank to evaporate most, and perhaps nearly all, of this water." This results in a low estimate of zero gallons leakage from November 1970 to December 1978.

4. Reference (c) contains an estimate that 378 to $410 \mathrm{Kgal}$ evaporated out of the tank from November 1970 to December 1978. Subtracting the minimum evaporation estimate from the cooling water added estimate provides a range from 0 to $232 \mathrm{Kgal}$ of cooling water leakage from November 1970 to December 1978.

\section{Low Estimate High Estimate}

$\begin{array}{lrr}\text { Prior to August } 1968 & 5,000 & 15,000 \\ \text { August } 1968 \text { to November 1970 } & 5,000 & 30,000 \\ \text { November } 1970 \text { to December } 1978 & \frac{0}{27,000} & \frac{232,000}{277,000} \\ \text { Totals } & 10,000 & \end{array}$

(2) These leak volume estimates do not include (with some exceptions), such things as: (a) cooling/raw water leaks, (b) intrusions (rain infiltration) and subsequent leaks, (c) leaks inside the tank farm but not through the tank liner (surface leaks, pipeline leaks, leaks at the joint for the overflow or fill lines, etc.), and (d) leaks from catch tanks, diversion boxes, encasements, etc. 
TABLE H-1. Single-She11 Tank Leak Volume Estimates

(Sheet 3 of 5 )

(3) In many cases, a leak was suspected long before it was identified or confirmed. In 1984, the criteria designations of "suspected leaker," "questionable integrity," "confirmed leaker," "declared leaker," "borderline," and "dormant," were merged into one category now reported as "assumed leaker." See reference ( $f$ ) for explanation of when, how long, and how fast some of the canks leaked.

(4) There is an effort currently in progress to reevaluate these leak volume estimates. During the FY 1993 funding reviews, this reevaluation of leak volumes was given a priority which resulted in this activity no longer being funded. The priority versus funding will be reevaluated prior to FY 1994.

(5) The leak volume estimate date for these tanks is before the "declared leaker" date because the tank was in a "suspected leaker" or "questionable integrity" status; however, a leak volume had been estimated prior to the tank being reclassified.

(6) The increasing radiation levels in drywells and laterals associated with these three tanks could be indicative of a continuing leak or movement of existing radionuclides in the soil. There is no conclusive way to confirm these observations.

(7) These four tanks also show slight indications of continuing leaks or movement of radionuclides in the soil.

(8) Methods were used to estimate the leak volumes from these 19 tanks based on the assumption that their cumulative leakage is approximately the same as for 18 of the 24 tanks identified in footnote (10). For more details see reference $(\mathrm{g})$. The total leak volume estimate for these tanks is $150 \mathrm{Kgal}$ (rounded to the nearest $10 \mathrm{Kgal}$ ), for an average of approximately $8 \mathrm{Kgal}$ for each of the 19 tanks.

(9) The total has been rounded to the nearest $50 \mathrm{Kgal}$. Upperbound values were used in many cases in developing these estimates. It is likely that some of these tanks have not actually leaked.

(10) Leak volume estimate is based solely on observed liquid level decreases in these tanks. This is considered to be the most accurate method for estimating leak volumes.

(11) The curie content listed is as listed in the reference document and is not decayed to a consistent date; therefore, a cumulative total is inappropriate. 
TABLE H-1. Single-Shell Tank Leak Volume Estimates.

(Sheet 4 of 5 )

References:

(a) Murthy, K.S., et al, June 1983, Assessment of Single-Shell Tank Residual Liquid Issues at Hanford Site, Washington, PNL-4688, Pacific Northivest Laboratory, Richland, Washington.

(b) WHC, 1991a, Tank 241-A-105 Leak Assessment, WHC-MR-0264, Westingholise Hanford Company, Richland, Washington.

(c) WHC, 1991b, Tank 241-A-105 Evaporation Estimate 1970 Through 1978, WHC-EP-0410, Westinghouse Hanford Company, Richland, Washington.

(d) Smith, D. A., January 1986, Single-Shell Tank I solation Safety Analysis Report, SD-WM-SAR-006, Rev. 1, Westinghouse Hanford Company, Richland, Washington.

(e) McCann, D. C., and T. S. Vail, September 1984, Waste Status Summary, RHO-RE-SR-14, Rockwell Hanford Operations, Richland, Washington.

(f) Catlin, R. J., March 1980, Assessment of the Surveillance Program of the High-Level Waste Storage Tanks at Hanford, Hanford Engineering Development Laboratory, Richland, Washington.

(g) Baumhardt, R. J., May 15, 1989, Letter to R. E. Gerton, U.S. Department of Energy-Richland Operations Office, Single-Shell Tank Leak Volumes, Westinghouse Hanford Company, Richland, Washington.

(h) WHC, 1990a, Occurrence Report "Surface Level Measurement Decrease in Single-Shell Tank 241-AX-102," WHC-U0-89-023-TF-05, Westinghouse Hanford Company, Richland, Washington.

(i) Groth, D. R., July 1, 1987, Internal Memorandum to R. J. Baumhardt, Liquid Level Losses in Tanks 241-C-201, -202 and-204, 65950-87-517, Westinghouse Hanford Company, Richland, Washington.

(j) Groth, D. R. and G. C. Owens, May 15, 1987, Internal Memorandum to J. H. Roecker, Tank 103-A Integrity Evaluation, Westinghouse Hanford Company, Richiand, Washington.

(k) Campbe11, G. D., July 8, 1988, Internal Memorandum to R. K. Welty, Engineering Investigation: Interstitial Liquid Level Decrease in Tank 241-SX-104, 13331-88-416, Westinghouse Hanford Company, Richland, Washington.

(1) ERDA, 1975, Final Environmental Statement Waste Management Operations, Hanford Reservation, Richland, Washington, ERDA-1538, 2 vols., U.S. Energy Research and Development Administration, Washington, D.C.

(m) WHC, 1992a, Tank 241-SX-108 Leak Assessment, WHC-MR-0300, Westinghouse Hanford Company, Richland, Washington.

(n) WHC, 1992b, Tank 241-SX-109 Leak Assessment, WHC-MR-0301, Westinghouse Hanford Company, Richland, Washington. 


\section{WHC-EP-0182-58}

TABLE H-1. Single-Shell Tank Leak Volume Estimates.

(Sheet 5 of 5 )

(0) WHC, 1992c, Tank 241-SX-115 Leak Assessinent, WHC-MR-0302, Westinghouse Hanford Company, Richland, Washington.

(p) WHC, 1992d, Occurrence Report, "Apparent Decrease in Liquid Leve1 in Single She11 Underground Storage Tank 241-T-101, Leak Suspected; Investigation Continuing," RL-WHC-TANKFARM-1992-0073, Westinghouse Hanford Company, Richland, Washington.

(q) WHC-1990b, A History of the 200 Area Tank Farms, WHC-MR-0132, Westinghouse Hanford Company, Richland, Washington. 
WHC-EP-0182-58

This paye intentionally left blank.

H-8 


\section{DISTRIBUTION}

\section{Number of copies}

OFFSITE - USA

Congress of the United States

House of Representatives

1111 Longworth Building

Washington, DC 20515-3703

Ron Wyden, Member of Congress, 3rd District Josh Kardon, Legislative Director

20

U. S. Department of Energy-Headquarters

1000 Independence Avenue, SW

Washington, D. C. 20585

Emile Bernard

H. Calley

H. Eckert

Teresa Fryberger

Sherry Gibson

A. Griffith

L. Gunn

D. Gupta

J. S. Kang

Kenneth Lang

J. C. Lehr

G. Mellinger

John Morrey

C. O'De11

C. Pepson

J. C. Tseng

S. Woodbury

19901 Germantown Rd,

Germantown, MD 20585
R. Lasky
J. Psaras
P. Worthington

$E M-50$

$E M-36$

EM-36

$E M-542$

$E M-55$

$E M-322$

EM-36

EM-36

EM-351

$E M-36$

$E M-442$

EM-36

(Pac. NW Labs)

EM-36

$E M-36$

EM-36

EH-222

TREV II

TREV I I

TREV II

TREV II

TREV II

TREV II $/ 343$

TREV II

TREV II

TREV II $/ 368$

TREV II

TREV II $/ 160$

TREV II

TREV II

TREV II

TREV II

TREV II $/ 366$

FORS/3G-092

EH-32.1 GTN/G-115

NS-20 GTN

NS-20 GTN

U. S. Department of Energy - Oak Ridge Operations Office P. 0. Box 2001

Oak Ridge, TN 37831

W. D. Adams EW-40 
WHC-EP-0182-58

\section{Distribution - continued}

U. S. Department of Energy - Savannah River Site

P. 0. Box A

Aiken, SC 29808

C. Anderson

$707-\mathrm{H}$

Michael Chandler

$703-\mathrm{H}$

Mazen Shurrab

T. C. Temple

$704-\mathrm{H}$

L. Sjostrom

W. R. West

$V$. Wheeler

$704-8 \mathrm{H}$

704-S

1

U. S. Department of Energy - Idaho Operations

785 D. 0. E. Place

Idaho Falls, ID 83402

W. Sato

MS - $11-18$

1

U. S. Environmental Protection Agency Region 10

712 Swift Boulevard, Suite 5

Richland, WA 99352

P. T. Day

3

Washington State Department of Ecology

Nuclear \& Mixed Waste Management

719 Sleater-Kinney Road S. E., Suite 200

Lacey, WA 98503-1138

R. Stanley

M. T. Gordon

7601 W. Clearwater \#102

Kennewick, WA 99336

Greta Davis

1

Washington State Department of Health

Radiation Protection Section

Industrial Park Building 5, LE-13

01ympia, WA 98504

A. Conklin

1

Oregon State Department of Energy

625 Marion St. N.E.

Salem, OR 97310

Janet Franco 
WHC-EP-0182-58

\section{Distribution - continued}

Oregon State Water Resources Department Ground Water Hanford Studies 3850 Portland Road Salem, OR 97310

R. 0. Patt

Lawrence Livermore National Laboratory Box 808, East Avenue Livermore, CA 94550

B. C. Hudson $L-221$

Oak Ridge National Laboratory P. 0. Box 2009

Oak Ridge, TN 37831-6385

C. Forsberg

MS -6495

T. S. Kress

MS -8088

B1dg 9108

Chemical Technology Division P. 0. Box 2008

Emory D. Collins

6

Los Alamos National Laboratory P. 0. Box 1663

Los Alamos, NM 87545

Stephen Agnew

Group INC-14

Phyll is Baca

T. Larson

Sylvia Lee

A. Nuels

$\mathrm{K}-557$

H. Sullivan

$\mathrm{N}-6$

$N-6$

5

Brookhaven National Laboratory Upton, NY 11973

K. K. Bandyopadhyay, B1dg 475-C

M. K. Kaisser, B1dg 475-C

P. D. Kalb, Bidg. 703

M. Reich, Bldg 475-C

J. R. Weeks, Bidg 197-C

1 Brookhaven National Laboratory 1409 Jan Drive Wilmington, DE 19803

Michael Streicher 
WHC-EP-0182-58

Distribution - continued

Sandia National Laboratories

1515 Eubank, NE

P. 0. Box 5800

A1buquerque, NM 87185

Scott Slezak, Division 6402

Leon D. Chapman, Program Manager

Industrial Waste Reduction Program

1

Massachusetts Institute of Technology

77 Massachusetts Avenue

Cambridge, MA 02139

Mujid S. Kazimi

Professor and Head

Department of Nuclear Engineering

2

BDM International, Inc.

20030 Century B1vd, Suite 101

Germantown, MD 20874

P. Kiang

K. J. Mahoney

3

SAIC

20300 Century Blvd.

Germantown, MD 20874

J. Bunting

J. R. Pearring

R. A. Wullaert

3

SAIC

20300 Century Blvd.

Germantown, MD 20874

J. Bunting

J. R. Pearring

R. A. Wullaert

1

102 Windham Road

Oak Ridge, TN 37830

D. 0. Campbe11

1

SAIC

1845 Terminal Drive, Suite 130

Richland, WA 99352

J. Mishima 
WHC-EP-0182-58

Distribution - continued

Harvard School of Public Health

665 Huntington Avenue

Boston, MA 02115

M. First

1

Confederated Tribes, Umatilla Indian Reservation

P. 0. Box 638

Pendleton, OR 97801

Rick George

5

West Valley Nuclear Services Co.

P. 0. Box 191

West Valley, NY 14171

K. K. Gupta

MS -49

S. Ketola

MS -191

D. K. Ploetz

MS -305

Ram Shukla

Don Stroud

1

General Accounting Office

P. 0. Box 321

Richland, WA 99352

C. R. Abraham

4

Defense Nuclear Facilities Safety Board

625 Indiana Ave, N. W., Suite 700

Washington, D. C. 20004

Dan Burnfield

Lester Clemons Suite 700

Paul Gubanc Suite 700

Dermot M. Winters, Geological Engineer

2

Westinghouse Idaho Nuclear Corporation

P. 0. Box 4000

Idaho Falls, ID 83403-4000

B. Griebenow

MS -5104

A. P. Hoskins

MS -5217

1

C. Abrams

1987 Virginia Drive

Idaho Falls, ID 83404

1

F. Carlson

6965 North, 5th West

Idaho Falls, ID 83401

Distr-5 
WHC-EP-0182-58

Distribution - continued

Joseph J. DiNunno, Engineering Consultant

44 Carriage Lane

Annapolis, MD 21401

1

D. Oakley

409 12th Street, SW, \#310

Washington, D. C. 20024

1

Dr. A. Veletsos

Department of Civil Engineering

Rice University

P. 0. Box 1892

Houston, TX 77252

Westinghouse Materials Company of Ohio

P. 0. Box 398704

Cincinnati $\mathrm{OH}$ 45239-8704

David L. Jacoboski

Senior Engineer, Technology Demonstration

4

Westinghouse Savannah River Company

P. 0 . Box 616

Aiken, SC 29802

D. M. Barnes, 773-41A

J. R. Chandler, 703-H

P. d'Entremont

F. G. McNatt, 704-8A

Institute for Energy and Environmental Reseach 6935 Laurel Avenue

Takoma Park, MD 20912

Dr. Arjun Makhijani, President

SPAR Aerospace Ltd.

20 Avon Meadow Lane, Suite 220

Avon, CT 06001

Peter W. Kruse

Advanced Technology Systems Division

Redzone Robotics, Inc.

2425 Liberty Ave

Pittsburgh, PA 15222-4639

David W. White 
WHC-EP-0182-58

Distribution - continued

National Research Council. National Academy of Sciences 2101 Constitution Ave., N. W. Washington D. C. 20418

Robert S. Andrews, Senior Staff Officer

Board on Radioactive Waste Management

Converse Consultants

$18 \mathrm{~W}$. Mercer Street, Suite 300

Seattle, WA 98119

David Stanley

1

Brown \& Caldwell

100 W. Harrison

Seattle, WA 98119

Hal Cooper

Benton County Department of Emergency Management

P.0. Box 6144

Kennewick, WA 99336

Gary Pira

BOVAY Northwest Inc.

660 Swift, Suite D

Richland, WA 99352

T. J. Mclaughl in

Omega Environmental Technology

655 Montgomery Street, Suite 1000

San Francisco, CA 94111

Mike Bailey

GEC ALSTHOM Engineering Systems

P. 0. Box 1274

Richland, WA 99352

J. W. Riddington,

Vice President, Nuclear Marketing

1

T. S. Elleman

North Carol ina State University

Department of Nuclear Energy

P. 0. Box 7909

Raleigh, NC 27606

1

Mike Lingle

Stone \& Webster

7677 E. Berry Ave

Englewood, CA 80111

Distr-7 
WHC-EP-0182-58

\section{Distribution - continued}

Bryant Mather

Corps of Engineers

WESSV - Z

3909 Halls Ferry Rd

Vicksburg, MS 39180-6199

1

Paul Shewmon

Prof. Metallurgical Engineer

Ohio State University

2477 Lytham Road

Columbus, $\mathrm{OH} 43220$

\section{Waste Management External Advisory Committee Members}

Dr. Frank L. Parker

Professor of Environmental and Water Resources Engineering Vanderbilt University

P. 0. Box 1596, Station B

Nashville, TN 37235

Dr. Bruce R. Kowalski

Professor of Chemistry, Co-director of Center for Process

Analytical Chemistry

University of Washington

Chemistry Department, BIdg 10

Seattle, WA 98195

Dr. Greg R. Choppin

Professor of Chemistry

Floricla State University

Department of Chemistry, B-164

Tallahassee, FL 32306

Dr. Chester Grelecki

President, Chief Scientist

Hazards Research Corporation

200 Valley Road

Mt. Arlington, NJ 07856

Dr. Alfred Schneider

MIT Department of Nuclear Engineering

Room 24-1098

77 Massachusetts Avenue

Cambridge, MA 02139

Dr. Gary Powers

President

Design Science, Inc.

163 Witherow Road

Sewickley, PA 15143 
WHC-EP-0182-58

Distribution - continued

2

Ames Laboratory

7 Spedding $\mathrm{Hall}$

Iowa State University

Ames, IA 50011

Bill Haas

R. B. Thompson

1

RKK Ltd.

16404 Smokey Pt. Bivd, Suite 303

Arlington, WA 98223

Chris Reno

1

MACTEC

8320 Centerbrook Place

Alexandria, VA 22308

Stan Blacker

1

Engineering-Science, Inc.

1955 Jadwin Ave, Suite 470

Richland, WA 99352

Matt Sakach

1

EBASCO Services, Inc.

1201 Jadwin Avenue, Suite 202

Richland, WA 99352-3429

F. J. Young

1

MTL Systems, Inc.

3481 Dayton-Xenia Road

Dayton, OH 45431-0299

E. McDaniel

1

Nuclear Consulting Services, Irc. 7000 Huntley Road

P. 0. Box 29151

Columbus, $\mathrm{OH} 43229$

Dr. J. Louis Kovach

1

Battelle Laboratories

505 King Avenue

Columbus, $\mathrm{OH}$ 43201-2693

Rob Taylor Jr., P.E.

Rm 13-6-016 
WHC-EP-0182-58

\section{Distribution - continued}

1

Portland General Electric Co.

121 S. W. Salmon St.

Portland, OR 97204-2991

Wayne Lei, 3WTCBRO5

\section{OFFSITE - FOREIGN}

1

British Nuclear Fuels Ltd

Risley Warrington

Cheshire WA3 6AS

United Kingdom

Howard A. Edwards

1 Ricardo Hitec Ltd

Club street Works, Bamber Bridge

Preston, PR5 6FN

United Kingdom

P. K. J. Smith

1

Telerobot

Consorzio Telerobot

Via Hermada 6

16154 Genova, Italy

Bruno Sessarego

1

SGN

1 , rue des Herons, Montigny-C-Bretonneux

78132 Saint-Quentin-en-Yvelines Cedex,

France

Serge Merlin

2

CEA - Saclay

DCC/DIR

$B a^{\prime} t 121$

$91190 \mathrm{GIF} /$ Yvette Cedex

France

G. Baudin

R. Atabek

1

Hans Wal ischmiller GmbH

D-7778 Markdorf/Bodensee

Germany

Wolfgang Walischmiller 
WHC-EP-0182-58

Distribution - continued

ONSITE

G. E. Bishop

K. W. Bracken

G. J. Bracken

S. T. Burnum

N. R. Croskrey

R. C. Cullison

J. J. Davis

L. Erickson

R. E. Gerton

W. F. Hendrickson

R. D. Hildebrand

P. E. LaMont

B. L. Nico!l

T. Noble

R. H. Pestes

L. E. Petersen

G. W. Rosenwald

G. H. Sanders

E. J. Senat

J. B. Sullivan

A. D. Toth

W. R. Wresinski

J. K. Yerxa

Reading Room
R2-62

A5- 10

A4-02

A5-16

R1-30

A5-55

A5-16

A5-16

A4-02

A4-02

A5-55

A5-16

A5-16

A4-02

A5- 16

A5- 10

A4-02

A5- 16

R2 -62

A5- 10

R2 -62

A5- 16

A5- 15

Al -65

Kaiser Engineering Hanford

C. J. Denson

E6-51

J. E. Fasso Jr.

S3-10

D. J. Shrimpton

E6-21

Stone \& Webster Engineering Co.

E. L. Richards

R2 -83

1

MACTEC

J. Janus

G6-18

Pacific Northwest Labor atories

D. B. Baird

K7 -34

D. W. Bennett

$\mathrm{K} 5-22$

S. A. Bryan

P7 -25

L. L. Burger

P7 -25

J. F. Fletcher

K7-97

L. K. Holton Jr.

P7 -43

J. Janata

$\mathrm{K} 2-12$

B. M. Johnson

$\mathrm{K} 1-78$

E. 0 . Jones

P8-38 
WHC-EP-0182-58

\section{Distribution - continued}

$\begin{array}{ll}\text { L. G. Morgan } & \text { P7-35 } \\ \text { B. E. Opitz } & \text { K6-81 } \\ \text { M. S. Peffers } & \text { K7-94 } \\ \text { W. G. Richmond } & \text { P7-41 } \\ \text { R. D. Scheele } & \text { P7-25 } \\ \text { P. A. Scott } & \text { P7-19 } \\ \text { J. C. Spanner } & \text { K2-05 } \\ \text { D. Strachan } & \text { K2-38 } \\ \text { K. L. Steinmaus } & \text { K6-84 } \\ \text { R. S. Wegeng } & \text { K7-97 } \\ \text { P. D. Whitney } & \text { K7-34 } \\ \text { T. W. Wood } & \text { K6-47 }\end{array}$

Westinghouse Hanford Company
A. T. Alstad
R1-49
R. P. Anantatmula
R2-11
J. D. Anderson
N3-11
J. N. Appel
I. J. Austin
H. Babad
S4-58
T4-01
A. D. Bates
$\mathrm{R} 2-78$
T6-07
P. K. Bhatia
S4 -58
T. J. Bander
$\mathrm{HO}-33$
L. L. Barry
R1 -67
G. D. Bazinet
D. L. Becker
L4-71
H5-57
D. B. Bechtold
T6-09
K. H. Bergsman
L6-24
M. V. Berriochoa
B3 -30
D. L. Bjorklund
S6-01
J. E. Bjorklund
R1-62
R. J. Bl anchard
$\mathrm{R} 1-17$
D. C. Board
S1 -57
K. D. Boomer
H5-49
G. L. Borsheim
R2-11
V. C. Boyles
R1 -49
H. R. Brager
L5-65
D. R. Bratzel
L5-31
R. G. Brown
R2 - 14
J. G. Burk, Jr.
B3 -25
J. H. Bussell
L7 -06
J. A. Caggiano Jr
H6-06
K. G. Carothers
$\mathrm{R} 1-51$
J. W. Carey
SO-01
R. J. Cash
R2 - 32
G. Christensen
$\mathrm{H} 4-21$
H4 -61
H6- 07
F. M. Coony
H4 -61
W. L. Cowley
Rl -30
N. R. Croskrey
R1 - 51
G. M. Crummel
L5-63
J. M. Cruse
SO-02 
L. T. Cunningham

J. I. Dearing

C. DeFigh-Price

T. A. Demitruk

D. R. Dickinson

E. C. Ladd

R. A. Dodd

M. R. Duncan

G. L. Dunford

W. S. Dunnivant

J. A. Eacker

D. R. Ellingson

M. F. Erhart

S. D. Estey

W. G. Farley

K. 0. Fein

R. A. Flores

L. A. Fort

K. D. Fowler

G. L. Fox, Jr.

G. T. Frater

J. R. Freeman-Pollard

J. C. Fulton

K. A. Gasper

G. J. Gauck

R. L. Gilchrist

D. A. Gilles

S. D. Godfrey

D. J. Green

P. R. Golberg

J. M. Grigsby

V. W. Hall

C. S. Haller

D. G. Hamrick

K. L. Hampsten

B. M. Hanion (30)

J. M. Hanson

H. D. Harmon

J. P. Harris III

J. M. Henderson

D. W. Hendrickson

N. A. Hertelendy

E. G. Hess

ii. C. Higginson

J. G. Hill

B. M. Hisaw

J. D. Hopkins

B. K. Horsager

R. D. House

J. H. Huber

J. L. Huckaby

L. L. Humphreys

J. E. Hysjulien

J. E. Irwin
T5-55

H5-72

R2-31

H5 - 36

L5-31

R1-19

R1-51

L6-10

R1-51

R2-88

R2-50

H5-37

R1-51

R2-11

H4 -62

H4-63

SO-12

S4-57

R2-11

L5-01

R1-51

H6-03

R2-31

R2-08

R1-51

L5-63

S2-14

R1-51

H5-53

B4-08

H4-62

B1-59

G6-04

R1-51

L4-71

R1-80

B1-58

R2-52

S4-55

S4 - 55

R4-03

H5-09

R3-09

A4-25

R2 - 12

R1 -62

R2-11

S4 -57

R2-83

R1 -49

R2-11.

R2 - 50

SO-09

B1-59 
M. N. Islam

G. D. Johnson

J. L. Juette

L. J. Julyk

R. A. Kirkbride

N. W. Kirch

W. L. Knecht

E. M. Koellermeier

G. M. Koreski

A. G. Krasopoulos

M. Kummerer

M. J. Kupfer

D. R. Lance

J. E. Langdon

D. L. Lenseigne

D. C. Lini

P. J. Mackey

G. T. MacLean

M. K. Mahaffey

R. M. Marusich

V. D. Maupin

T. B. McCall

J. D. McCormack

K. S. McCullough

M. E. McDonald

M. A. McLaughl in

T. E. Mensinger

N. J. Milliken

W. J. Millsap

G. J. Miskho

J. R. Mobley

K. L. Morris

J. P. Mullally

L. D. Muhlestein

A. F. Noonan

T. W. Oden

P. C. Ohl

D. B. Pabst

R. B. Pan

I. G. Papp

G. L. Parsons

M. A. Payne

T. B. Powers

R. K. P'Pool

J. G. Propson

T. E. Rainey

R. E. Raymond

R. W. Reed

I. E. Reep

M. A. Rezvani

D. Richardson

J. H. Roecker

L. Ruffin

J. A. Ryan
R3-08

R2-78

G6-56

H5-56

S4-58

R2-11

HO-34

R4-40

R1-51

A5-55

H4 -62

H5- 49

SO-09

S2-24

R2 -75

H5- 49

B3-15

S4 -58

L4 - 73

H4 -60

R1-51

HO -33

L5-31

H5-34

$\times 3-68$

H5- 09

T3-10

H4 -62

H5-68

R2 - 50

R2 - 18

H5- 09

S4-57

N1 - 28

R2 - 12

R2 -18

H5- 09

B2-35

H5 -53

R3-45

S4-57

R2-50

H4 -65

T1-30

R2-18

Rl -49

R1 -80

R1-51

R2-08

H5-55

R2-31

B1-59

R1-51

H5- 55 


\section{Distribution - continued}

\begin{tabular}{|c|c|}
\hline & \\
\hline $\begin{array}{l}\text { C. C. Scaiei } \\
\text { F. A. Schmorde } \\
\text { J. S. Schofield } \\
\text { C. P. Schroeder } \\
\text { D. D. Scott } \\
\text { K. V. Scott } \\
\text { R. A. Shea } \\
\text { J. E. Shapley } \\
\text { T. N. Shaw } \\
\text { E. M. Sheen } \\
\text { E. J. Shen } \\
\text { P. K. Shen } \\
\text { A. T. Shook } \\
\text { A. B. Sidpara } \\
\text { C. M. Smith } \\
\text { S. G. Spencer } \\
\text { S. M. Stahl } \\
\text { E. G. Stephan } \\
\text { R. R. Stickney } \\
\text { T. L. Strathman } \\
\text { J. N. Strode } \\
\text { K. C. Strong } \\
\text { M. J. Sutey } \\
\text { D. G. Sutherland } \\
\text { J. P. Summerhays } \\
\text { L. M. Swanson } \\
\text { J. F. Thompson } \\
\text { J. D. Thomson } \\
\text { S. R. Tifft } \\
\text { J. A. Tilden (2) } \\
\text { H. Toffer } \\
\text { T. T. Tran } \\
\text { J. B. Truitt } \\
\text { C. J. Udell } \\
\text { R. E. Vandercook } \\
\text { R. J. Van Vleet } \\
\text { J. A. Voogd } \\
\text { J. R. Weber } \\
\text { D. L. Wegener } \\
\text { R. K. Welty } \\
\text { G. T. Wells } \\
\text { K. A. White } \\
\text { D. D. Wodrich } \\
\text { B. D. Zimmerman } \\
\text { Docket File (2) } \\
\text { 272-AW Shift Office } \\
\text { Central Files (2) } \\
\text { Tank Farms Info Center } \\
\text { Information Release } \\
\text { Administration (3) } \\
\text { Env Data Mgmt Center }\end{array}$ & \\
\hline
\end{tabular}



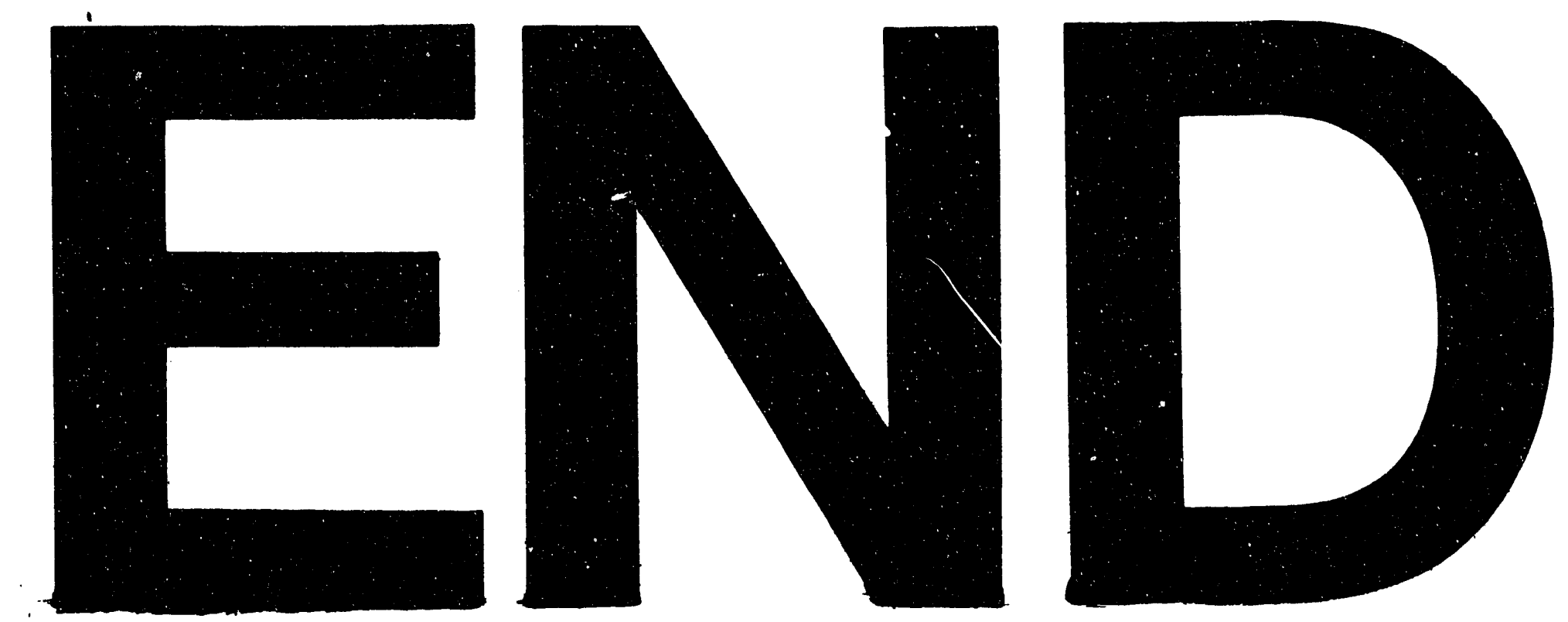

II
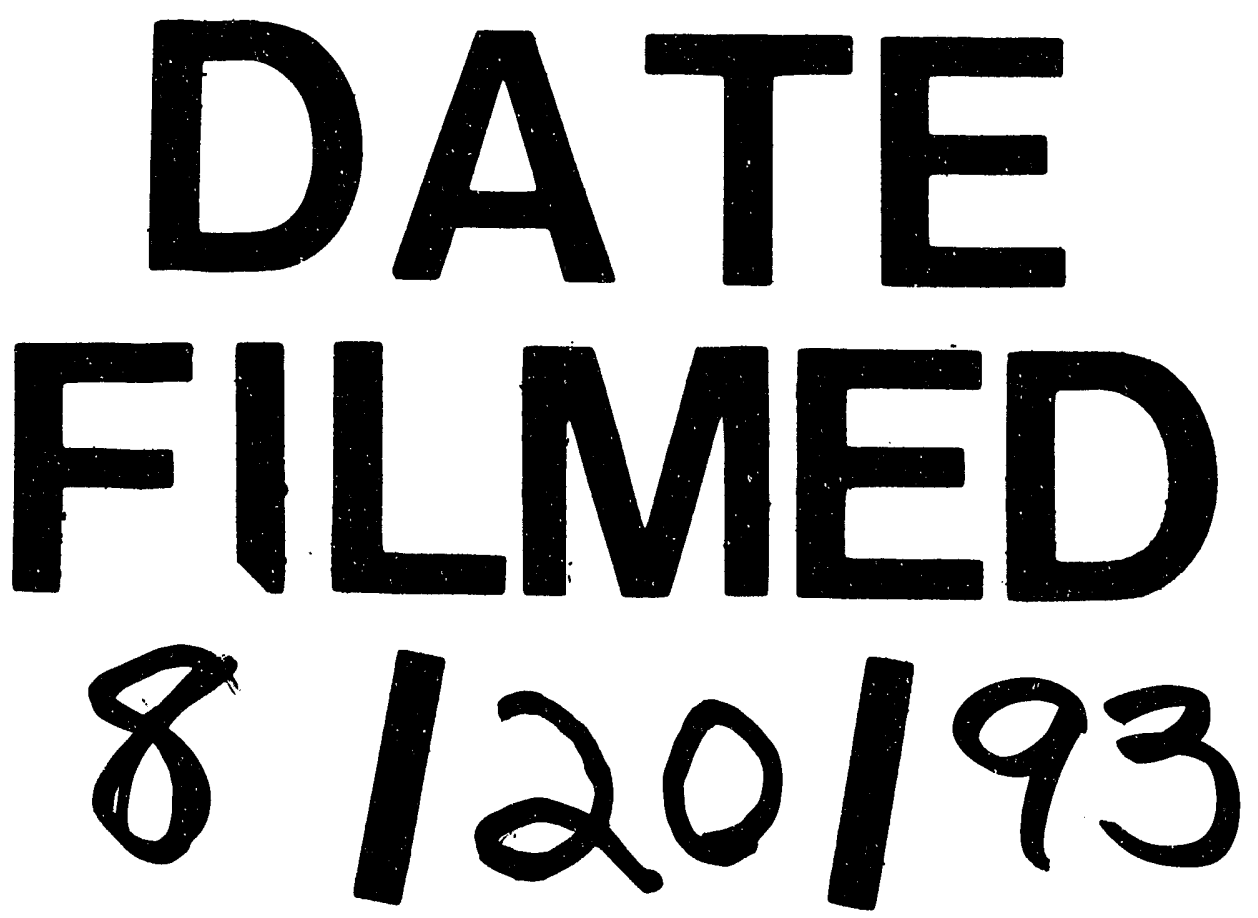
